$$
\text { DOE/CHIO3000-T25 }
$$

\title{
A STUDY OF THE ABILITY OF THE DO DETECTOR TO MEASURE THE SINGLE JET INCLUSIVE CROSS SECTION
}

By

\section{0 \\ c66) 91190 \\ ดヨ^1コวコบ}

Richard Vincent Astur

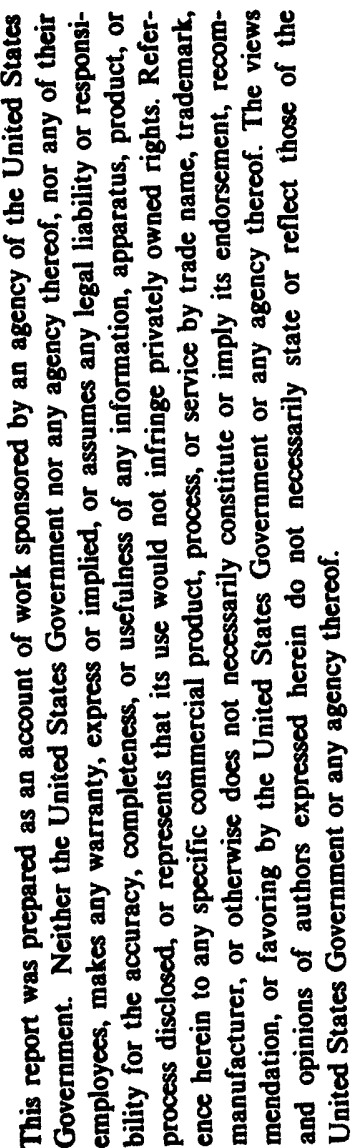

\author{
A DISSERTATION \\ Submitted to \\ Michigan State University \\ in partial fulfillment of the requirements \\ for the degree of
}

DOCTOR OF PHILOSOPHY

Department of Physics and Astonomy

1992

ACO2-76CHO30OO 


\title{
ABSTRACT A STUDY OF THE ABILITY OF THE DO DETECTOR TO MEASURE
THE SINGLE JET INCLUSIVE CROSS SECTION
}

\author{
By
}

\section{Richard Vincent Astur}

The D0 experiment began accumulating data at the Fermilab Tevatron in May of 1992. Protons are collided with antiprotons at $\sqrt{s}=1.8 \mathrm{TeV}$ and an expected peak luminosity of $5 \times 10^{30} \mathrm{~cm}^{-2} \mathrm{sec}^{-1}$. The D0 detector is an all-purpose detector that will have exceptional jet reconstruction capabilities derived from superior calorimetry and nearly $4 \pi$ angular coverage. One of the many physics measurements that will be made at $\mathrm{D} 0$ is the inclusive jet cross section. Comparison of this cross section with theoretical predictions allows us to test the accuracy of the standard model of Quantum Chromodynamics (QCD). This comparison is usually in the form of a measurement of the differential cross section with respect to the transverse energy of the jet. The extended angular coverage of the D0 detector allows measurements of the differential cross section as a function of jet rapidity as well. Recently completed calculations of the next-to-leading-order contribution to the inclusive cross section result in predictions with reduced theoretical errors. In addition, recent fits to data from deep inelastic scattering and single photon experiments further restrict the quark and gluon structure functions of the proton which are necessary in the theoretical predictions of the cross section. It may be that an experimental measurement of the inclusive cross section would favor some of these fits over others. We have studied extensive computer simulations of both the Tevatron and the D0 detector in order to determine D0's ability to reconstruct the inclusive cross section. We present a discussion of this analysis as presently understood. 
To my parents, Oscar Antonio and Maryjane Astur, for all they have given me. 


\section{ACKNOWLEDGEMENTS}

There are an almost uncountable number of people without whose time and effort in both large and small ways would have made this work impossible or at least much moie difficult and less enjoyable than it was. In particular I would like to thank the following people: Barbara Taylor, Stephanie Holland, Teresa Thomas and Dolores Foote for taking care of all my administrative worries at Michigan State both while I was there and when I was away; Paul Russo, Kurt Ruthmansdorfer and John Featherly for superb computer support at D0 and FNALD0; Nick Hadley, Boaz Klima, Harrison Prosper, Chip Stewart and the rest of the D0 software support group for all their work developing, debugging and testing D0 parton jet and jet reconstruction software; all the work and contributions of the D0 QCD group; Jan Guida and Joan Guida to whom I owe a great deal of my calorimeter and DAQ knowledge, Alan Jonckheere who answered every dumb question I ever asked with detailed e-mail instructions; Dan Edmunds and Philippe Laurens for all their expertise in regards to the Level 1 Calorimeter trigger and simulator and Jan Hoftun for helping me understand the Level 2 filiering system. I would like to thank my professors at Michigan State and all the students, physicists and staff who have worked on the D0 experiment. Most of all, I would like to thank my advisor, Harry Weerts, for his guidance and advice over the last six years (even if I didn't always take it, I appreciate it just the same).

Richard Astur May 10, 1992 


\section{Table of Contents}

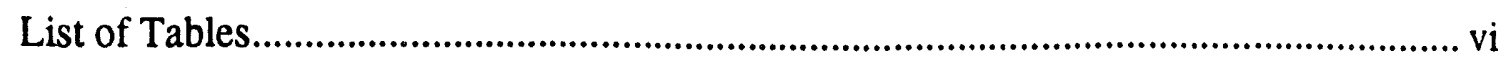

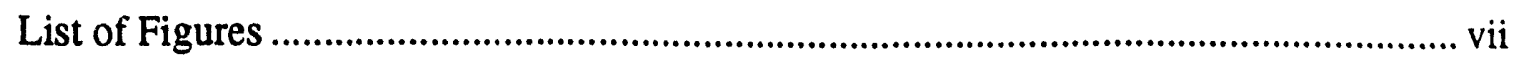

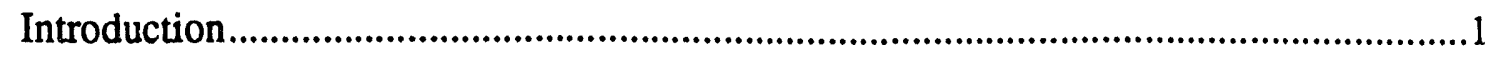

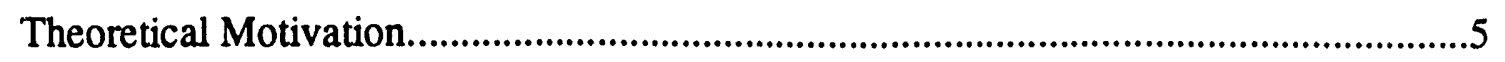

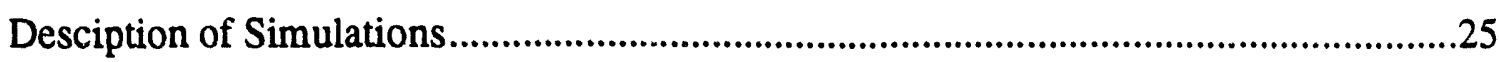

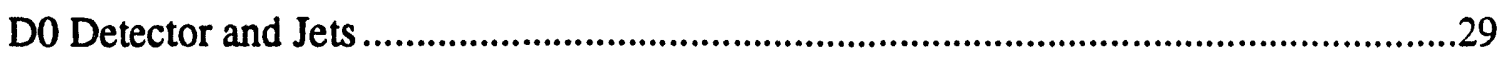

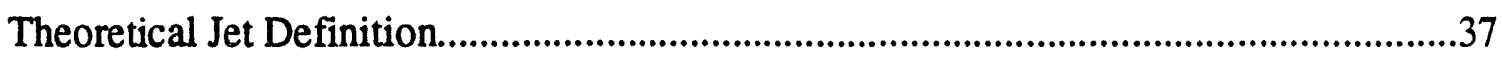

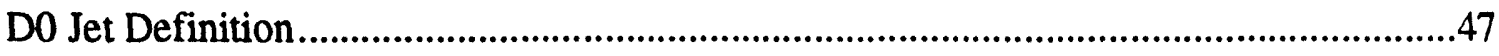

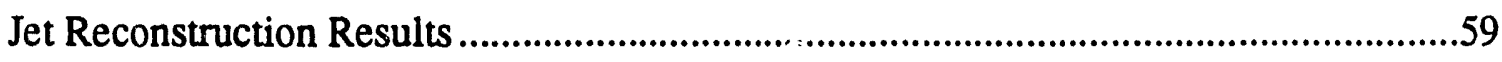

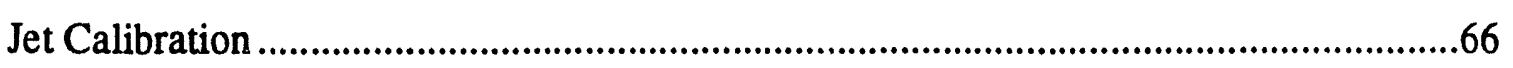

Jet Triggering at D0..........................................................................................95

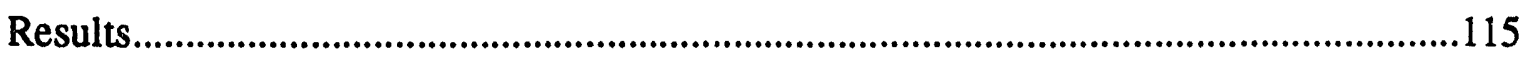

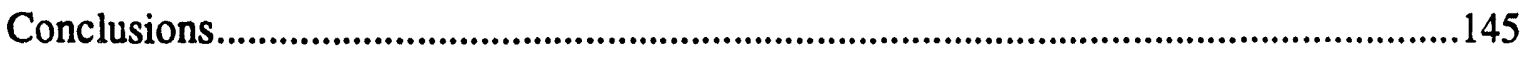

Appendix A-Level 1 Trigger Sensitivity.......................................................................147 


\section{List of Tables}

$2-1$

$2-2$

Quark Colors .6

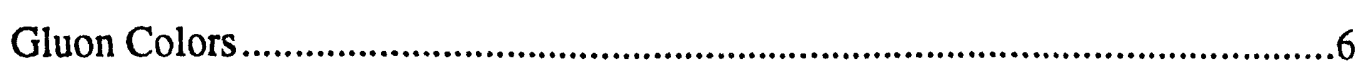

9-1

$9-2$

Proposed Level 1 Jet Triggers and Rates.......................................................106

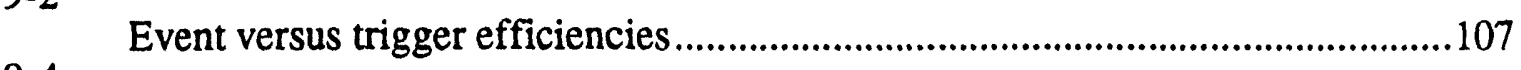

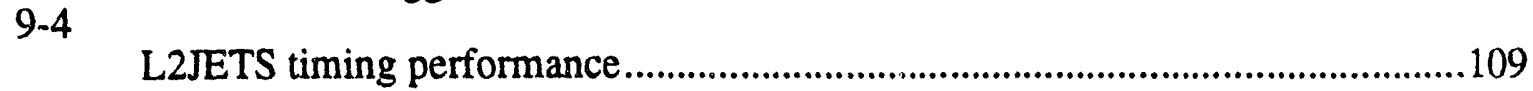

9-5

$9-3$

L2JETS jet reconstruction compared to offline reconstruction 109

Proposed Level 1 and Level 2 jet triggers and rates ......................................110

$10-2$

AA-1

Parameter Values for Various Parton Distribution Fits .....................................135

AA-2

Jet Et distribution for Herwig and Isajet. ......................................................148

AA-3

Ratio of Level 1 Trigger Tower efficiency with noise to without .....................150

Ratio of Trigger Efficiency with Old Smearing Compared to New

Smearing. 151 


\section{List of Figures}

2-3

Gluon Couplings ................................................6

$2-4$

Quark Structure of Hadrons........................................... 7

$2-5$

Hard Scattering of Partons .........................................8

$2-6$

Example of a Leading Order Process .............................. 9

$2-7$

$2-8$

Example of a Next-To-Leading-Order process ..................... 10

$2-9$

Example of a higher order process ............................... 10

$2-10$

Typical Proton Parton Distribution Functions

Cross section Dependence on $\mu^{2}$ for LO and NLO ................... 15

$2-11$

NLO Inclusive Jet Cross Section versus ET

$2-12$

Dependence of Theoretical ET Distribution on $\mu^{2} \ldots \ldots \ldots \ldots \ldots \ldots \ldots \ldots . \ldots 18$

$2-13$

NLO Inclusive Jet Cross Section versus Rapidity.

$2-14$

Example of Quark Fragmentation

4-1

4-2

Central Detector, Central Calorimeter and Level 0 Scintillators. .31

D0 Calorimeters 33

4-3

Calorimeter Readout Cell 35

$5-1$

5-3

Theoretical cone definition for jets.

Cross Sectional Dependence on the Cone Size $\mathbf{R}$ 41

Jet ET Distributions for Various PJET Algorithms. 44

$6-1$

$6-2$

ISAJET Jet Width for Partons and Particle Jets 49

$6-3$

Typical T'wo Jet Event as seen by the DO Detector .52

Number of Ellis Jets 54 
viii

$6-4$

Number of Ellis Jets - Number of Reconstructed Jets

SPLF $=.50$

$6-5$

Number of Ellis Jets - Number of Reconstructed Jets

SPLF $=.75$ 56

$7-1$

Reconstructed Jet $\phi$ and $\eta$ compared to Generated

$7-2$

Reconstructed Jet E and ET Compared to Generated

$7-3$

Jet Reconstruction Efficiency

$8-1$

$e / \pi$ as a function of particle energy and detector $\eta$

$R(E)$ and $R(E T)$ for single electrons

$R(E)$ and $R\left(E_{T}\right)$ for single pions

Fractional Energy Carried by Particles within an Average Jet 70

Fractional Energy Carried by Particles with $<5 \%$ of the Jet's

Pion Response with and without a Cone 73

8-6

$R(E)$ versus $\eta$ for Different Cone Sizes 74

$\eta$ bias due to particle showering. 75

$R(E)$ for Uncalibrated Jets. 79

$R(E)$ for Jets Calibrated Using Particles within the Jet 80

R(E) for Jets Calibrated Assuming all Particles are Pions

$R(E)$ for Jets Calibrated using Slope of the Pion Response

$8-11$

Curve

Example of Particle Tracking Method for Jet Calibration. 83

$8-13$

$R(E)$ for Jets Calibrated with Track Cluster Method (leading particle)

$8-14$

$R(E)$ for Jets Calibrated with Track Cluster Method (share) 86

$8-15$

$R(E)$ versus Jet Characteristics 88

8-16

$8-17$

$R(E)$ for jets made with various generators ......................... 89

Predicted Fractional Energy Loss for Various Event Generators. 91 
ix

8-18

$8-19$

$R(E)$ for Uncalibrated Jets Compared with the Predicted Values........ 92

9-1

$R(E t)$ for Uncalibrated Jets Compared with the Predicted Values

9-2

D0 Trigger and Data Acquisition System

The $\eta$ segmentation of the calorimeters in terms of Trigger

Towers.

$9-4$

Trigger Efficiency versus Jet ET for one Level 1 trigger setting

$9-3$

Ratio of Level 1 Trigger Quantities to the Jet ET

$9-5$

Trigger Efficiency versus Jet $\mathrm{E}_{\mathrm{T}}$ for Various Level 1 Trigger

Settings

$9-6$

Level 1 Rate and Efficiency versus trigger tower threshold

$9-7$

Jet Trigger Rate and Efficiency versus L2JET ET Threshold

$9-8$

Ratio of L2JET Jet ET to Reconstructed Value

$10-1$

Correction of Reconstruction Algorithm with Incorrect Splitting

$10-2$

Fraction

Predicted and Real R(ET)'s for Two Cone Sizes......................... 120

10-3

$R(E)$ With and Without $0.1 \mathrm{GeV}$ Zero Suppression

$10-4$

$\mathrm{R}(\mathrm{ET})$ for both Real and Adjusted Predicted

$10-5$

$10-6$

Ratio of Uncalibrated to Generated Cross Section

Ratio of Predicted Calibrated to Generated Cross Section.

$10-7$

$10-8$

Ratio of Real Calibrated to Generated Cross Section

Ratio of Predicted Calibrated to Smeared Generated Cross

Section

$10-9$

$10-10$

Ratio of Real Calibrated to Smeared Generated Cross Section

Ratio of Unsmeared Predicted Calibrated to Generated Cross

$10-11$

10-17

Section.

Ratio of Unsmeared Real Calibrated to Generated Cross Section

10-12

Sensitivity of Reconstruction to a 2 percent Calibration Error 134

Jet ET Cross Section at $\eta=0$. for Various Structure Functions 
$10-13$

$10-14$

Jet ET Cross Section at $\eta=2$. for Various Structure Functions .......... 138 Jet $\eta$ Cross Section at ET=10. for Various Structure Functions............ 139

$10-15$

Jet $\eta$ Cross Section at ET=60. for Various Structure Functions............ 140

$10-16$

10-18

Cross Section Dependence on Parton Distribution-LO vs NLO ........... 142

P.D.F. Differences Compared with Reconstruction ................... 143 


\section{Chapter 1: Introduction}

Hadrons, like the proton, are believed to be composed of more elementary particles called partons. Partons have been grouped into two categories: charged fermions called quarks and the neutral bosons known as gluons. Evidence for the existence of partons came initially from jets seen at electron-positron colliders while the parton structure of the proton was inferred more directly from Deep Inelastic Scattering (DIS) of neutrinos and electrons from protons. Collisions at greater energies were studied at proton-proton and then proton-antiproton colliders ${ }^{1}$. Currently, the Tevatron at the Fermi National Accelerator Laboratory in Batavia, Illinois collides protons with antiprotons at a center-of-mass energy of $1.8 \mathrm{TeV}$, the highest in the world. In all cases, the scattering and liberation of a parton were evidenced by sprays of particles at wide angles to the beam line after a collision. These sprays of particles are referred to as jets and are widely accepted as being representative of a scattered parton of approximately the same direction and energy.

At the Fermilab Tevatron, jet production is one important way to experimentally test Quantum Chromodynamics (QCD), the theory that describes the interactions of quarks and gluons. The simplest experimental jet measurement one could make would be the cross section for $\mathrm{p} \overline{\mathrm{p}} \rightarrow \mathrm{jet}+\mathrm{X}$. Here $\mathrm{X}$ denotes any other product of the interaction. This is known as the single jet inclusive cross section for proton-antiproton interactions. This can be compared to the $Q C D$ predictions for $p \bar{p} \rightarrow$ parton $+X$ in those kinematic regions where $Q C D$ is able to make such predictions.

QCD predictions can currently only be calculated for those collisions in which there is a 'large' momentum transfer between the colliding hadrons. In these cases, a parton is exchanged which is sufficiently energetic to resolve the partons within the 
hadron. 'Large' is determined by the relation of the momentum transfer, $Q$, to a $Q C D$ parameter, $\Lambda$, which must be measured. In the kinematic region where $Q^{2}>\Lambda^{2}$, the proton-antiproton interaction is expected to act like independent elastic scattering between individual partons because the strong coupling constant, $\alpha_{\mathrm{S}}$, asymptotically approaches zero as $\mathrm{Q}$ is increased without bound. For small $\alpha_{\mathbf{S}}$, calculations can be performed perturbatively in $\alpha_{\mathrm{s}}$. These calculations quickly become complicated and time consuming as the order in $\alpha_{S}$ is increased, however. For this reason, many measurements of the inclusive cross section have been compared only to the leading order (LO) $\alpha_{s}{ }^{2}$ predictions. Recent experiments ${ }^{2}$ have had the benefit of predictions for next-to-leading-order (NLO) $\alpha_{\mathrm{s}}{ }^{3}$ jet production at proton-antiproton colliders ${ }^{3}$. Theoretical errors in the prediction are greatly reduced in the NLO calculation. ${ }^{4}$ However, there is still a $15-20 \%$ uncertainty which results from imperfect knowledge of the momentum distributions of the quarks and gluons within the proton. Nominally the proton consists of 2 valence up quarks and a valence down quark. But there is also a large gluon presence as well as a lesser occurrence of other quark flavors. Further, none of the parton types carries a fixed fraction of the proton's momentum but rather have some distribution as a function of that fraction. These are described by the proton parton distribution function (p.d.f.) for each parton. Being the largest source of experimental errors, it may be possible that comparison of predictions for various sets of parton distribution functions to a precision experimental measurement could prefer some sets over others 5 . Further errors in using QCD theory to predict the jet cross section are associated with the definition of a jet and the assumption that the scattered parton kinematics can be inferred from measurements made on the jet. We will discuss jet definitions in detail in later chapters.

The $\mathrm{D} 0$ detector $^{6}$ is an all-purpose detector that is especially suited to jet detection and reconstruction. It consists of a central detector which identifies charged tracks and the location of the collision, a hermetic calorimeter for containing and measuring the energy of all particles that are produced (except neutrinos and muons) and a muon system that detects and reconstructs muons. Neutrinos are detected indirectly through the measurement of transverse momentum imbalances in the detector. D0 has begun taking data in the spring of 1992 at the Fermilab Tevatron where protons and antiprotons collide with a center of mass energy of $1.8 \mathrm{TeV}$ and an expected peak luminosity of $5 \times 10^{30} \mathrm{~cm}^{-2} \mathrm{sec}^{-1}$. Various elements of the DO detector have been subjected to the Fermilab fixed energy particle beam (DO Test beam) for calibration and study ${ }^{7}$. The detector has also undergone numerous commissioning runs using cosmic ray muons to test both detector and data acquisition performance. ${ }^{8}$ Additionally, the 
performance of the D0 detector at the Fermilab Tevatron has been simulated extensively. To simulate proton-antiproton collisions, event generators such as ISAJET ${ }^{9}$, HERWIG ${ }^{10}$ and PYTHIA $^{11}$ were used. These programs vary in how they simulate $Q C D$ above leading order and how they fragment the resulting partons into final state particles. The response of the $\mathrm{DO}$ detector to these particles is simulated by combining the GEANT ${ }^{12}$ physics package with details of D0 geometry to form a program named DOGEANT. The GEANT portion simulates various physics processes that can cause leptons and hadrons in the detector to scatter, shower and deposit energy.

In the succeeding chapters we will discuss DO's ability to measure the single jet inclusive cross section based on these simulations. We will start with a detailed description of the simulated data that was analyzed as well as further theoretical motivation behind the jet cross section. The theoretical and D0 experimental definitions of jets will be discussed. A brief overview of the DO detector and its role specifically in reconstructing and detecting jets will be presented. Predictions for jet reconstruction and detection will be discussed as well as methods for correcting biases in this reconstruction. Finally, we interpret the results in terms of the predicted accuracy of reconstructing the single jet inclusive cross section at $\mathrm{D} 0$ and make conclusions based on the entire analysis. 
'See numerous papers by the CDF, UA1 and UA2 collaborations.

${ }^{2}$ CDF Collaboration, Jet Results from CDF pubiished in the Proceedings XXVth Reconstres de Moriond, (1991)

${ }^{3}$ S. D. Ellis, Z. Kunszt and D. E. Soper, Phys. Rev. Lett. 642121 (1990)

${ }^{4}$ Ibid Ellis. (1990)

5A. D. Martin, R. G. Roberts and W. J. Stirling, Preprint RAL-90-084 (1990).

${ }^{6} \mathrm{D} 0$ Collaboration, An Experiment at DO to Study Antiproton-Proton Colllisions at 2 TeV: Design Report, Print-84-0306 (Fermilab) (1983)

${ }^{7}$ See for example M. Abolins et al., Nucl. Instr. Meth. A280 36 (1989) and H. Aihara, Performance of the DO End Calorimeter Electronic Module, LBL-29784, October 1990. and N. Amos, Proceedings of the First International Conference on Calorimetry in High Energy Physics (ed. D. Anderson, M. Derrick, H. E. Fisk, A. Para, C. Sazama), 84 (1990)

8J. Kotcher, Ph.D. thesis, New York University

9 ISAJET Event generator written by F. E. Paige and S. D. Protopopescu, Brookhaven National Laboratory

${ }^{10}$ G. Marchesini and B. Webber, Nucl. Phys., B310 461, (1988)

${ }^{11}$ Event generator written by H-U Bengtsson, Department of Physics, University of Lund, Lund, Sweden

12R. Brun et al., GEANT3 Users Guide, CERN DD/EE/84 


\section{Chapter 2: Theoretical Motivation}

As our interest in measuring the inclusive jet cross section is motivated by a desire to test the predictions of standard QCD theory, we will first discuss the relation between the two'. QCD is the theory that describes the strong force, which is credited with holding both nucleons and atomic nuclei together. In analogy with Quantum Electrodynamics (QED), QCD predicts a strong 'charge', a strong coupling constant $\left(\alpha_{s}\right)$, and exchange particles that mediate the strong force. The only particles that interact strongly are quarks and gluons. Since nucleons, like the proton, are believed to be composed of such particles, quarks and gluons are sometimes generically referred to as partons. Similarly, antinucleons are composed of antiquarks and gluons. Another way of describing QCD is then, the theory of the interactions of quarks and gluons.

\section{Strong Charge}

The strong 'charge' is called color. Quarks (antiquarks) each come in three strongly charged varieties: red, blue and green ( antired, antiblue and antigreen). Further, there are 5 experimentally detected 'flavors' of quarks and a predicted sixth flavor known as the top quark. Table 2-1 lists the quark flavors and their color charge as well as their electric charge. Gluons, unlike the analogous photons in QED, also carry strong charge. The eight colored varieties of the gluon are listed in table 2-2. 


\begin{tabular}{|l|l|l|}
\hline Quark & Charge & Color Charge Types \\
\hline Up & $+2 / 3$ & r, b or $g$ \\
Down & $-1 / 3$ & r, b or $\mathrm{g}$ \\
Strange & $-1 / 3$ & r, b or $\mathrm{g}$ \\
Charm & $+2 / 3$ & r, b or $\mathrm{g}$ \\
Bottom & $-1 / 3$ & r, b or $\mathrm{g}$ \\
Top & $+2 / 3$ & r, b or g \\
\hline
\end{tabular}

Table 2-1: Quark colors

\begin{tabular}{|l|l|l|}
\hline Gluon & Charge & Color Charge Types \\
\hline Gluon & Neutral & $\begin{array}{l}r \overline{\mathrm{b}}, \mathrm{r} \overline{\mathrm{g}}, \mathrm{b} \overline{\mathrm{g}}, \mathrm{b} \overline{r_{1}} \mathrm{~g} \overline{\mathrm{r}_{1}} \\
(\mathrm{r} \overline{\mathrm{r}}-\mathrm{b} \overline{\mathrm{g}}) / \sqrt{2}, \mathrm{~g} \overline{\mathrm{r}} \\
\text { or }(\overline{\mathrm{r}} \overline{\mathrm{r}}+\mathrm{b} \overline{\mathrm{g}}-2 \mathrm{~g} \overline{\mathrm{g}}) / \sqrt{6}\end{array}$ \\
\hline
\end{tabular}

\section{Table 2-2: Gluon colors}

The charge of the gluon allows its emission or absorption by a quark to cause the quark to change color. For example, a blue quark that absorbs a g $\bar{\sigma}$ gluon would become a green quark of the same flavor. The charge of the gluon leads to more interesting differences between QCD and QED. The charge of the gluon allows it to interact with itself as well as quarks as shown in figure 2-3.
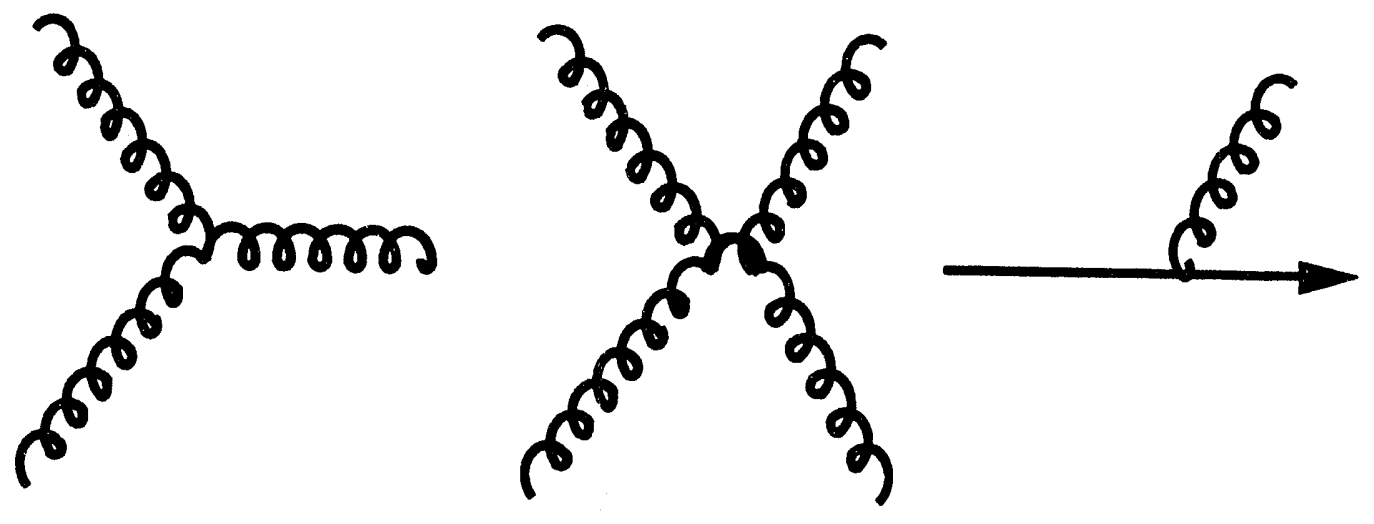

Figure 2-3: Gluon couplings. In addition to coupling with quarks, the gluon is able to couple with other gluons in several ways. 
These extra gluon interactions lead to a strong coupling constant which decreases with increasing energy of the mediating gluon as shown in equation 2-1. Strong coupling at low energies means that quarks are constantly exchanging gluons with neighboring quarks resulting in the tight bonding of quarks into colorless configurations of either 3 quarks (or antiquarks) or a quark-antiquark pair. The former is known as a baryon and the latter is known as a meson. Figure 2-4 shows the simple quark structure of both. Not shown is the exchanged gluons and quark-antiquark pairs which can be formed momentarily by the gluons.
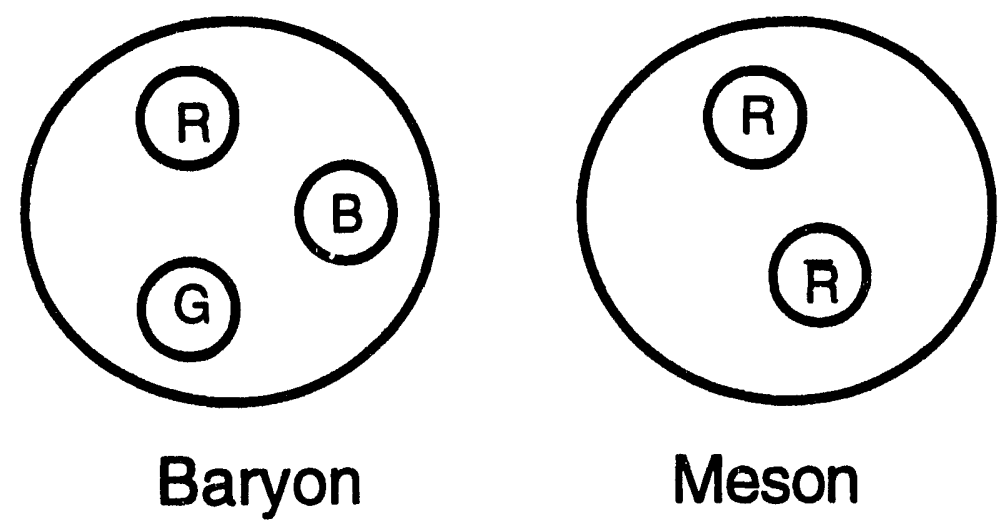

Figure 2-4: Quark structure of Hadrons. All hadrons come in two types. Baryons contain three quarks each with distinct color (antibaryons contain three antiquarks with distinct anticolor.) Mesons contain a quark and an antiquark of the same color.

\section{Strong Coupling Constant and Perturbative QCD}

The strong interactions between quarks and gluons at low energy make attempts at calculations nearly impossible. However, at the collider, where protons and antiprotons are collided at high energies, large momentum transfers between partons (mediated by energetic gluons), have decreasingly small couplings. Thus the scattered parton behaves more and more like a free particle with increasing gluon energy. This is known as the asymptotic freedom of the partons. The strong coupling constant has the form:

$$
\alpha_{s}=\frac{12 \pi}{(33-2 f) \ln \left(Q^{2} / \Lambda^{2}\right)}
$$

Here $Q$ is the 4-momentum magnitude of the exchanged gluon. $f$ is the number of quark flavors assumed to contribute. Equation 2.1 is valid only for large $Q / \Lambda$, where interactions can be calculated perturbatively in terms of the $\alpha_{s}$. Figure 2-5 
shows a high energy interaction between a proton and an antiproton in which a quark from each scatter elastically while the remaining 'spectator' partons continue on their way. Each coupling with the gluon

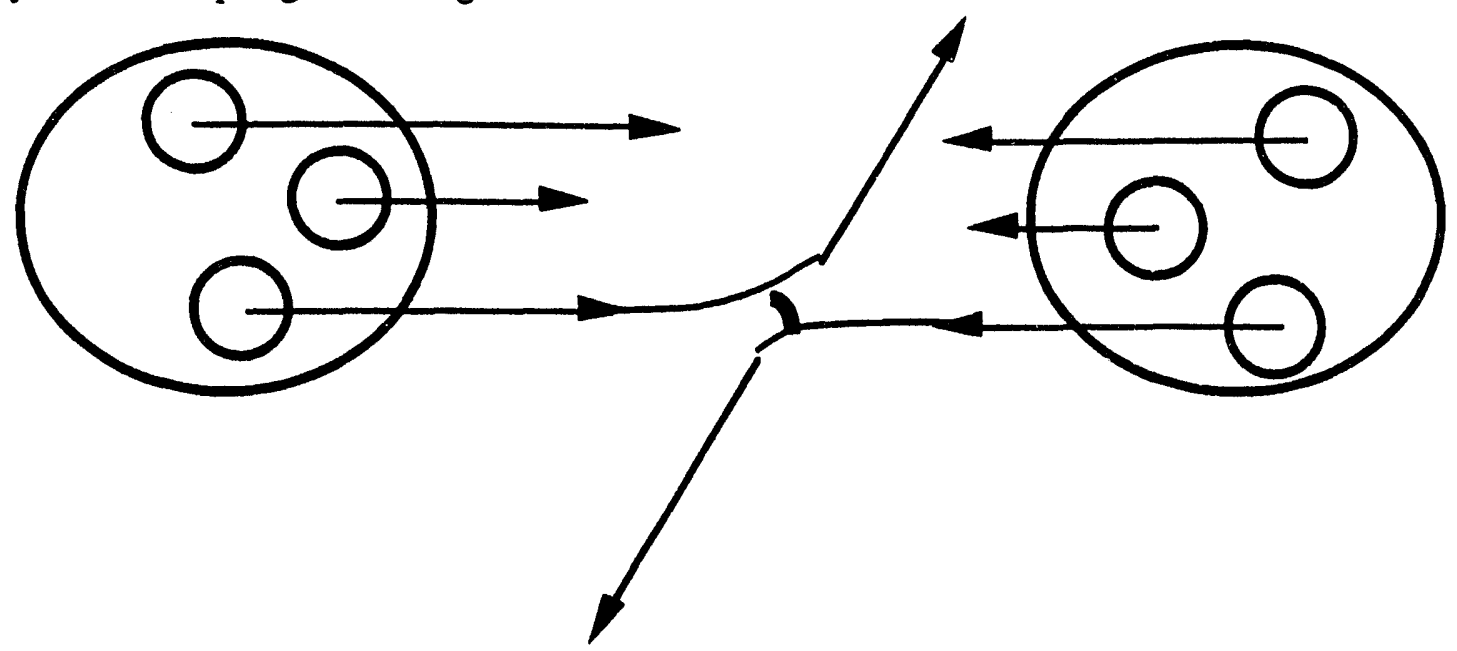

Figure 2-5. Hard scattering of partons during a proton antiproton collision. The remaining partons continue along the beamline. Two jets are formed from the scattered partons and two jets, from the remains of the baryons, continue along the beamline.

contributes a $\sqrt{\alpha_{S}}$ to the amplitude and the lowest order calculation that could be made would involve two partons scattering to two partons in the final state. Figure 2-6 shows an example of such $2 \rightarrow 2$ interactions. The cross section for such a calculation would be proportional to $\alpha_{s}{ }^{2}$ which we refer to as leading order (LO). The next-toleading-order (NLO) calculation would involve, additionally, terms of $\alpha_{s}{ }^{3}$. Some of these terms involve the emission of an energetic gluon away from a parent parton as shown in figure 2-7. There can also be the splitting of a gluon into a quark-antiquark pair. Figure 2-8 shows an example of an even higher order process. Thus perturbative QCD theory predicts the cross section for two partons to scatter into some final state and inceasingly complex final states are described by increasingly higher order $\alpha_{\mathbf{S}}$ terms. 

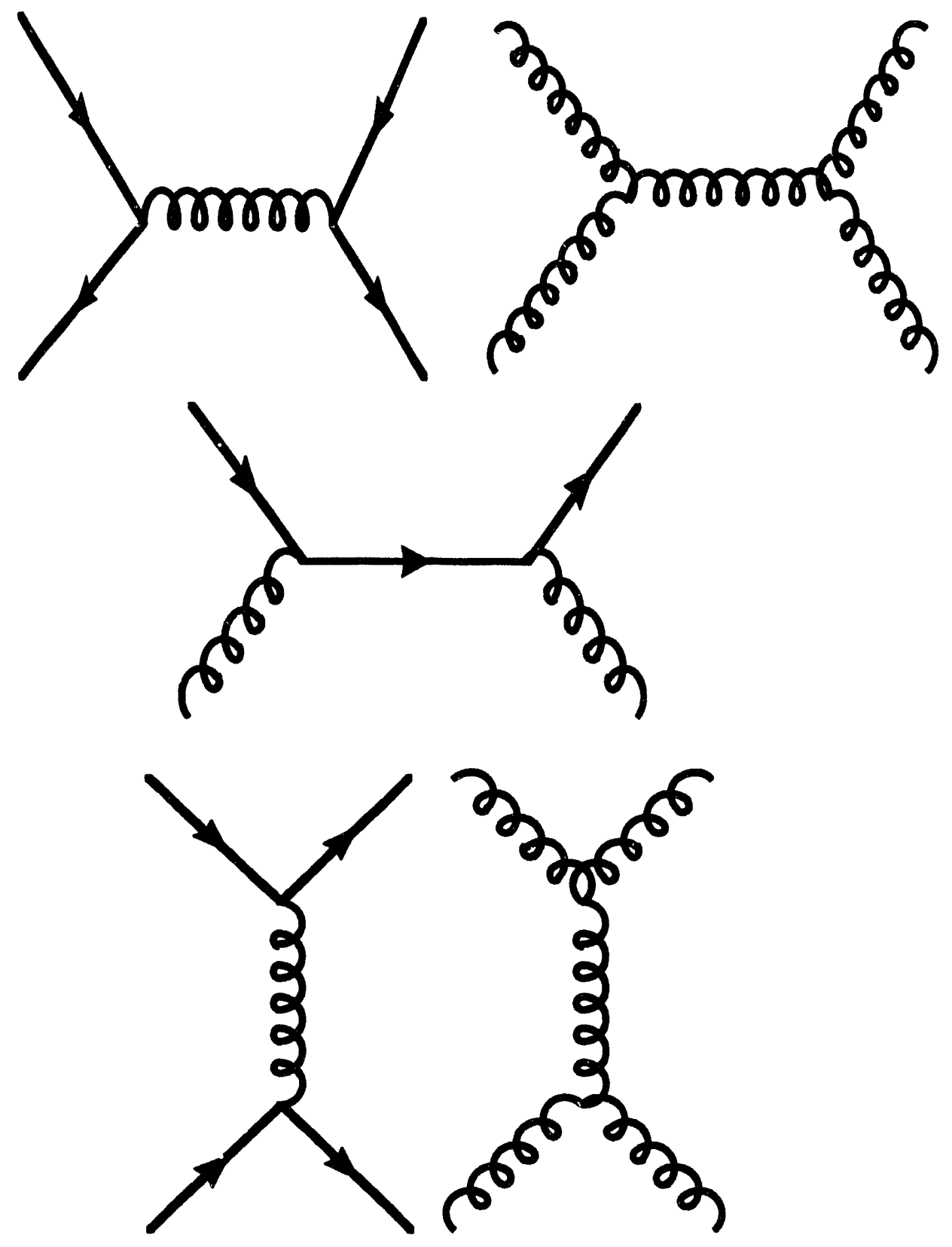

Figure 2-6: Example of a leading order $\alpha_{s}^{2}$ process. 


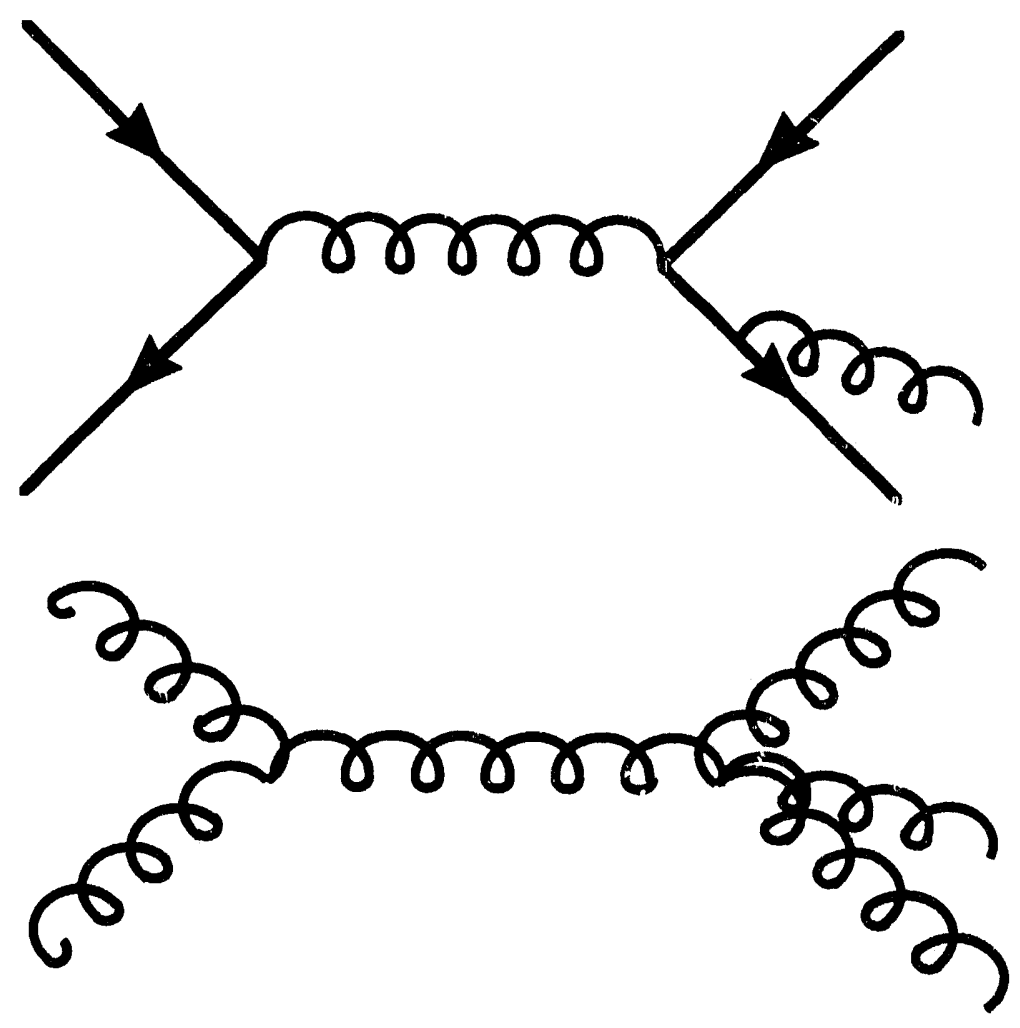

Figure 2-7: Example of a next-to-leading-order $\alpha_{s}{ }^{3}$ process.

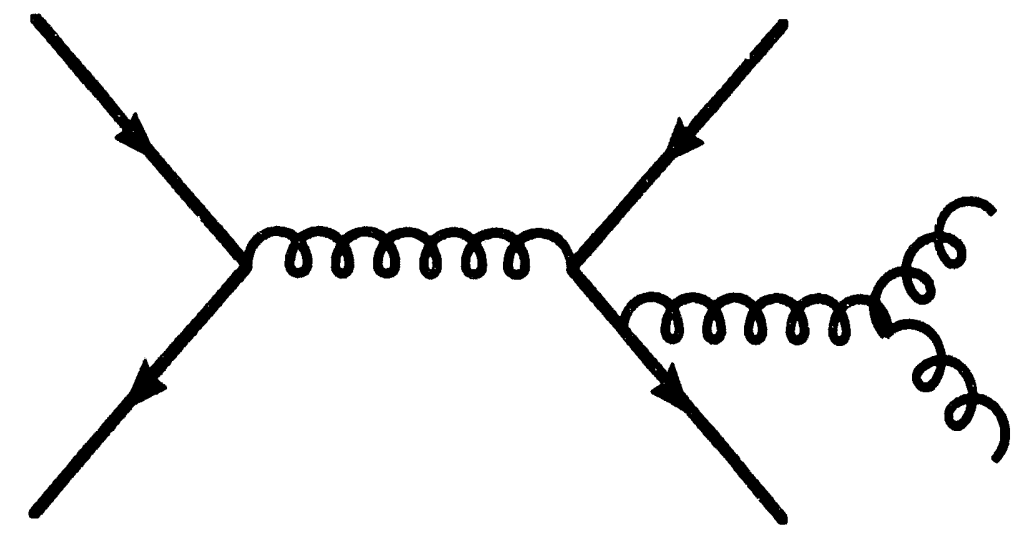

Figure 2-8: Example of a higher order $\alpha_{s}^{4}$ process.

\section{Parton-Parton Scattering Cross Sections}

Given the wide variety and complexity of partonic final states, the simplest class to consider is the inclusive final state which contains at least one parton. Note that this prediction must involve some quantity like the center of mass energy of the two initial parton system. First, we look at all the possible ways two colliding 
partons of specified type $(a, b)$ can form a partonic final state. This simplest configuration has two final state partons. To this contribution we add the contribution for 3,4 and higher final state parton multiplcities. We must sum over the types and colors of the final state partons and average over the initial state colors. Note that although the $2 \rightarrow 2$ contribution has contributions to all orders of $\alpha_{s}$, it is the only term that has $\alpha_{s}{ }^{2}$ terms. On the other hand, predictions calculated only to order $\alpha_{s}{ }^{2}$ predict only $2 \rightarrow 2$ interactions. The inclusive parton production for a specified 2 parton subprocess $(a, b)$ is the sum of all types of 2 parton processes (those with 2 parton final states, those with 3 parton final states etc...).:

$$
\begin{aligned}
& \sigma(a b \rightarrow \text { parton }+X)=2 C_{a b} \sum_{c, d} \sigma(a b \rightarrow c d)+3 C_{a b} \sum_{c, d, e} \sigma(a b \rightarrow c d e)+\ldots \\
& C_{q \bar{q}}=C_{q q}=C_{\overline{q q}}=\frac{1}{3^{2}}=\frac{1}{9} \\
& C_{88}=\frac{1}{8^{2}}=\frac{1}{64} \\
& C_{q g}=\frac{1}{3 \times 8}=\frac{1}{24}
\end{aligned}
$$

Here $\mathrm{X}$ denotes "anything else in the final state" and the C's are the color-averaging factors for the various parton pairs. c,d and e are summed over all types and colors of partons. We must also average over the initial spin states and sum over the final spin states.

\section{Parton distribution functions}

Since we can't experimentally isolate interactions that involved a particular parton pair $(a, b)$, we must sum over all possible pairs. To do this, we need some knowledge of the partonic structure of the proton as some partons occur with larger frequency than others. Additionally, the partonic cross sections shown in equation 2.2 have an intrinsic dependence on the invariant mass, $\mathbf{3}$, of the subprocess. However, the partons do not carry a fixed fraction of the proton's momentum. Thus $\$$ can vary from low values up to the $s$ of the proton-antiproton system which is (1.8 Tev) ${ }^{2}$. We need to know this distribution.

Parton distribution functions are probability distributions that describe the frequency that a parton occurs in a hadron with a certain fractional energy of the hadron. $x_{a}$ denotes the fraction of the hadron's total energy carried by parton a. The parton distribution function is denoted $f_{a} / A\left(x_{a}\right)$ and is the probability density 
parton a in hadron $\mathrm{A}$ with a momentum fraction $\mathrm{x}_{\mathrm{a}}$. For the case of protons it is more common and convenient to write:

$$
\begin{aligned}
& f_{q / P}\left(x_{q}\right)-q(x) \text { for quarks and } \\
& f_{g / P}\left(x_{g}\right)-g(x) \text { for gluons. }
\end{aligned}
$$

The valence quark composition of the proton and the requirement that the partons together carry all of the proton's momentum, give the following relations:

$$
\begin{aligned}
& \int u_{v}(x) d x=2 ; \int d_{v}(x) d x=1 \\
& \int x\left[g(x)+\sum_{q, \bar{q}}[q(x)+\bar{q}(x)]\right]=1
\end{aligned}
$$

Such functions must be measured. The quark distribution functions are measured from deep inelastic scattering of electrons and neutrinos on protons. Since these collisions involve electroweak interactions, the gluons cannot contribute. The gluon distribution functions can be determined, for example, from single photon experiments in which a gluon and a photon are produced in the final state.

Many sets of these parton distribution functions have been made using various fits to data from various experiments with various assumptions. Uncertainty in the p.d.f.'s tend to be the largest source of uncertainty in the cross section measurement. Figure 2-9 shows the up quark and gluon parton distribution function for two of these fits. Note that the up quark distribution peaks at $-10-15 \%$ of the proton's momentum. Both fits indicate that gluons dominate the soft portion of the distribution while the up quark dominates the region where a parton carries a large fraction of the proton's momentum. 


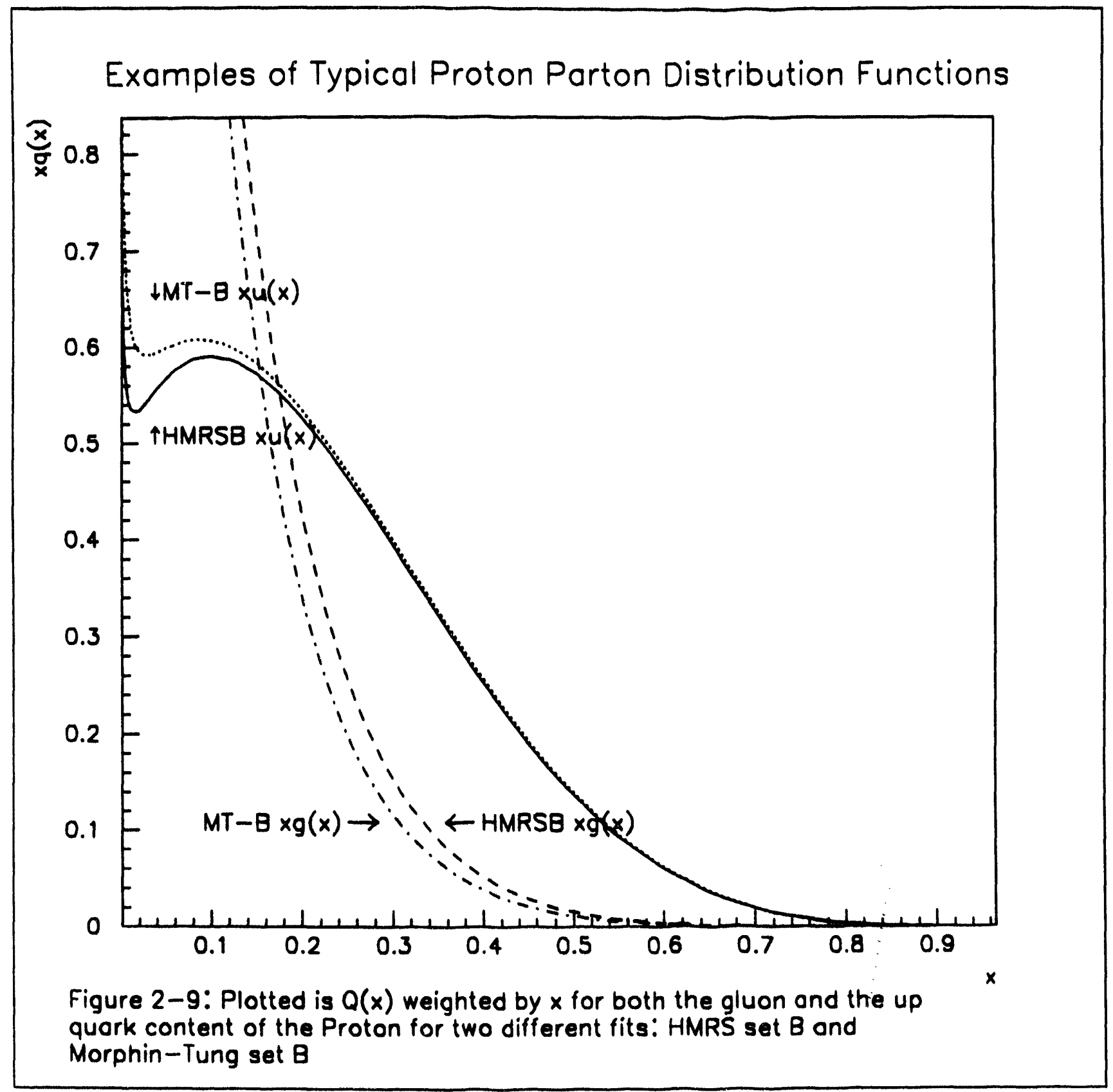




\section{Total Inclusive Jet Cross Section}

Thus we must use the p.d.f's in summing over the parton subprocesses to end up with a formula for the total inclusive cross section for parton scattering. We assume that a scattered parton will manifest itself as a jet:

$$
\begin{aligned}
\sigma(A B \rightarrow \text { jet }+X)= & \sum_{a, b} C_{a b} \int d x_{a} d x_{b} f_{a / p}\left(x_{a}\right) f_{b / \mathbb{P}}\left(x_{b}\right) \\
& x \hat{\sigma}(a b \rightarrow \text { parton }+X)
\end{aligned}
$$

$\mathrm{a}$ and $\mathrm{b}$ are summed over the $\mathbf{8}$ gluons and the three colored varieties of each flavor of quark. As already mentioned, the parton subprocesses cannot be calculated exactly. If we truncate our approximation at some order of $\alpha_{\boldsymbol{s}}$, we introduce an error that is manifested in an arbitrary QCD energy scale $\mu^{2}$. Theoretically, the cross section is independent of this parameter when calculated to all orders, but a dependence does appear when the calculation is done to some finite order and uncertainty in how to choose this scale then contributes to uncertainty in the calculation. Figure 2-10 shows the $\mu^{2}$ dependence of both the LO and NLO total inclusive cross section calculations. The NLO cross section is much less sensitive to the choice of $\mu^{2}$. All of our NLO and LO cross section calculations come from the calculations of Ellis, Kunszt and Soper combined with any one of a variety of parton distribution function sets. ${ }^{2}$ 


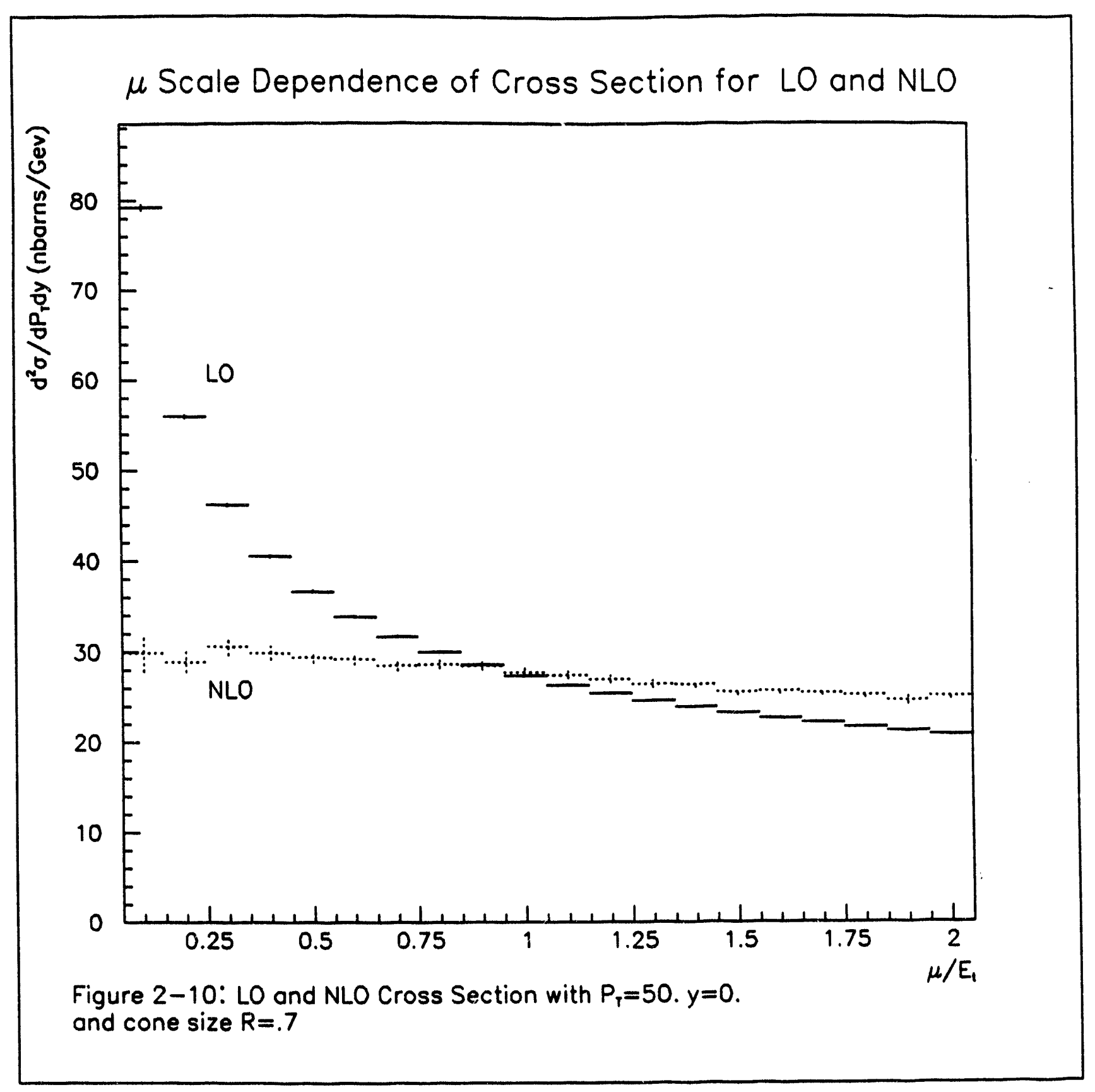




\section{Differential Jet Cross Section}

Although the total cross section is predicted by the theory, it is not the most useful quantity for comparing theory to experiment. One reason is that measurement of a scalar number is very sensitive to normalization errors. Additionally, it is experimentally difficult to measure jets over all phase space, especially for low jet energies and jets that are directed along the beamline. Therefore, predictions are usually made for the differential cross section with respect to some variable. The most popular choices are the transverse momentum $(\mathrm{PT})$ and the rapidity (y) of the jet. Rapidity will be defined later but it is strongly related to the angular direction of the jet with respect to the beamline. Both quanities are Lorentz invariant with respect to a momentum boost along the beamline which occurs whenever the $x$ of the colliding partons are not equal. The shape of these differential distributions is a more sensitive quantity to compare with experimental measurements. Figure 2-11 shows the resulting differential cross section for jet production using three different p.d.f. fits. The distributions show a dramatic fall as a function as $\mathrm{P}_{\mathrm{T}}$ as well as sensitivity to the choice of p.d.f. set. (which can changes portions of the spectrum by as much as $20 \%$ ) The impressive decrease in sensitivity to the choice of $\mu^{2}$ when using NLO as opposed to $\mathrm{LO}$ is shown in figure 2-12. Other experiments have already seen close agreement to the NLO prediction for this distribution ${ }^{3}$. DO will also be able to measure the rapidity distribution; an Ellis prediction for the rapidity distribution is shown in figure 2-13. 
Ellis NLO Inclusive Jet Cross Section vs $P_{T}$ for $y=0$

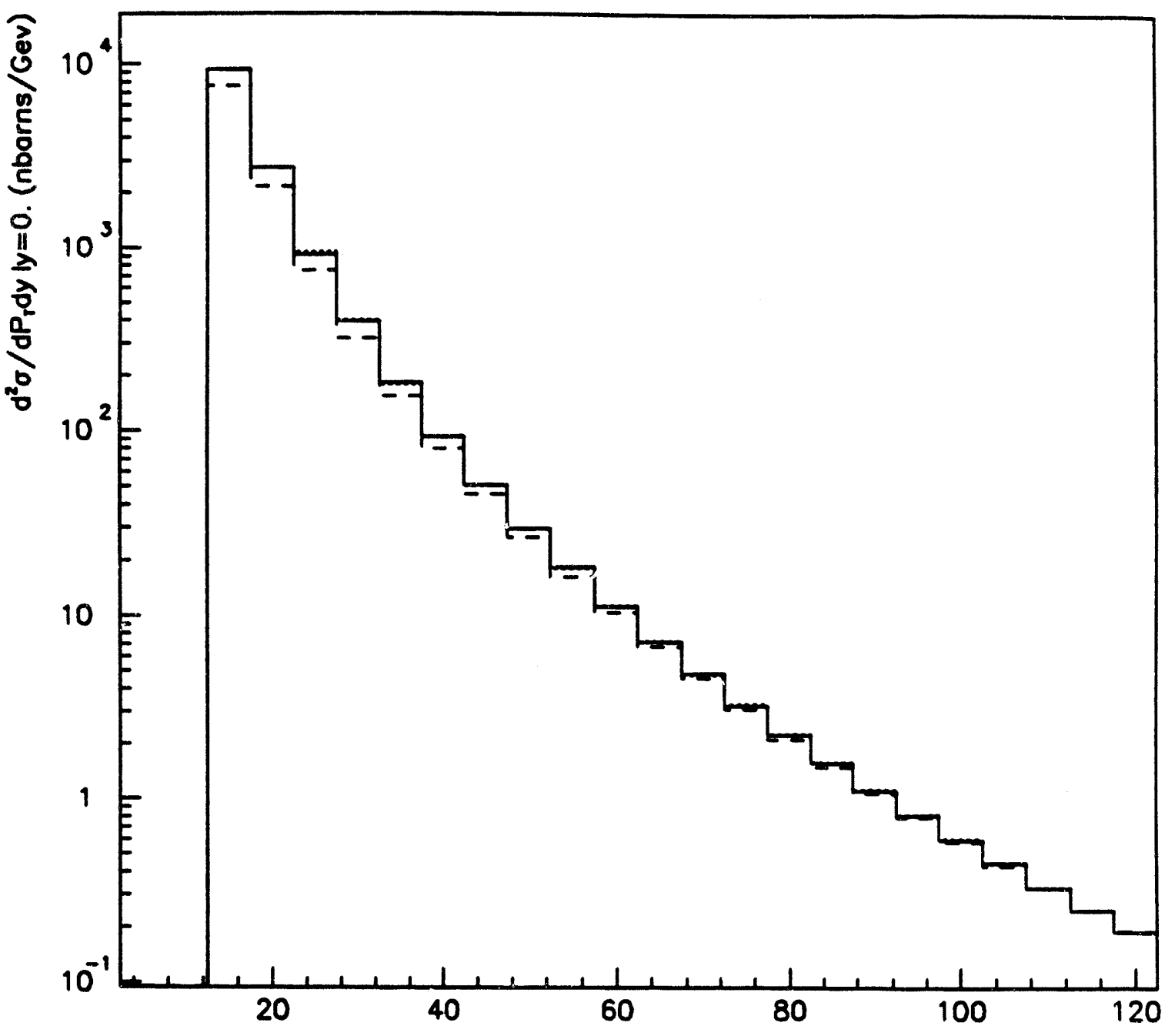

Figure 2-11: Shown are the next to leading order cross section spectrums from the Ellis program using various sets of proton distribution functions.

Solid,Dash and Dotted line correspond to HMRS-B, MRS-BO and MRS-B135 


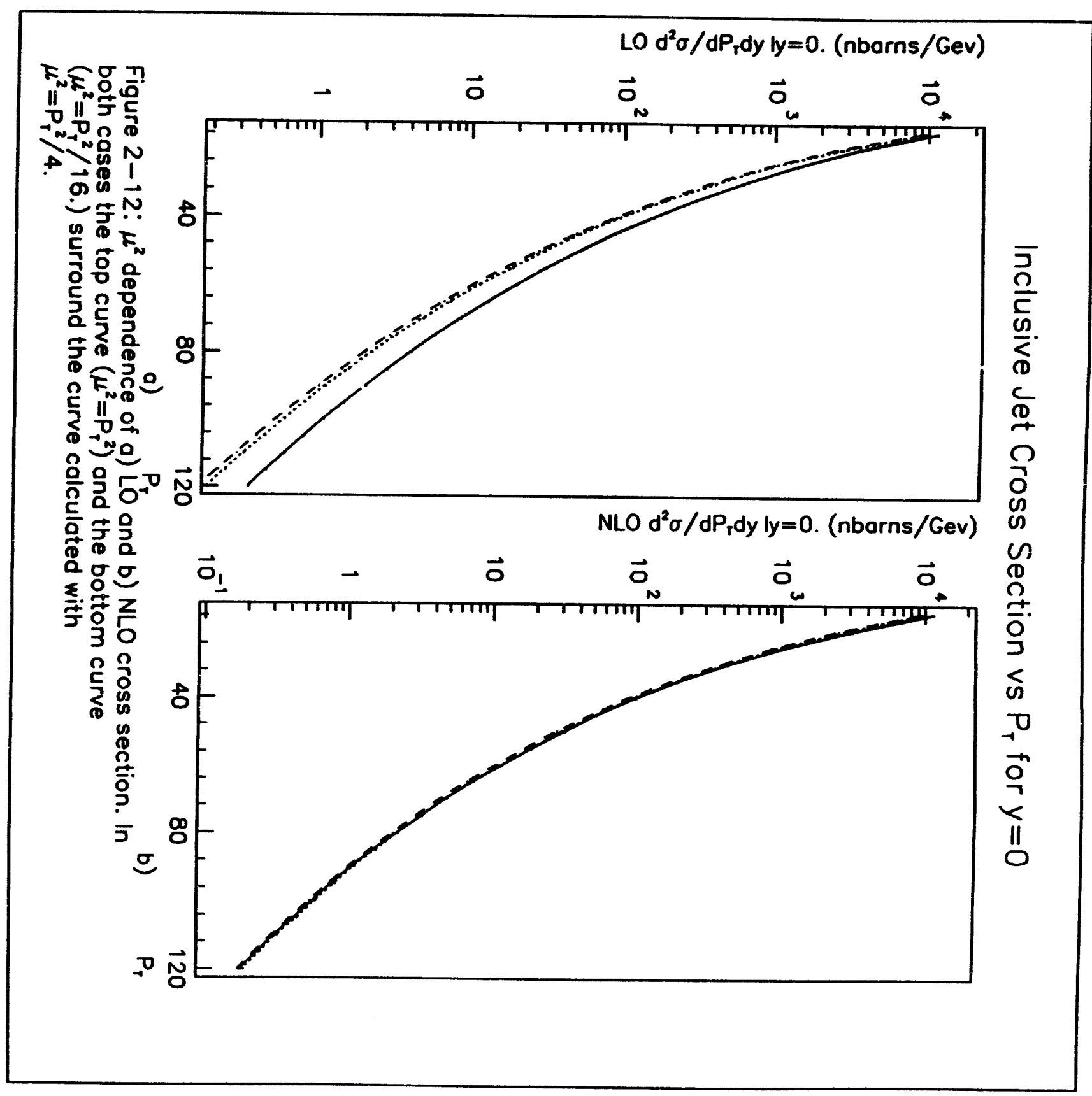




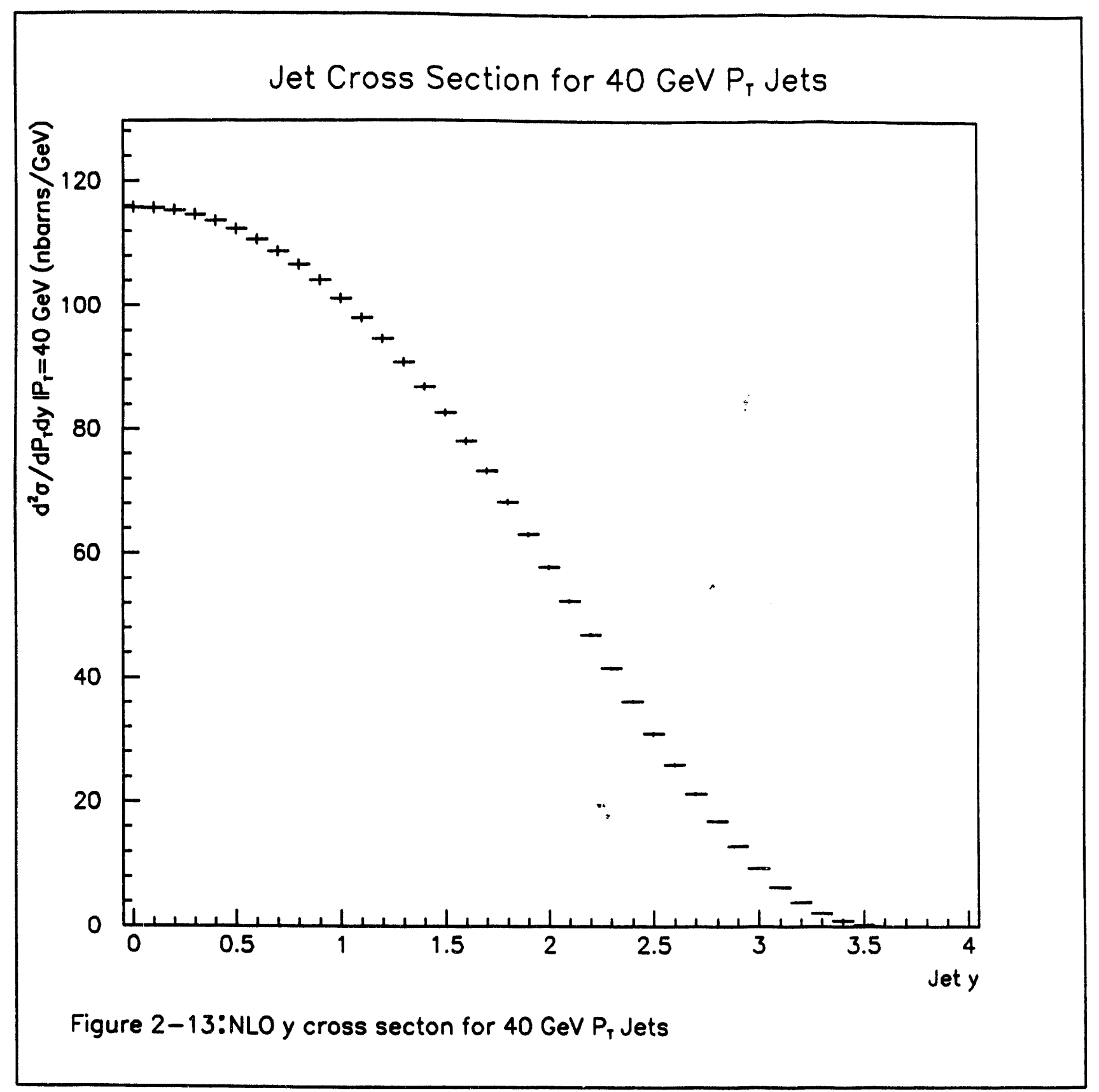




\section{The fall of the PT spectrum}

We can see qualitatively why the PT cross section falls so quickly by looking at the parton subprocess $\mathrm{gg} \rightarrow \mathrm{gg}$ which is the dominant subprocess for jets emitted transverse to the beam line. The cross section is related to the square of the matrix element; for this parton subprocess it is the following:

$$
M^{2}\left(g_{a} g_{b} \rightarrow g_{c} g_{d}\right)=\frac{9}{4}\left(4 \pi \alpha_{s}\right)^{2}\left(\frac{\hat{s}^{2}+\hat{t}^{2}}{\hat{u}^{2}}+\frac{\hat{s}^{2}+\hat{\mathrm{u}}^{2}}{\hat{\mathrm{t}}^{2}}+\frac{\hat{\mathrm{t}}^{2}+\hat{\mathrm{u}}^{2}}{\hat{\mathrm{s}}^{2}}+3\right)
$$

where $\hat{s}=\left(P_{a}+P_{b}\right)^{2} ; \hat{t}=-\left(P_{a}-P_{c}\right)^{2} ; \hat{u}=-\left(P_{a}-P_{d}\right)^{2}$

Assuming a massless gluon and evaluating the expressions in the center of mass frame:

$$
\begin{aligned}
& \hat{\mathrm{s}}^{2}=(2 \mathrm{E})^{4} ; \hat{\mathrm{t}}^{2}=\left(\mathrm{P}_{\mathrm{a}}^{2}+\mathrm{P}_{\mathrm{c}}^{2}-2 \mathrm{P}_{\mathrm{a}} \cdot \mathrm{P}_{\mathrm{c}}\right)^{2} ; \hat{\mathrm{u}}^{2}=\left(\mathrm{P}_{\mathrm{a}}^{2}+\mathrm{P}_{\mathrm{d}}^{2}-2 \mathrm{P}_{\mathrm{a}} \cdot \mathrm{P}_{\mathrm{d}}\right)^{2} \\
& \text { Using }\left(1-\cos \theta_{\mathrm{ac}}\right) \times\left(1+\cos \theta_{\mathrm{ac}}\right)=\left(1-\cos \theta_{\mathrm{ac}}^{2}\right)=\sin \theta_{\mathrm{ac}}^{2} \text { and }\left|\mathrm{P}_{\mathrm{a}}\right|=\left|\mathrm{P}_{\mathrm{c}}\right|=\mathrm{E}(2.6) \\
& \hat{\mathrm{t}}^{2}=\frac{1}{4} \hat{\mathrm{s}}^{2}\left(1-\cos \theta_{\mathrm{ac}}\right)^{2} ; \hat{\mathrm{u}}^{2}=\frac{1}{4} \hat{\mathrm{s}}^{2}\left(1+\cos \theta_{\mathrm{ac}}\right)^{2} \\
& \mathrm{M}^{2}\left(\mathrm{~g}_{\mathrm{a}} \mathrm{g}_{\mathrm{b}} \rightarrow \mathrm{g}_{\mathrm{c}} \mathrm{g}_{\mathrm{d}}\right)=\frac{9}{4}\left(4 \pi \alpha_{\mathrm{s}}\right)^{2}\left(\frac{2 \hat{\mathrm{s}}^{2} \hat{\mathrm{t}}^{2}+\hat{\mathrm{t}}^{4}+\hat{\mathrm{u}}^{4}}{\frac{1}{16} \hat{\mathrm{s}}^{4} \sin ^{4} \theta_{\mathrm{ac}}}+\frac{\hat{\mathrm{t}}^{2}+\hat{\mathrm{u}}^{2}}{\hat{\mathrm{s}}^{2}}+3\right) \\
& =\frac{9}{4}\left(4 \pi \alpha_{s}\right)^{2}\left(\frac{\frac{1}{2}\left(1+\cos ^{2} \theta_{\mathrm{ac}}\right)+\frac{1}{16}\left(1-\cos \theta_{\mathrm{ac}}\right)^{4}+\frac{1}{16}\left(1+\cos \theta_{\mathrm{ac}}\right)^{4}}{16} \sin _{\mathrm{ac}}\right. \\
& \left.\quad+\frac{1}{4}\left(1-\cos \theta_{\mathrm{ac}}\right)^{2}+\frac{1}{4}\left(1+\cos \theta_{\mathrm{ac}}\right)^{2}+3\right) \\
& =\frac{9}{4}\left(4 \pi \alpha_{\mathrm{s}}\right)^{2}\left(\frac{\left.10+4 \cos ^{2} \theta_{\mathrm{ac}}+2 \cos \theta_{\mathrm{ac}}+\frac{2}{4}\left(1+\cos _{\mathrm{ac}}^{2}\right)+3\right)}{\sin ^{4} \theta_{\mathrm{ac}}}\right.
\end{aligned}
$$

We insert this $\mathrm{M}^{2}$ into a familiar formula for the differential cross section and recognize that the first term dominates for smaller angles:

$$
\frac{\mathrm{d} \hat{\sigma}}{\mathrm{d} \hat{\mathrm{t}}}=\frac{\mathrm{d} \hat{\sigma}}{\mathrm{dP}_{\mathrm{t}}^{2}} \frac{1}{\cos \theta_{\mathrm{ac}}}=\frac{1}{16 \hat{s}^{2}}|\mathrm{M}|^{2} \equiv \frac{1}{64} \frac{f(\theta)}{\mathrm{P}_{\mathrm{t}}^{4}}
$$


Thus the parton subprocess by itself predicts a cross section that falls quickly as a function of PT. Normally, the PT distribution is calculated with the angle between the outgoing quark and the incident direction fixed. In that case the distribution is dependent on P. But the angle is fixed in the laboratory frame and not the center of mass frame of the partons. We will try to estimate the $\mathrm{PT}$ dependence when we require the outgoing parton to be completely transverse in the laboratory frame. By constraining the angle, the $\mathrm{PT}$ dependence is essentially a dependence on $\mathrm{P}$ and $\mathrm{P}^{2}$ is proportional to 3 .

$$
\frac{d \hat{\sigma}(p \bar{p} \rightarrow j e t+x)}{d P_{t}^{2}} \approx \int d x_{a} d x_{b} g\left(x_{a}\right) g\left(x_{b}\right) \frac{1}{16 \hat{s}^{2}}\left|M^{2}\right|
$$

and

$$
\hat{s}=\left(\left(x_{a} \sqrt{s}+x_{b} \sqrt{s}\right)^{2}-\left(x_{a} \sqrt{s}-x_{b} \sqrt{s}\right)^{2}\right)=4 x_{a} x_{b} s
$$

Experimental fits show that:

$$
g(x) \propto \frac{1}{x}(1-x)^{6}
$$

Thus the integrand is proportional to:

$$
\left(1-x_{a}\right)^{6}\left(1-x_{b}\right)^{6} \frac{1}{\hat{s}^{3}}
$$

Since the angle is relatively fixed, the only way to increase $\mathrm{PT}$ is to increase the $\mathrm{x}$ values and thus this term falls faster than $1 / \mathrm{P}^{6}$. Thus after the integral is done, we might expect the differential distribution to fall like PT to some power. Fits to the calculated spectrum show that:

$$
\frac{\mathrm{d} \sigma}{\mathrm{dP}_{\mathrm{t}}} \propto \mathrm{P}_{\mathrm{t}}^{-6}
$$

Although the fall of the ET spectrum provides a more sensitive test of the $Q C D$ predictions than a softer spectrum, it does make the measurement of the spectrum more difficult. There is always some systematic error in the measurement of the jet $\mathrm{Pt}$ at the experimental level. For a given $\triangle \mathrm{PT}$, the consequential bias in the cross section might be expected to be: 


$$
\frac{d \sigma}{\sigma}=-N \frac{d P_{t}}{P_{t}} \text { if we assume } \sigma \propto P_{t}^{N}
$$

Thus our fractional error in measuring the cross section is magnified $\mathrm{N}$ times by our fractional error in measuring the jet $\mathrm{PT}$

Even if the jet measurement was made without bias, such that the PT was measured correctly on the average, there is always an effect due to the finite PT resolution combined with the steeply falling cross section. Finite resolution means that jets that belong in one Pt bin will sometimes be mismeasured enough to be deposited in a higher or lower bin. To some extent, neighboring bins compensate the losses of each other, but if the spectrum falls fast enough, each bin receives many more entries from the more populated bins than it gives to the less populated bins. Hence the distribution becomes 'smeared' and thus the measured spectrum must be unsmeared at some point.

\section{Fragmentation of quarks into jets}

Our attempt to measure the inclusive jet cross section depends on our ability to infer the energy and direction of the original parton with the jet of fragmented particles which we see in the detector. This process cannot be calculated perturbatively at present; instead a fragmentation scheme is used by the generator to simulate jets in the detector. Our estimate of how well we can determine the attributes of the parton jet from the particle jet may depend on which scheme is used.

One type of fragmentation scheme is an 'independent fragmentation scheme' in which the scattered parton is fragmented in a manner independent of the other present partons. This method was first presented by Feynman and Field 4 . Other methods do consider the other partons in the proton. For example, string fragmentation methods describe the partons as being connected by strings which may break and form quarkantiquark pairs 5 . Figure 2-14 depicts a very simple model of how the breakup of a proton and subsequent fragmentation of the quarks might look in its center of mass frame. In b) the valence quarks begin to separate and as they do, the color field lines are become collimated between the quarks and the rest of the system. In c) the quarks move further apart and the potential energy of the field grows almost linearly with the separation until d) it becomes energetically favorable for quark-antiquark pairs to be created. These pairs can form colorless mesons in e) and move off as a jets of particles in $\mathrm{f}$. 

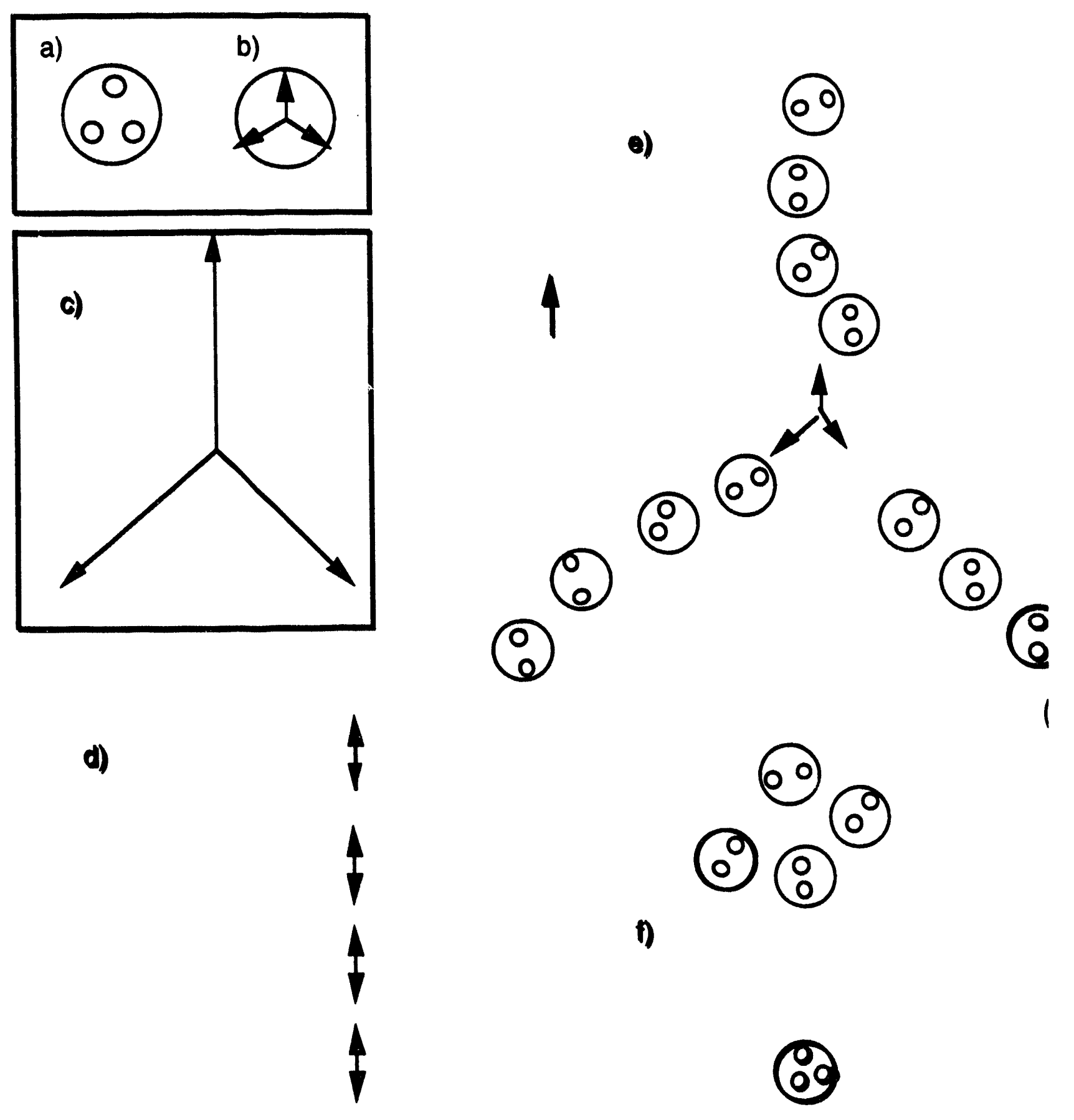

d)

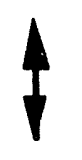

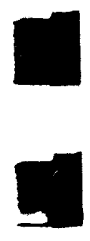
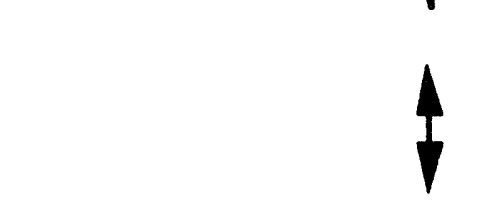

1

)
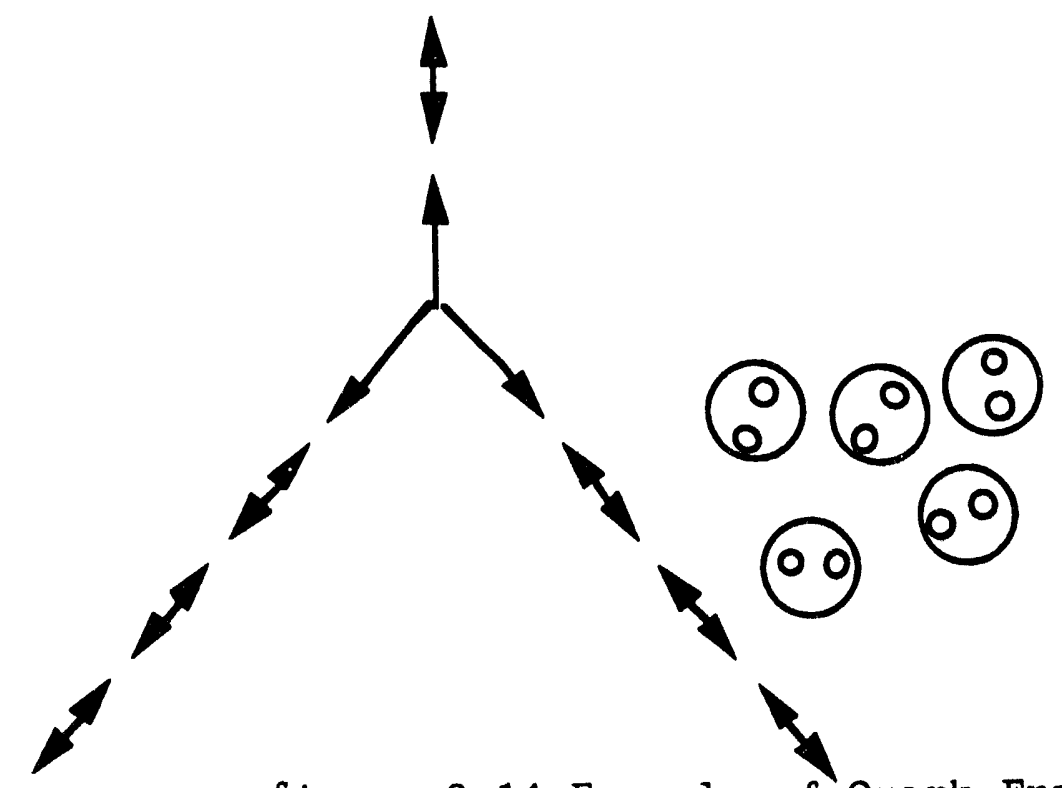

figure 2-14 Example of Duark Fragmentation

0

(0) 
1V. Barger and R. Phillips, Collider Physics, Addison-Wesley, Redwood City, CA (1987)

${ }^{2}$ Calculations obtained via a software routine which would make the calculations using one of many sets of parton distribution functions supplied by Morhin-Tung (J.

Morphin, W-K. Tung), HMRS (P. N. Hariman, A. D. Martin, R. G. Roberts, W. J. Stirling), and MRS ( A. D. Martin, R. G. Roberts, W. J. Stirling)

${ }^{3}$ CDF Collaboration, Inclusive Jet Cross-Section in Anti-P $P$ Collsions at sqrt $(S)=1.8$ TeV, FERMILAB-Pub-91-231 (1991) (submitted to Phys. Rev. Lett.)

${ }^{4}$ R. D. Field and R. P. Feynman, A Parametrization of Quark Jets, Nucl. Phys. B239, 349 (1984)

${ }^{5}$ B. Andersson et. al., Parton Fragmentation and String Dynamics, Phys. Rep. 97, 147 (1983) 


\section{Chapter 3 : Description of Computer Simulations Used}

All work presented here has been the results of extensive computer simulations and analysis of the results of such simulations. We will describe the various simulations used and, where possible, point out some of their limitations. The simulations fell into two categories, the first of which is Event Generation in which the collision of a proton with an antiproton at the expected center of mass energy of 1.8 $\mathrm{TeV}$ is simulated. The second category is known as Detector Simulation. Here the output from the Event Generation was combined with detector geometry and selected physics processes to simulate the response of the detector. The results were written in a format similar to that produced by the D0 Data Acquisition System. Finally, reconstruction and analysis routines were run on the output of the first two. For the most part, this software is identical to what will be used on real data.

\section{Event Generation}

Although various event generators have been used for comparison's sake, the ISAJET event generator was used primarily. ISAJET simulates proton-antiproton interactions at $\sqrt{\mathrm{s}}=1.8 \mathrm{TeV}$. We will briefly summarize the features of the program. First, a parton hard scattering is generated using a parameterized perturbative QCD cross section. All quarks are assumed to be massless except the top and bottom quarks. This is always a $2 \rightarrow 2$ process. The user can set kinematic limits on the energy and angular direction of the partons in the final state as well as the types of partons that are in the initial state. Such limits only apply to the two partons that result from the initial $2 \rightarrow 2$ process. To approximate higher order QCD, radiative corrections in which either the initial or final state partons may emit gluons are made. Each quark or gluon has a chance to emit a gluon. This probability is proportional to a splitting function multiplied by $\alpha_{s}$. The same probability exists for a gluon to split into a 
quark-antiquark pair. The procedure is reapplied to the daughter quarks and gluons. In this way, ISAJET approximates higher order processes by producing events with 3 and more final state partons. The process is terminated once the parton falls below 6 $\mathrm{GeV}$ in energy. At this point, the partons are fragmented into particles using a FieldFeynman fragmentation scheme ${ }^{1}$. Particles with a lifetime of less than 10-12 are decayed inmediately. This finishes the simulation of the hard scattering. The beam jets, which represent the remains of the fragmented proton and antiproton, are added by simulating an interaction in which no hard scattering took place. This event is superimposed on the hard scattering event. This process conserves transverse momentum, but not energy. Thus the event energy is rescaled at the end. The vertex of the event is selected with a Gaussian generator which is centered around the nominal interaction point with a width of $30 \mathrm{~cm}$. This is expected to simulate the real distribution of collision points from the Tevatron. At the end of the event generation phase we are left with two lists of 4 vectors; one for final state partons and one for the final state particles. Note that the event simulation does not include events in which there was more than one proton-antiproton collision and it does not include the possibility of simultaneous cosmic rays. An associated piece of software that often ran after event generation was a jet finder named PJETS ${ }^{2}$. PJETS tried to identify jets from the generator's list of final state partons or particles. Such jets are referred to as 'generated' jets and will be discussed further in a later chapter.

\section{Detector Simulation}

Detector Simulation is the most complicated and time consuming portion of the simulation process. It combines detailed knowledge of the geometry and makeup of the D0 detector with simulated physics processes to determine the response to high energy particles streaming outward from the vertex point. The detector is represented by nested volumes within volumes. Each volume is meant to approximate the size and material makeup of a real detector piece. If this were done precisely, the resulting geometry would require large amounts of computer time to process. Therefore approximations had to be made. Since jet reconstruction was dependent almost entirely on the calorimeter simulations, we will limit our discussion to that portion of the detector simulation. The three large calorimeters which consist of alternating layers of uranium, readout boards and gaps filled with liquid argon were too intricate to be included. (See chapter 4) Therefore, they were replaced by a 'mixture' material whose density approximated that of the real structure. Still maintained after this 'simplification' is the gap between the central and endcap calorimeters, the walls of the 
cryostats which contain the calorimeters and the phi cracks which are between the modules of the central calorimeter. The GEANT physics program was used to simulate particles in the detector. Physics processes such as Compton scattering, pair production, photo electric effect and photo fission as well as multiple scattering, ionization, delta ray production, bremsstrahlung, nuclear and hadronic interactions were simulated. Detector response is calculated in terms of energy loss in 'active' portions of the calorimeters. Output data banks which are similar to those produced by the real data acquisition system were made. Since each particle that moves through the calorimeter loses only a small fraction of its energy in any physics process, the simulation is slowed considerably if the processes are allowed to continue indefinitely for any particle. Normally an energy cutoff is set which requires that a particle deposit all of its energy once it falls below this cut off. This is especially important in the predicted transverse shape of electromagnetic showers in the detector. The real calorimeter channels all have 'noise' distributions which come from the electronics and real argon ionization from decays in the uranium plates. Noise means that an empty calorimeter cell outputs a finite signal that often follows some Gaussian-like distribution with irregular tails. The detector simulation had no noise simulation in it. A simulation of the detector's trigger system was available but it was not used to sele.jt events for these studies and will only be discussed in the chapter on jet triggering.

\section{Event Reconstruction}

Finally, offline reconstruction and analysis routines were run. These routines are the same as those that will be used on real data and were evolved into their present form through this simulation process. Although a vertex reconstruction routine was available, most of the studies done used the vertex supplied by the event generator. Therefore, additional errors in reconstructed jet quantities due to vertex mismeasurement are not included in those studies. An offline jet reconstruction routine named CAJETS $^{3}$ was user to reconstruct jets. Results of this routine will be referred to as 'Reconstructed' jets. 
IIBID. Field and Feynman.

2B. Klima and C. Stewart, PJET-Parton Jets in the Monte Carlo. D0 Note 905, D0 Experiment at Fermilab, Batavia, IL, December 1989.

3N. J. Hadley, Cone Algorithm for Jet Finding. D0 Note 904, D0 Experiment at Fermilab, Batavia, IL, November 1989. 


\section{Chapter 4: DO Detector and Jets}

Jets are characterized by a spray of energetic, high ET particles in a localized area of space. This definition is vague, but the truth is that jets are not well defined objects. We will see that even at the parton level, what constitutes a jet is ambiguous. For now, the above definition is enough to make apparent the challenges of reconstructing jets. We have seen that the theory can make predictions about 1) The transverse energy of the jet and 2) The rapidity of the jet. Therefore, our detector should be able to 1) reconstruct the energy of the jet 2 ) reconstruct the vertex of the event and 3) identify the direction in space of the jet. The last two together allow the polar angle, $\theta$, of the jet axis with respect to the nominal beamline to be reconstructed We will describe the various components of the $\mathrm{D} 0$ detector, paying special attention to those components that aid in accomplishing any one of these three objectives. We will also mention to what degree they were simulated for our studies.

\section{Level O Scintillators 1}

There are 2 sets of scintillators, one on each side of the nominal interaction region, offset about $130 \mathrm{~cm}$, which comprise the Level 0 . Each set covers an angular cone of about 10 degrees around the beamline. (covering $\eta$ from 2.2 to 4.1 ) Whenever the proton fragments, particles are thrown transverse to the beamline. These scintillators are intended to detect such particles and hence flag those interactions in which breakup of the proton or antiproton occurs. The Level 0 was not simulated for this analysis. The Level 0 scintillators are labeled in figure 4-1. The primary role of the Level 0 is that of a trigger element and it will be discussed further in the chapter on jet triggering. 


\section{Central Detector ${ }^{2}$}

The Central Detector (CD), fills a barrel shaped region that surrounds the beam pipe and extends radially out to $84 \mathrm{~cm}$ and out to $\mathrm{z}=130 \mathrm{~cm}$ on both sides of the nominal interaction point. Primarily, the $\mathrm{CD}$ consists of three concentric regions flanked by two identical sections of a fourth type as shown in figure 4-1. Most of these regions contain drift chamber cells. Drift chambers are detectors that measure the trajectory of charged particles. A charged particle ionizes the drift chamber gas and these ions 'drift' under the influence of an applied electric field. Position and drift time measurements can be used to reconstruct 'hit' positions along this path which in turn are fitted to produce the trajectory of the path itself. Assuming that the number of such ionizations are proportional to the energy deposited by the particle allows the determination of its $\mathrm{dE} / \mathrm{dx}$ (energy loss per unit length). The $\mathrm{dE} / \mathrm{dx}$ of an electron differs from that of a photon which converts into a electron-positron pair allowing discrimination between the two. Unlike some detectors, the $\mathrm{D} 0$ central detector lacks a central magnetic field, this means that the momentum and sign of the charged particles cannot be determined.

Vertex Chamber: The vertex chamber is closest to the beam-pipe and reconstructs tracks around the interaction point. Its job is to measure the interaction point. This is crucial in determining the PT of the jet.

Transitional Radiation Detector (TRD): The purpose of the TRD is to discriminate electrons from charged pions by measuring the transitional radiation produced when a charged particle crosses a dielectric interface.

Central Drift Chamber: (CDC): The CDC reconstructs trajectories of charged particles from the interaction region.

In addition, there are two drift chambers (one forward and one backward of the other central detector elements) that fill the entire radial region from the outside of the beam pipe to the outer radius of the other central detector elements. These are the Forward Drift Chambers (FDC).

Although simulations of these detector elements exist, jet reconstruction for the most part is only concerned with the location of the vertex. In most of the studies described in the following chapters, the vertex was taken from the event generator instead of from the reconstruction. Thus the added uncertainty in jet PT derived from mismeasurement of the vertex is not included in those analysis. However, the vertex reconstruction was used in the chapter on Jet Reconstruction and in the high statistics sample with which we reconstructed the jet cross section in the Results chapter. 


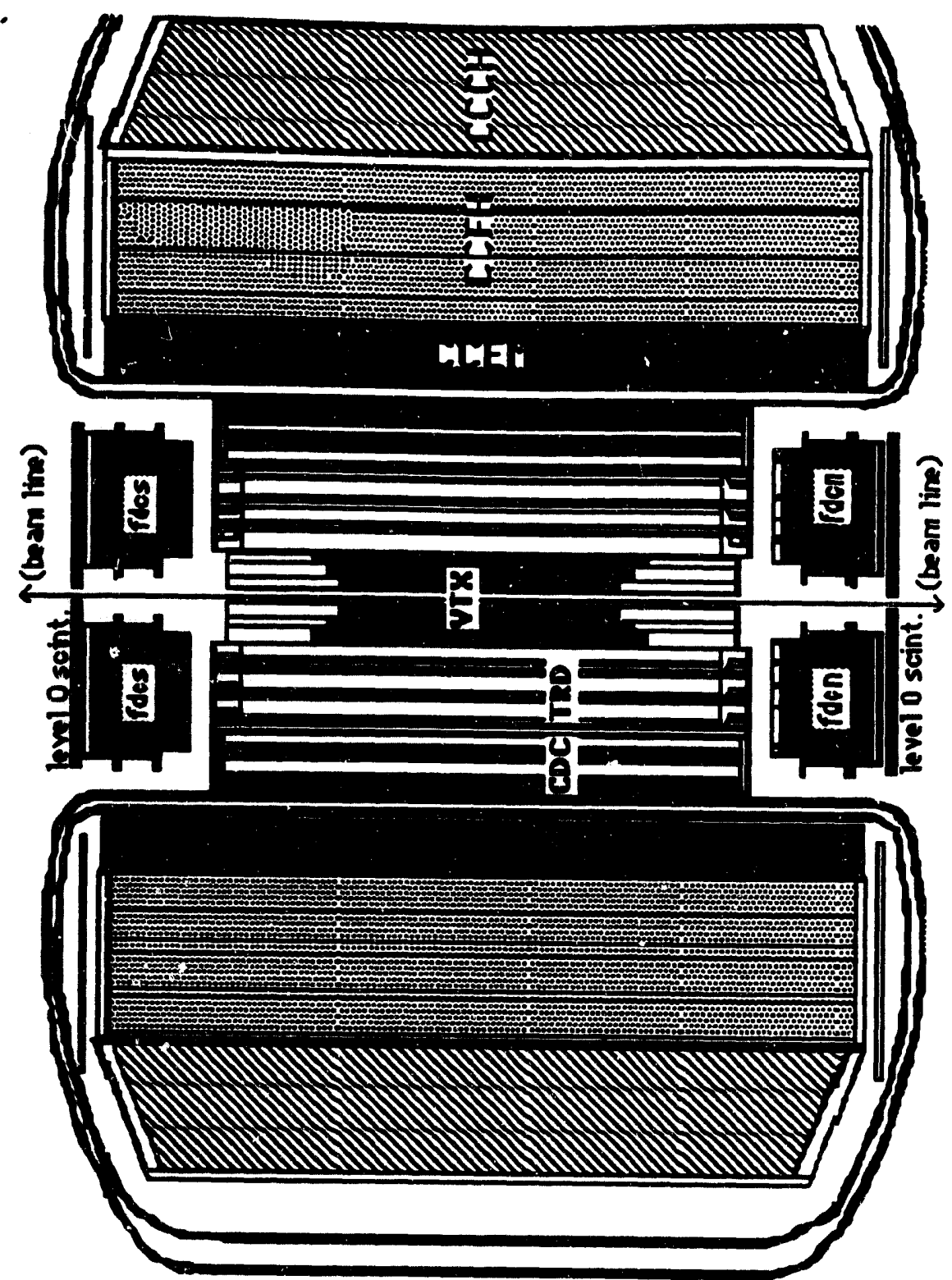

Figure 4-1: Central Detector, Central Calorimeter and Level 0 Scintillators are shown in this slice view of the central portion of the detector. The arrow marks the beam line.

\section{DO Calorimeters ${ }^{3}$}

The Central Detector is unable to provide either momentum or energy measurements of w.e particles that pass through it. In fact, it does not see nevitral particles at all. It is the job of the DO calorimeters to contain and measure the energy of all the particles produced in the collision except for muons. They must also provide spatial resolution to allow determination of transverse energy and the separation of jets from each other. Thus the calorimeter is the most important 
detector element for jet reconstruction. D0 has three calorimeters: a central calorimeter and two endcap calorimeters, one on each side of the central calorimeter.

To measure as much of the event as possible and to identify broad jets, the calorimeters must be both hermetic and containing. Figure 4-2 shows a side view of the calorimeters. The calorimeters are symmetric in $\phi$ and provide coverage down to a pseudorapidity of 4.1. This corresponds to 2 degrees from the beam line. Hence the calorimeters cover nearly $4 \pi$ in solid angle. The calorimeters should provide containment for hadrons as well as for electrons and photons. The central calorimeter electromagnetic section (labeled CCEM in figure 4-2) contain 20 radiation lengths of material. In the end calorimeters, there is an electromagnetic section (ECEM) located just inside the End Cap Inner Hadronic (ECIH) which provides similar electromagnetic containment. For hadrons, each calorimeter contains several sections of hadronic calorimetery. The central calorimeter has a uranium fine hadronic (CCFH) section followed by a coarse hadronic ( $\mathrm{CCCH}$ ) section which is made of copper plates. The endcap has two sections (ECIH and ECMH) outside of the ECEM, which have both a fine uranium section and a coarse stainless steel section) and an outer ring of stainless steel modules referred to as the outer hadronic (ECOH) section. The central detector provides 7 interaction lengths of material while the end calorimeters provide up to 9 interaction lengths. 


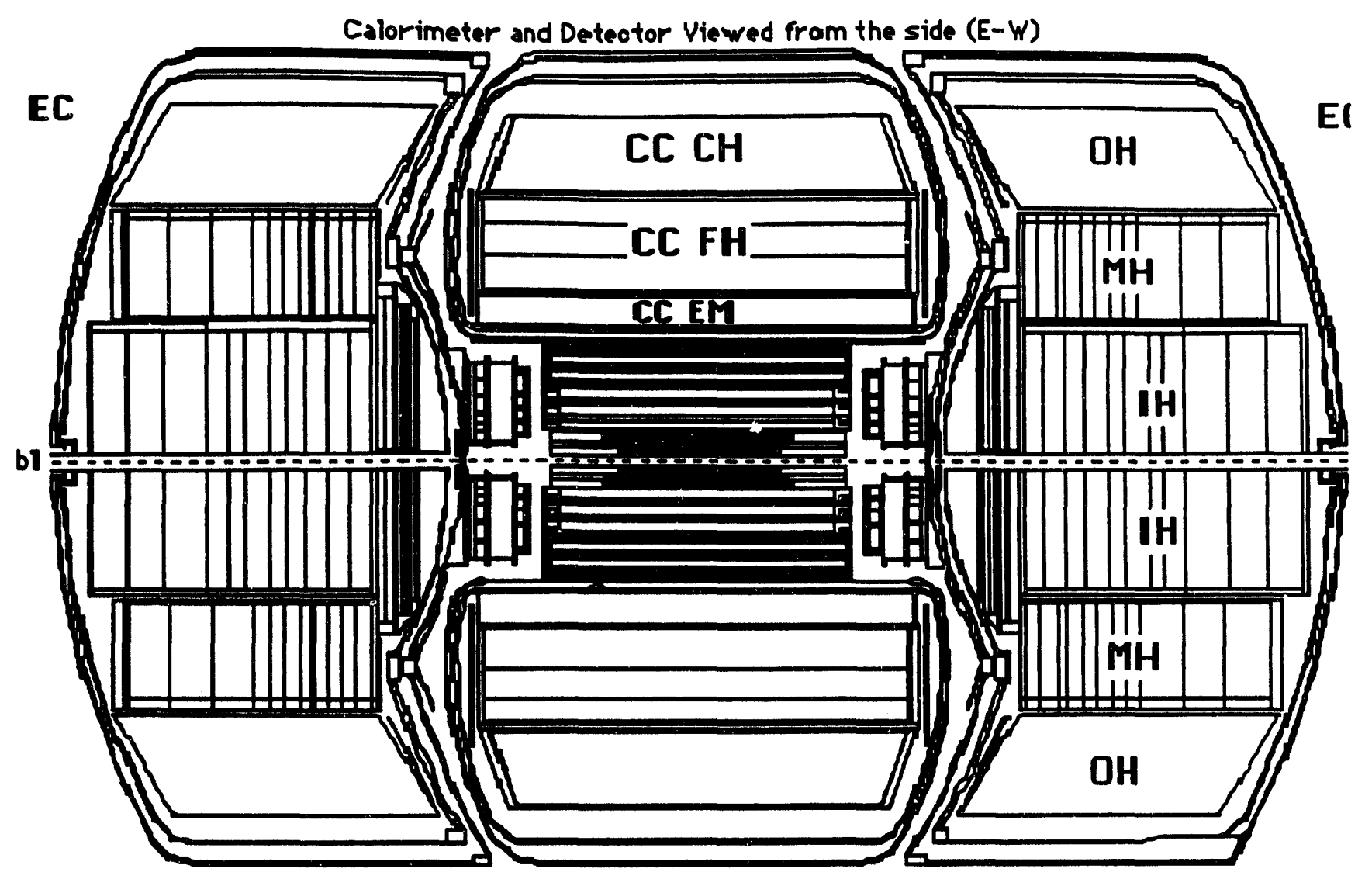

Figure 4-2: The three calorimeters of the D0 detector.

Spatial resolution is also important to allow one to distinguish among nearby jets and to measure the transverse energy of the jet. The calorimeters are divided into various read out channels that segment the calorimeter azimuthally in $\phi$, in pseudorapdity and longitudinally outward from the nominal interaction point. Thus the readout channels are divided into projective 'towers' that occupy .1x.1 in $\phi$ pseudorapidity space. Pseudorapidity will be defined later but is related to the polar angle that the tower makes with respect to the beam line. These towers become physically smaller in the forward and backward regions of the detector as shown in figure 9-2. The calorimeter is divided into $64 \phi$ sections, 74 pseudorapidity bins and about 14 longitudinal layers in each tower for an approximate total of sixty-six thousand readout channels. The central calorimeter is separated into many phi modules each of which span the length of the central calorimeter. Although the CCEM and CCFH modules are staggered so that their phi edges do not align, there are cracks in between modules. This is also true of the ECMH and ECOH rings. The 
ECEM and ECIH are one piece modules and hence do not have cracks in either $\phi$ or $\eta$. The only pseudorapidity cracks are between the calorimeters. Special detectors (see the next section) are installed in these regions to measure energy deposited in this region. There is transverse segmentation for both the electromagnetic as well as the hadronic sections. The electromagnetic sections have four sections and the third one (which is expected to contain from $50 \%$ to $80 \%$ of the energy of an electromagnetic shower) is twice as finely segmented (.05 $\times .05$ in $\eta-\phi$ space) as the rest of the detector. The hadronic section has three layers of uranium calorimetry and a final layer of copper calorimetry in the central calorimeter and four uranium layers and a couple stainless steel sections in the endcap calorimeters.

Each readout channel is divided up into several readout cells whose signal is summed. For the most part, each readout cell looks like every other readout cell in the three calorimeters. Each cell consists of a liquid argon filled gap sandwiched by an absorber plate (usually uranium) and a signal board. Each calorimeter is contained by a cryostat which holds and preserves the liquid argon. The dense absorber plates cause the breakup and showering of particles that pass through. Although most of the particle's energy is deposited in the absorber, some of it is lost in the liquid argon gap in the form of ionized argon atoms. The fraction of energy deposited here is expected to be a fixed fraction of the overall energy deposited. An electric potential between the absorber plate and the resistive surface of the signal board moves the liberated electrons across the gap and induces an electric current which can be determined by measuring the voltage difference across a capacitor This signal is amplified, baseline subtracted and digitized. Uranium-liquid argon calorimeters have been found to have a response that is more linear with energy and less sensitive to particle type than other designs. ${ }^{4}$ 


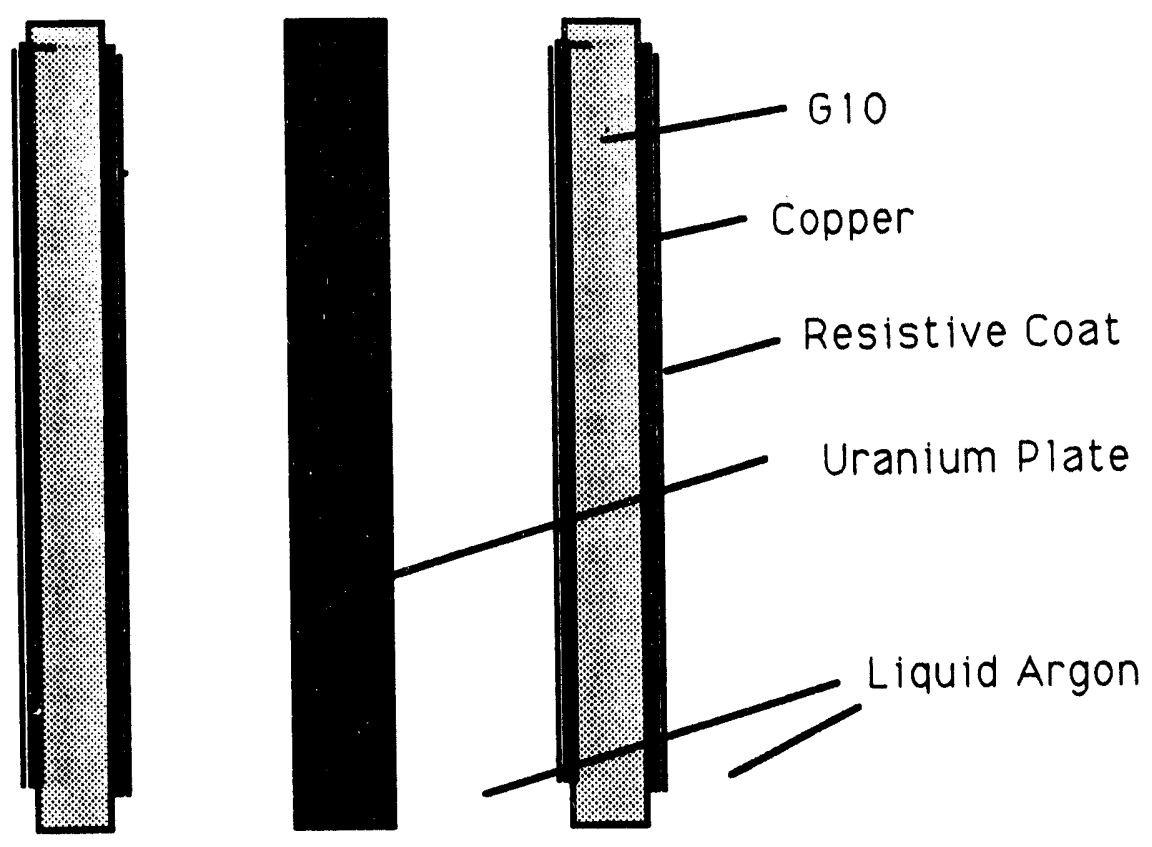

Figure 4-3: Sample Calorimeter Readout Cell

\section{Inter-Cryostat and Massless Gap Detectors}

As mentioned above, energy deposited within the calorimeter is determined by measuring the charge within an argon gap and using this amount of charge as an indicator of how much energy was deposited in the entire uranium-argon cell. But when particles move from one cryostat to the other they first pass through liquid argon between the modules and the cryostat walls that cannot be read out and then pass through the space in between the cryostats as well. The situation is made worse by the thick steel endplates of the modules which can cause the particles to start showering and deposit energy if they were not already. Special detector elements were constructed to detect such energy. The massless gap detectors are alternating sets of high voltage and grounded signal boards which were joined to the calorimeters module end plates inside the cryostat. In this way, particles showering on their way out of the calorimeter would deposit some amount of energy in the argon between these gaps and this could be read out. The space between the cryostats was filled with scintillators that made up the inter-cryostat detector (ICD). The ICD covers the MH ring and a portion of the $\mathrm{OH}$ as well. Rings of massless gaps were placed on the central calorimeter fine hadronic end plates as well as the endcap calorimeter middle hadronic and outer hadronic modules. Both these detectors were simulated and the 
results used for reconstructing jets. Large portions of jet energy can be deposited in these detectors if the jet points in these directions.

\section{Muon Chambers 5}

Three layers of muon chambers (one before the iron and two afterwards) make up the Wide-angle-muon-system which provides $2 \pi \phi$ coverage in the central region. Furthermore, special magnet iron and chambers provide extended coverage down to small angles (Small-angle-muon-system:SAMUS). Particle and heavy quark decay within the jet may produce muons which may not be contained by the calorimeter. Hence it is necessary to add such muons back into the jet. Currently this is not done in our present jet reconstruction.

1F. Nang and R. Partridge, Level 0 Detector Layout, D0 Note 998, D0 Experiment at Fermilab, Batavia, IL August 1990.

2 A. Clark et al, The Central Tracking Detectors for D0, CONF-8806235-8-mc (microfiche), (1988)

${ }^{3}$ Numerous references on the D0 Calorimeter exist. See reference 5

4P. Franzini, Nucl. Instrum. Meth., A263, 78 (1988)

5D0 Collaboration, An Experiment at DO to Study Antiproton-Proton Colllisions at 2 TeV: Design Report, Print-84-0306 (Fermilab) (1983) 


\section{Chapter 5: Theoretical Jet Definition}

Jets have traditionally been defined by the experimentalists as leading order perturbation theory predicts two final state partons which are well separated due to transverse momentum balancing. There is little ambiguity at this level; each quark is expected to manifest itself as a jet. In next-to-leading-order theory, the definition of a jet is not as well defined. We are concerned with the theoretical definitions of jets for a couple of reasons. First of all, a theoretical jet definition allows the calculation of theoretical distributions to which we can compare experimental results. Also, as we must simulate hard scattering events to test our reconstruction procedures, we need to identify the 'true' or 'theoretical' jets for comparison with our experimentally reconstructed jets. Finally, the theoretical jet definition will necessarily motivate our experimental jet definition and jet reconstruction algorithms.

We will discuss two theoretical jet definition types below. The first describes a definition that was used to calculate the single jet cross section to next to leading order in perturbation theory by Ellis, Kunszt and Soper. The second definition was used to identify theoretical jets from event generators, such as ISAJET, which approximate all orders of peturbation theory. The first definition must guide our experimental reconstruction of jets as it is our best guess at what is 'true' in real data. We will describe that definition in great detail. But the second definition is necessary for determining reconstruction biases and efficiencies. In this paper, the terms "theoretical jet" or "parton jet" or "particle jet" will always refer to one of these definitions and therefore does not necessarily refer to the 'spray of particles' which is the signature of an experimental jet. 
NLO Jet Definition According to Ellis et al. ${ }^{1}$

Perturbation theory simply predicts the final state partons of the event since the fragmentation process through which partons turn into a jet of hadrons is nonpeturbative At lowest order $Q C D$, there is simple parton elastic scattering and hence the final state consists of two final state partons which are well separated in space due to transverse momentum balancing. Since, it is experimentally easier to detect jets which are emitted at wide angles from the hadron beam line, the cross section is often described in terms of the transverse momentum $\left(P_{t}\right)$ and the rapidity (y) of the parton. $P_{t}$ is simply defined as $P \cdot \sin \theta$, where theta is defined as the angle between the final state parton and the incoming partons. The rapidity is defined formally as :

$$
y=\frac{1}{2} \ln \left(\frac{E+P \cos \theta}{E-P \cos \theta}\right)
$$

This is preferable as both quantities are Lorentz invariant. Hence the predicted quantity is: do/dPTdy.

However, NLO diagrams allow 2 or 3 parton final states. The third parton could be a gluon which can be emitted at small angles to one of the other partons and with small energies as well. Alternatively, it could be a gluon which splits into a quark-antiquark pair. Divergences as the energy of the third parton or the angle between it and the parent parton become small prevent the calculations of finite cross sections for a 'two parton' final state or a 'three parton' final state although the sum of the two is finite. These divergences can be removed by introducing an arbitrary parameter to distinguish the two states unambiguously. The common technique is to choose as this parameter a distance $R$ in $y-\phi$ space:

$$
R=\sqrt{\left(\Delta y^{2}+\Delta \phi^{2}\right)}
$$

and define a 'partonic jet' as the sum of all partons within a cone of radius $\mathrm{R}$. Figure 5-1 shows an example of this. 


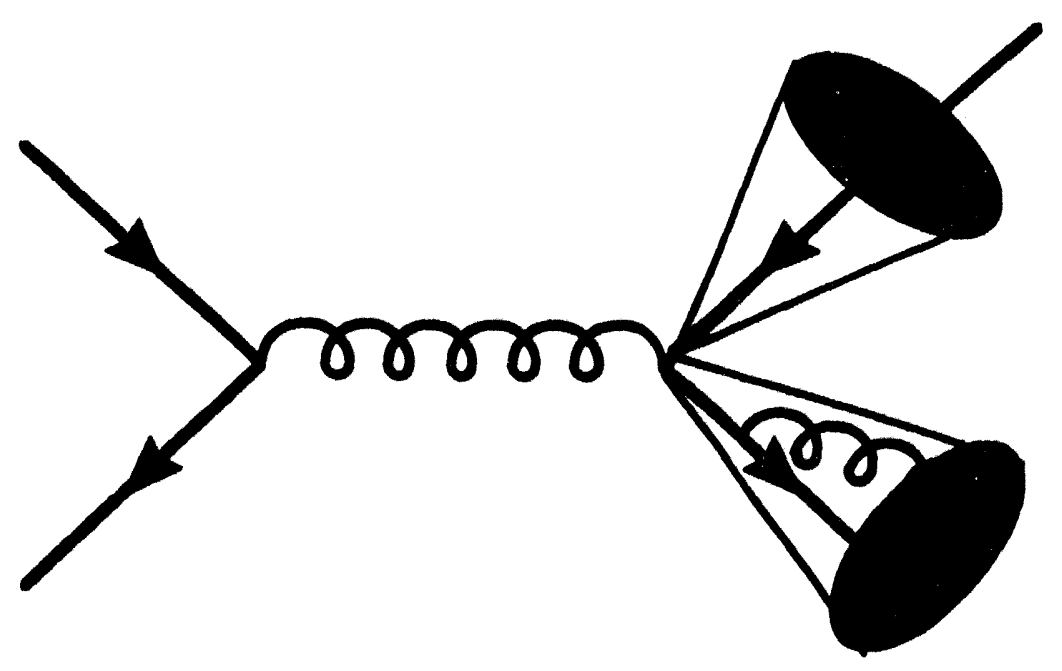

Figure 5-1: A cone definition of a jet allows unambiguous classification of jets from next-to-leading-order processes.

Thus for NLO QCD, the theory predicts $d \sigma / d P_{t} d y(R)$. This is the differential cross section for producing a partonic jet (using the cone definition of a jet with radius $\mathrm{R}$ ) with transverse momentum $\mathrm{Pt}$ with rapidity $\mathrm{y}$.

The next to leading order calculations of Ellis, Kunszt and Soper have been recently published. In addition, we have made use of a program written by them to predict inclusive jet cross sections. We will refer to results from this program as "Ellis results". As we intend to compare to their predictions, it is important to understand their definitions:

a) Each parton has a rapidity (y), azimuth direction $(\phi)$ and a transverse momentum $\mathrm{PT}_{\mathrm{T}}$

b) A cone algorithm is used to 'merge' a pair of partons if they meet the following criteria:

$$
\sqrt{\left(\left(y_{J}-y_{i}\right)^{2}+\left(\phi_{J}-\phi_{i}\right)^{2}\right)} \leq R ; i=1,2
$$

where $i=1,2$ refer to the partons under consideration and

$$
P T_{J}=\sum_{i=1,2} P T_{i} ; y_{J}=\frac{1}{P T_{J}} \sum_{i=1,2} y_{i} P T_{i} ; \phi_{J}=\frac{1}{P T_{J}} \sum_{i=1,2} \phi_{i} P T_{i}
$$

It then follows that two partons jets are merged into one jet if: 


$$
\sqrt{\left(\left(y_{1}-y_{2}\right)^{2}+\left(\phi_{1}-\phi_{2}\right)^{2}\right)} \leq \frac{P_{1}+P_{2}}{\max \left(\mathrm{PT}_{1}, \mathrm{PT}_{2}\right)} \times R
$$

If this case is true, the parton jets $P_{1}$ and $P_{2}$ are not included in the cross section, but only the merged jet which has transverse energy of $\mathrm{Pty}$ and a position of $\phi_{\mathrm{J}}$ and $\mathrm{y}_{\mathrm{J}}$.

Since the NLO predictions depend on the definition of the cone via the cone size $R$, the theory can be further tested by making measurements that depend on this dependence. Traditionally, the energy distribution within a jet was described only by fragmentation schemes; but the NLO predicts the widening of the previously pencil thin parton jets through gluon radiation and gluon splitting. Additionally, the dependence of the cross section itself has an explicit $R$ dependence as opposed to $L O$ theory. The NLO cross section is expected to vary as $A+B \ln R+C^{2}$. Figure 5-3 shows the predicted cross section dependence for jets with $P T=40$. $G e V$ at $y=0$, for both LO and NLO. The LO curve is completely flat, as expected, while the NLO curve rises as the cone size is increased and thus making it 'easier' for $40 \mathrm{GeV}$ to be contained in a cone. 


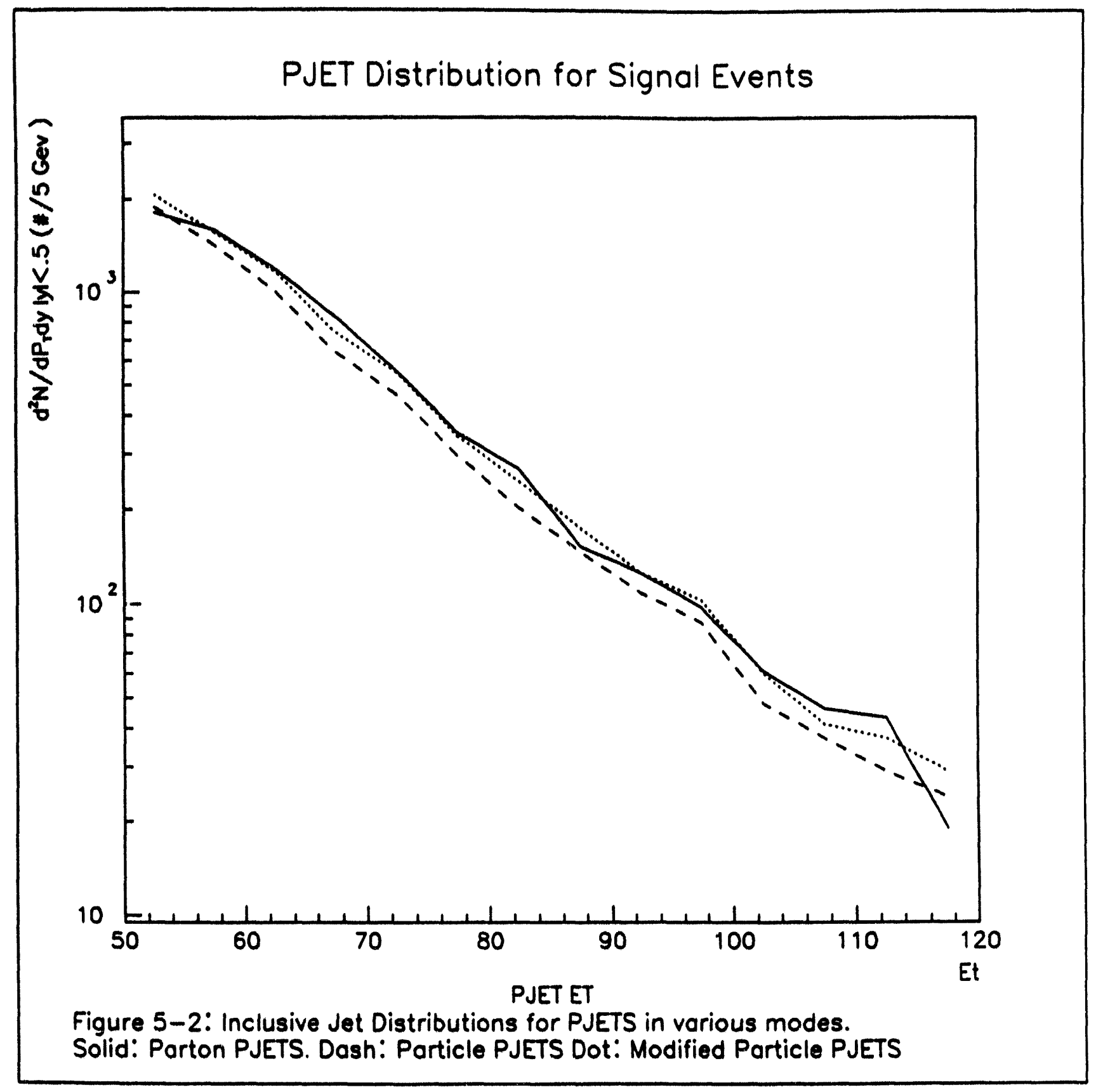




\section{Event Generator Jet Finder : PJETS}

As our event generators simulate jets physics to all orders of $\alpha_{\mathbf{s}}$, we need a theoretical definition for these as well. A software package was developed to provide this for the various event generators used in D0; it is called PJETS. This algorithm determines jets from either a) a list of the event's final state partons or b) a list of the events final state particles. We thus refer to them as parton jets or particle jets. The algorithm requires a cone size $(R)$ to be specified. The algorithm proceeds as follows: The list is Pt ordered Then the list is scanned from top to bottom. Each unused parton/particle on the list becomes the center of a jet. The rest of the list is then searched and any particles/partons within the given cone size is added into the jet. A new energy weighted jet center is found and the process can be repeated until the center is stable. But usually, the algorithm only does one repetition. The next particle/parton on the list (that has not been associated with a jet) is then used as a jet center and the process continues until all particles/partons have been considered. No jet merging or splitting is done. Figure 5-2 shows various (unnormalized) transverse energy distributions for the various PJET algorithms when a cone size of $R=.7$ is used. This sample was generated from ISAJET under the condition that one of the two initial generated partons had a rapidity value between $y=-.5$ and $y=.5$. Two of the curves correspond to the PJET algorithms described above. Note they do NOT fall on top of each other. The spectrum found when using the final state partons is systematically higher than the spectrum found when the final state particles are used. This might be explained if the fragmentation process caused partonic energy to leave the cone in the form of particles. More likely, fragmentation of the partons may widen the jet enough to make it seem like two jets instead of just one. In any case, the PJET definition is not completely compatible with the Ellis definition. This can be seen by examining the simple example of two equal energy partons separated by a distance of .8. The PJET parton algorithm described above will always see two parton jets if the cone size is $R=7$. But the Ellis definition would merge these two jets into one according to equation 5.5. Of course, PJETS was not meant to match the Ellis definition, but as a comparator for the reconstructed jet finder. The reasoning becomes circular when we then realize that we want the offline reconstruction to match the Ellis definition and the PJET definition to match the reconstruction definition.

We attempt to standardize our jet definitions by introducing and using a Modified PJET definition which takes normal parton or particle PJETs and applies the 
Ellis merging criteria (equation 5.5) to form merged jets when the criteria is satisfied. Figure 5-2 shows that the new modified particle PJET distribution in addition to the others. 


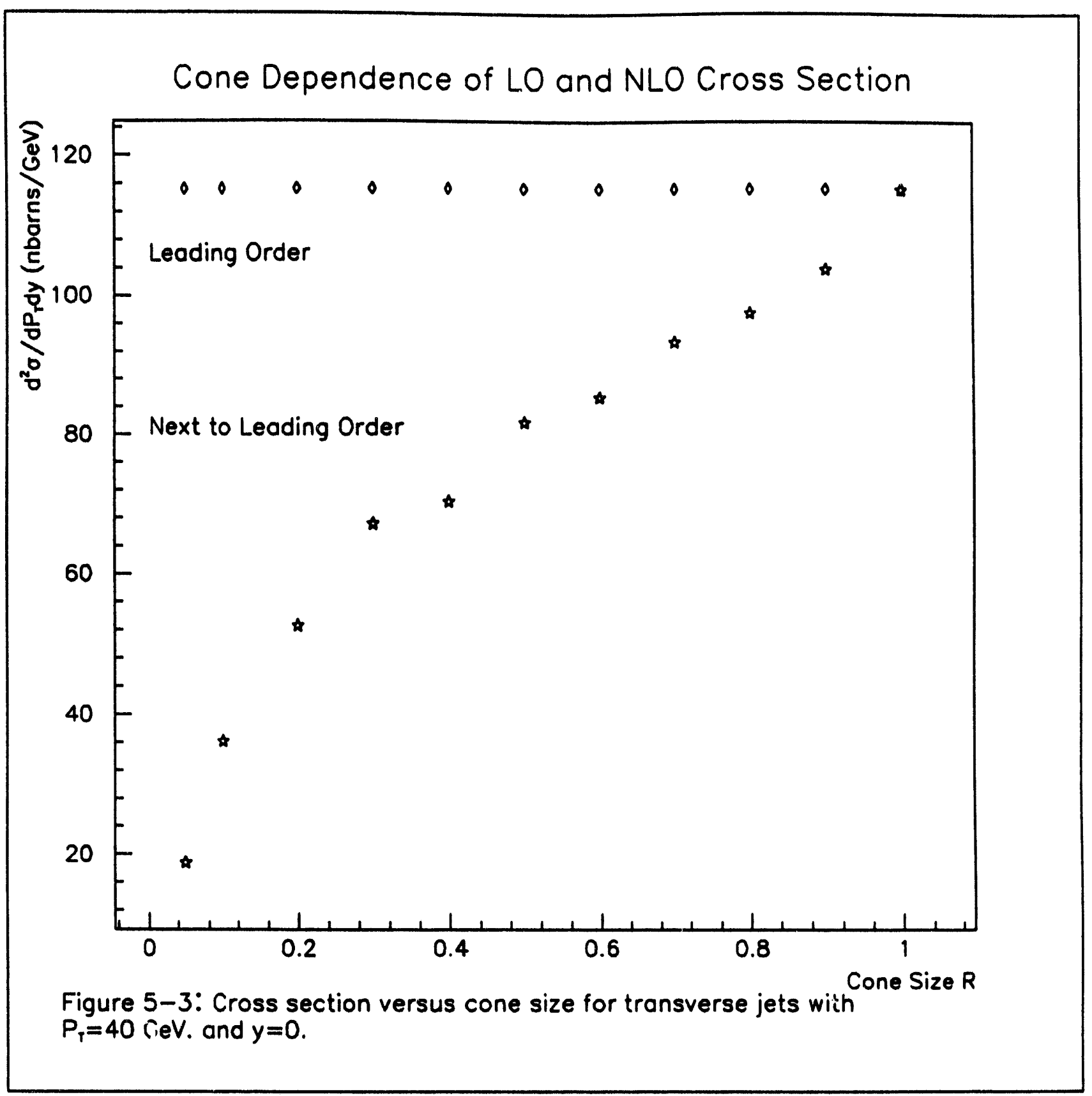




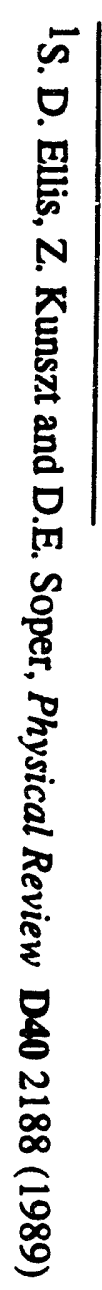




\section{Chapter 6: Do Jet Definition}

Since the scattered partons undergo fragmentation into a spray of particles and each particle than showers while in the calorimeter, a cone algorithm (or a similar energy clustering algorithm: is always necessary at the experimental level, even if it were known that only 2 partons were in the final state. This is because the partons fragment into particles before detection is possible. Figure 6-1 shows a histogram that relates the width of a parton jet versus the same jet after the partons have fragmented. This width is defined as a summation over all the partons or particles associated with the jet:

$$
\begin{gathered}
J_{e t_{\text {widh }}}=\sqrt{\eta_{\text {width }}^{2}+\phi_{\text {widh }}^{2}} \\
\text { where } \eta_{\text {wideth }}=\frac{1}{E_{\text {Jet }}} \sum E_{i}\left|\eta_{i}-\bar{\eta}\right| \text { and a similar definition holds for } \phi .
\end{gathered}
$$

This was obtained by looking at ISAJET parton jets and then tracing all the final state particles that had fragmented from any one of the partons in the jet. It can be seen that although the parton jet is always within a cone of $R=.4$, the fragmented jet can throw particles at wide angles from the original parton direction. Historically, the cone size is chosen to separate the energy that belonged to the parton from energy that was contributed by the two beam jets. This type of contamination is known as the 'underlying event'. Now that the NLO theoretical predictions include a cone size as well, it becomes important for the experimentalist not only to apply a cone to the jet, but to understand how much of the cone dependence is due to the underlying parton cone dependence and how much is due to the fragmentation of the partons and the showering of single particles in the detector. The process is further complicated by the assumption that the fragmented particles that make up the jet come only from the 
underlying parton. This cannot be true strictly as color conservation would require that the jet have color which it clearly cannot. Thus the identification of 'jet' energy with parton jet energv is flawed at some level. Fragmentation is not the only difficulty when using a cone algorithm. Since DO uses its calorimeters for jet measurement, each particle will shower in the calorimeter and spread its deposited energy over some area. Measurements at the $\mathrm{D} 0$ test beam ${ }^{1}$ indicates that the shower spread of one $50 \mathrm{GeV}$ pion is such that the shower is $95 \%$ contained by a circular region whose radius is $24 \mathrm{~cm}$ (the module being struck by the beam was the IH). In the central region, this area is contained by a cone of size $R=.2$. However, in the forward region, the cone size needed jumps to $R=.7$ due to the decreasing separation of rapidity lines for the region near the beamline. Hence, even a thin jet (or a single particle) will look broader in the calorimeter. 


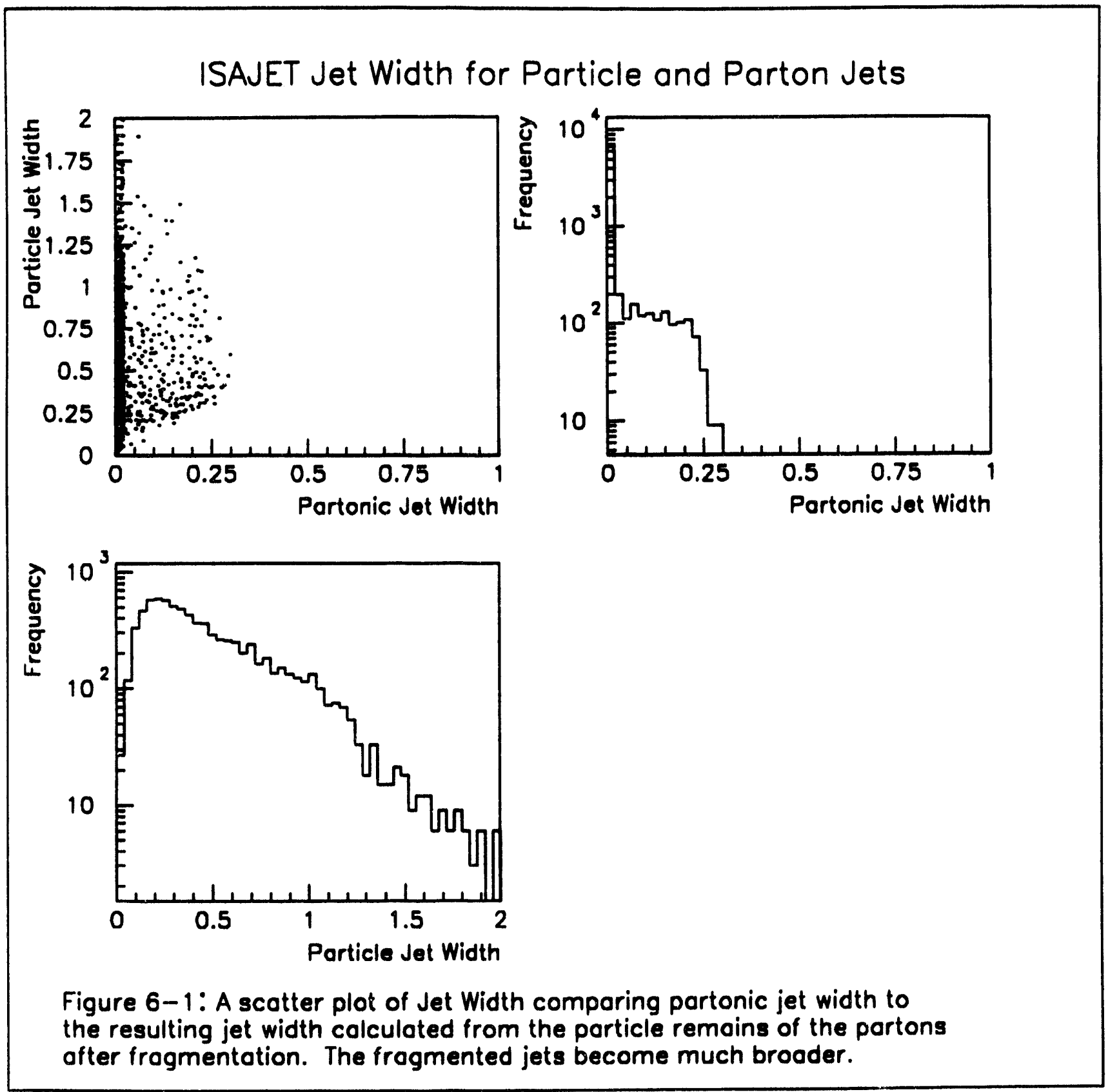


Still, the experimental jet definition is intended to mirror theoretical definitions in most respects. Some changes are required, however. Since the D0 calorimeter measures energy instead of momentum, transverse energy (ET) replaces PT in the algorithm. Further, the rapidity cannot be measured since that would require both momentum and energy knowledge. We make the small mass assumption that reduces rapidity to pseudo-rapidity which is defined simply in terms of $\theta$.

$$
\begin{aligned}
y & =\frac{1}{2} \ln \left(\frac{E+P \cos \theta}{E-P \cos \theta}\right) \quad \text { and } E \approx P \\
& \equiv \frac{1}{2} \ln \left(\frac{1+\cos \theta}{1-\cos \theta}\right) \\
\rightarrow & =\frac{1}{2} \ln \left(\cot ^{2} \frac{\theta}{2}\right)
\end{aligned}
$$

We refer to this quantity as pseudo-rapidity:

$$
\eta=-\ln \left(\tan \frac{\theta}{2}\right)
$$

Thus lines of constant angle from the beam line are lines of constant pseudorapidity. But these lines grow closer and closer together as the angle becomes close to 0 or 180 degrees.

Jets are reconstructed most accurately during offline analysis. The framework for jet reconstruction at $\mathrm{D} 0$ has long been in place and been tested extensively on a wide variety of both simulated $Q C D$ and rare physics events as well as simulated special events such as single pions and single electrons. Two algorithms are currently available. The first is a fixed cone algorithm which we will describe in more detail. The second is a nearest neighbor algorithm in which jets are formed by clustering cells following some prescription. Similar algorithms have been used by many other experiments. However, theorists are able to do calculations using only a fixed cone algorithm and so that is the one we concentrate on for now. We first give a description of the D0 jet reconstruction algorithm and then discuss how well it mirrors the Ellis definition. 
The steps of the algorithm are as follows 2 :

1)First the algorithm considers all the towers in the calorimeters. A tower is defined as one $\eta-\phi$ tower with all of its layers summed. Figure 6-2 shows an event as viewed in $\eta-\phi$ space broken up by readout towers. The towers are ordered by Et. There are $64 \phi$ towers and $74 \eta$ towers. This diagram shows two forward jets.

2) Preclusters are formed by sorting through the towers in order of Et. Only towers above some threshold (usually $2 \mathrm{GeV}$ ) are initially considered. The algorithm then looks at the nearest neighbors of these seed towers and joins them if they are above some smaller threshold (usually $1 \mathrm{GeV}$ ). Thus the calorimeter is divided up into preclusters and the rest of the lower Et towers. The average $\eta$ and $\phi$ of each cluster is calculated.

3) Each precluster is used as the center of a cone. All cells within a cone in $\eta-\phi$ space defined by the radius $R=\sqrt{\left(\Delta y^{2}+\Delta \phi^{2}\right)}$ are joined into the jet. A new $\eta-\phi$ average is calculated from the $\mathrm{E}_{\mathrm{T}}$ weighted $\mathrm{x}$ and $\mathrm{y}$ positions of the cluster and the procedure is repeated until the center of the cone is equal to the averaged $\eta-\phi$ of the jet to within some precision.

$$
\begin{aligned}
& E_{\mathrm{x}}=\sum_{\text {all colls }} \mathrm{E}_{\mathrm{x}_{\mathrm{i}}} \text { defined for } \mathrm{y} \text { and } \mathrm{z} \text { as well. } \\
& \phi_{\mathrm{J}}=\tan ^{-1}\left(\frac{\mathrm{E}_{\mathrm{y}}}{\mathrm{E}_{\mathrm{x}}}\right) \quad \theta_{\mathrm{J}}=\cos ^{-1}\left(\frac{\mathrm{E}_{\mathrm{z}}}{\sqrt{\mathrm{E}_{\mathrm{x}}^{2}+\mathrm{E}_{\mathrm{y}}^{2}+\mathrm{E}_{\mathrm{z}}^{2}}}\right) \quad \eta_{\mathrm{J}}=-\ln \left(\tan \left(\frac{\theta_{\mathrm{J}}}{2}\right)\right)
\end{aligned}
$$

4) The total $E_{T}$ of the jet is defined as the sum of the ET's of each cell.

$$
\mathrm{ET}=\sum_{\text {all cells }}^{\sum E T_{\mathrm{i}}}
$$

5) The list of jets is inspected for cases in which two jets share common cells. In this case the amount of energy shared is compared to the jet with the smaller ET. If the ratio of these numbers is above some splitting fraction (usually .5) the jets are merged. Otherwise, the energy is split evenly between the two jets. 3

6) Additional quantities for each jet are calculated. The electromagnetic fraction of the reconstructed jet is equal to the ratio of the jets energy which was found in any electromagnetic section of a calorimeter divided by the total energy. The second moments of the ET weighted $\eta$ and the $\phi$ distributions are calculated. The reconstructed jet width is defined simply as the sum of these two when added in quadrature. 


\section{Comparison to Ellis jet definition}

We will test our jet reconstruction abilities in the simulations by comparing the distribution of reconstructed jets to the distribution of simulated PJETS. This is because we are interested in recreating the input distribution and not necessarily the calculated distribution of the theorists as approximated in the NLO distribution. However, it is still important to verify that our reconstruction respects the Ellis definition as well. The reconstruction algorithm mirrors most of the Ellis definition with the exception of the merging criteria. Merging of jets is enforced in the reconstruction in two ways. The first is by changing the jet center until it matches the ET weighted center. Thus if the jet center starts in the direction of one of two closely spaced jets, the center will move in between the two and perhaps merge them both within the cone. The second, more explicit enforcement of jet merging is the splitting fraction which causes the merge of jets if they share too much energy. Although there are prescriptions for jet merging in the reconstruction algorithm as well as the Ellis algorithm, it is not clear that they are consistent with each other.

The following simple study was done to gauge the compatibility of the reconstruction and Ellis jet definitions with respect to jet merging. Since the Ellis merging criteria only applies to two partons at a time, we used ISAJET events in which we could find only two partons which were within $R=1.1$ of each other. The Ellis definition can then be applied to determine whether it is one jet or two. We further require that if the cone size is changed to .3 , that the partons satisfy the Ellis definition of 2 jets. By then comparing the number of reconstructed jets in each case, we can see how the experimental definition compares to the theoretical one. The only other requirements were that the PJET was within . 5 of $y=0$., that the $E_{T}$ was in the range from 75. to 140. GeV and that the smaller ET parton have an ET above 15. $\mathrm{GeV}$. Figure 6-3 shows the distribution of the Number of Ellis jets for various cone sizes. For larger cone sizes there is a spike at $\mathrm{N}=1$, but our selection of events forces this distribution to become a spike at $\mathrm{N}=2$ by the time the cone size is .3. Figure 6-4 shows the corresponding distributions for $\mathrm{dN}=$ Number of Ellis Jets - Number of Reconstructed Jets for a Splitting fraction of SPLF $=.5$. This distribution is usually at 0 (agreement) with shoulders at $\mathrm{dN}=1$ and -1 . Thus the disagreement goes both ways: sometimes the reconstruction sees an extra jet and sometimes it sees one less than the Ellis algorithm. But there is agreement from $70 \%$ to $100 \%$ depending on which cone size is used. This is encouraging considering we have chosen parton configurations where the disagreement should be at its worst. Figure 6-5 shows the 
distributions for SPLF $=.75$. Surprisingly, the curves are basically identical to the SPLF $=.5$ set; this implies the agreement between the reconstruction algorithm and the Ellis definition may be due to the recursive procedure for determining the jet axis and not to the value of SPLF. 


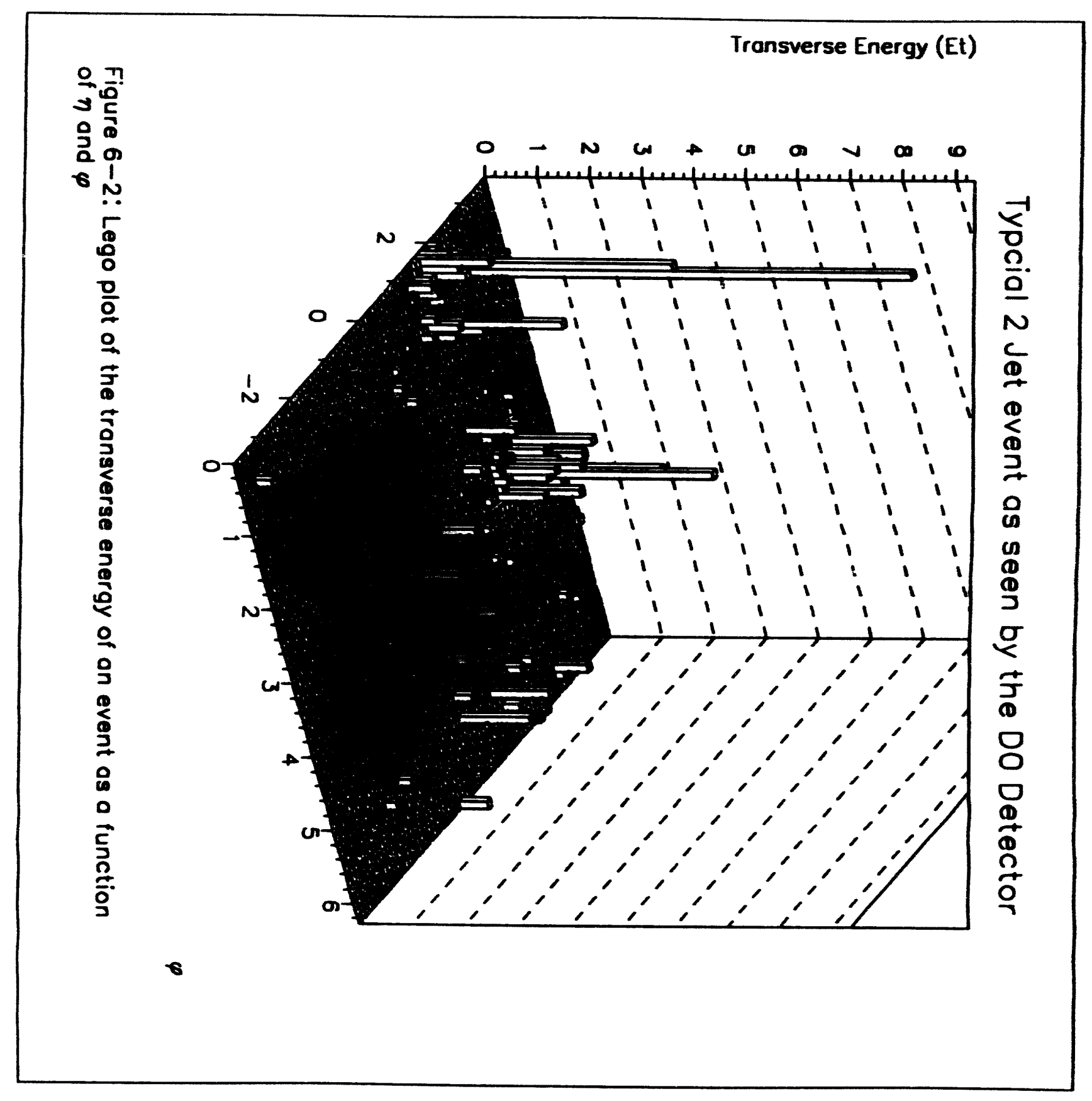




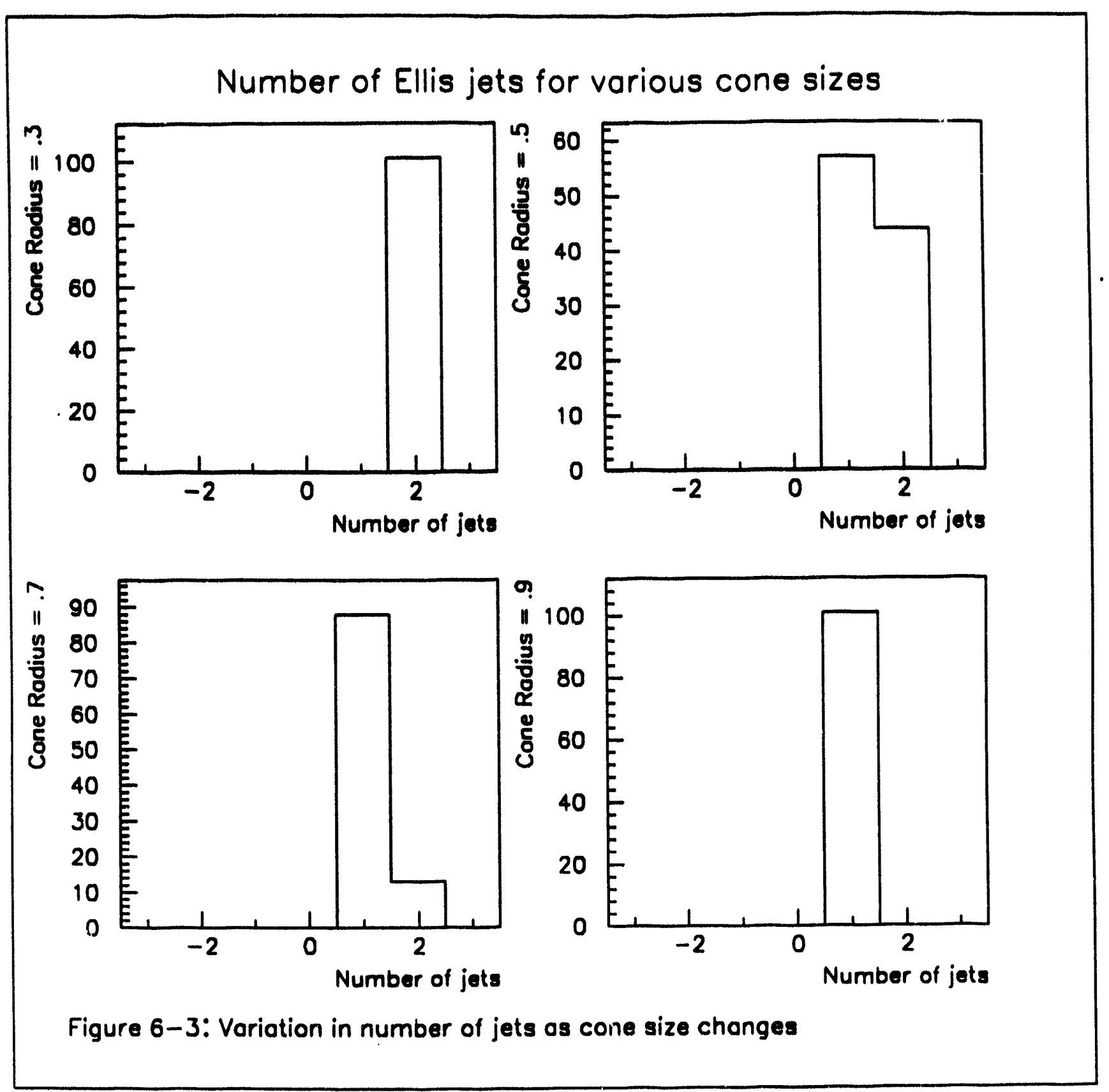




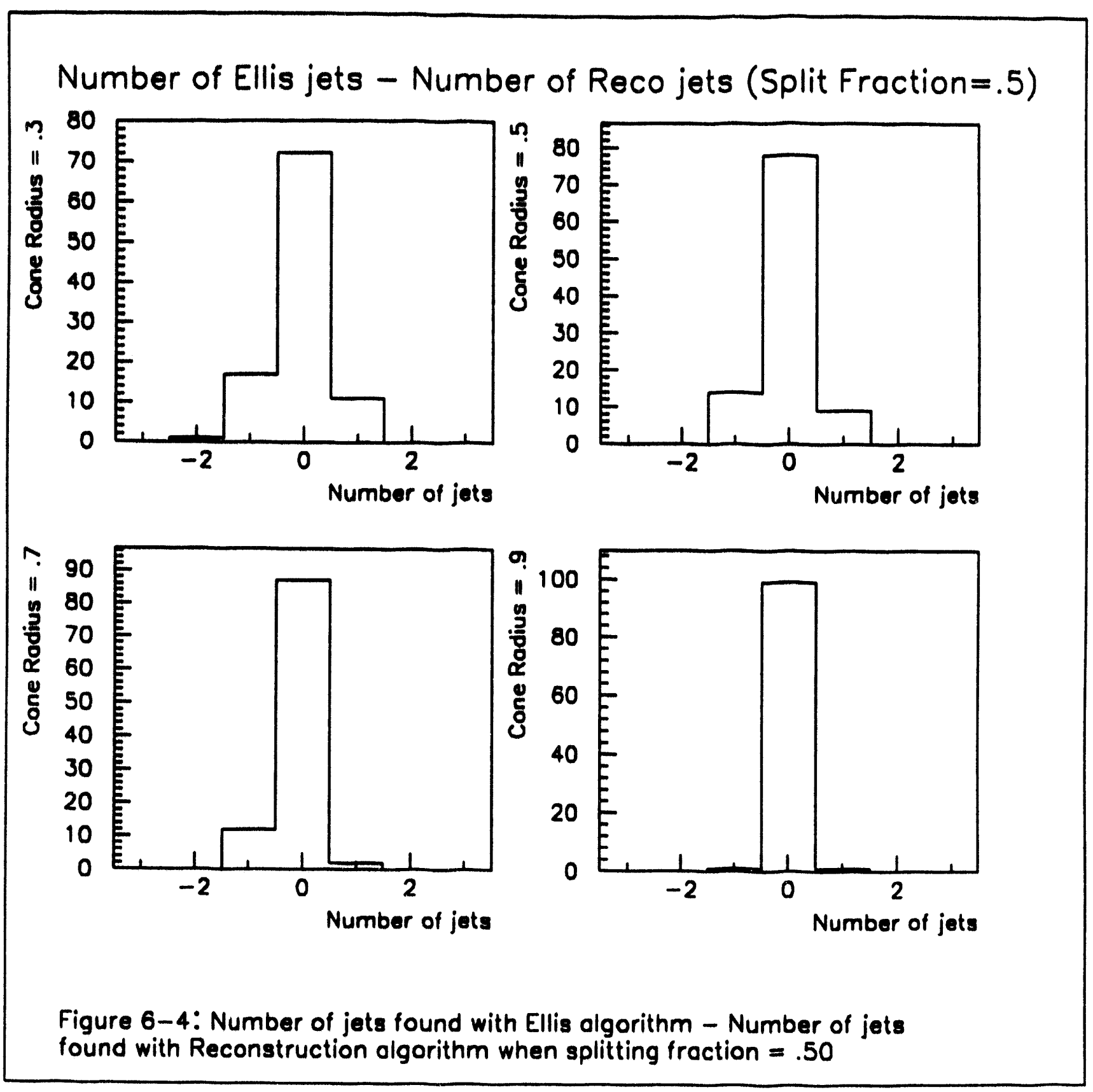




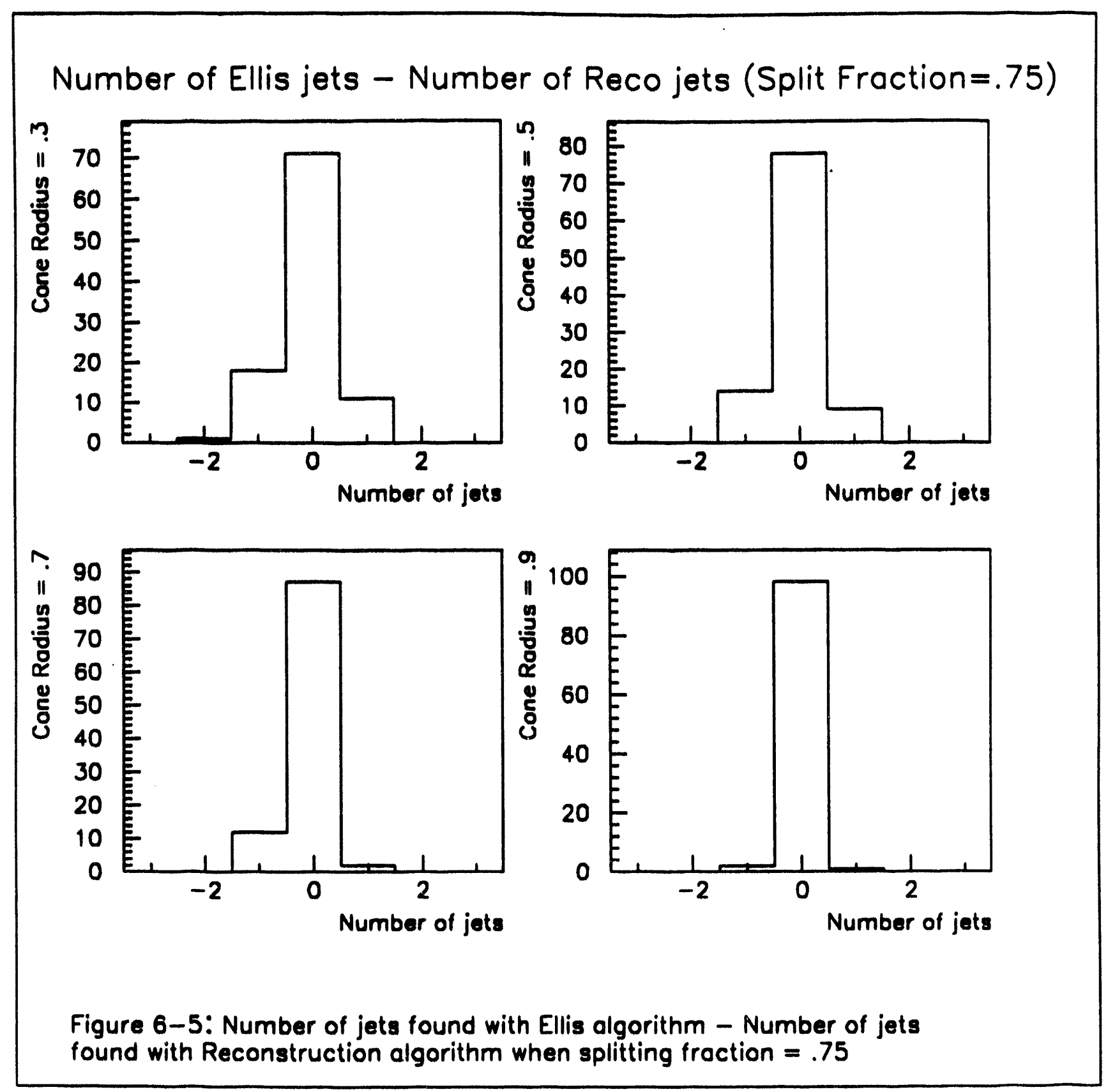


IS. J. Durston, Private communication.

${ }^{2}$ N. J. Hadley, Cone Algorithm for Jet Finding, D0 Note 904, D0 Experiment at Fermilab, Batavia, IL November 1989.

3N. J. Hadley, Splitting and Merging in Cajets., Internal memo. February 1991. 


\section{Chapter 7: Jet Reconstruction Results}

As mentioned at the beginning, we have used extensive computer simulations to determine the extent of DO's ability to reconstruct jets. Primarily we have used the ISAJET event generator to generate the final state partons and the hadrons produced by the fragmentation. To this extent we are biased to the ISAJET generation. To determine our jet reconstruction efficiency as well as the quality of the reconstructed jet characteristics, we must make comparisons between the generated jets and the reconstructed jets. In this chapter, a generated jet refers to a particle PJET; that is, a jet formed from the event generator's list of final state particles. We use the particle PJET instead of the parton PJET in order to isolate reconstruction biases and problems without the complications of fragmentation. A reconstructed jet refers to a jet found by the $\mathrm{D} 0$ reconstruction algorithm. In both generated and reconstructed algorithms, the cone size was $\mathrm{R}=.7$ and the reconstruction jet splitting parameter was .5 (jets that shared more than $50 \%$ of the least energetic jet are merged). Additionally, reconstructed jets that had been split or merged were considered only in the efficiency studies. We determine the reconstruction efficiency by searching for a reconstructed jet match for each generated jet. The requirement for a match was simply that a reconstructed jet was located within a distance of .7 in $\eta-\phi$ space of the generated jet. If more than one jet satisfied this requirement, then the distance requirement was changed to .5 . If more than one still satisfied, the reconstructed jet nearest in energy was taken. The matching between generated and reconstructed jets is 1-1. We will see below that these matching requirements are not overly strict. We will determine the quality of our reconstructed jet characteristics by comparing a generated jet to its reconstructed match. We define two quantities for such comparison which will be used here and in the following chapters: 


$$
\begin{aligned}
& R(\Psi)=\frac{\Psi_{\text {Reconstructed }}}{\Psi_{\text {Generated }}} \\
& \Delta_{\Psi}=\Psi_{\text {Generated }}-\Psi_{\text {Reconstructed }}
\end{aligned}
$$

where $\Psi$ can refer to any jet quantity (e.g. energy (E), transverse energy (ET), phi direction $(\phi)$ etc.).

Figure 7-1 shows a histogram of the quantity $\Delta \phi$. This distribution peaks at zero and has a width of .03 which corresponds to $1 / 3$ the size of a readout tower. Also shown is a plot of this quantity versus $\eta$ and a mild bias is apparent for large $\eta$. Figure 7-1 shows the same plots for $\Delta \eta$. The width of the distribution is about the same as in phi, but now there is a larger $\eta$ bias which results in an $\eta_{\text {reconstructed that }}$ is prejudiced away from the beam line. This is attributed partly to the finite shower size of particles within the jet ${ }^{1}$, the decreasing size of the readout towers as $\eta$ increases and partly to the asymmetric size of the cone in the forward region. This will be discussed in the calibration section. Even still, the magnitude of the bias is at worse one-fifth of a readout tower. The small widths of these distributions verifies that the matching requirements used were generous. The generated jets in these histograms had a ET of approximately $40 \mathrm{GeV}$. 
Reconstructed Jet $\eta$ and $\varphi$

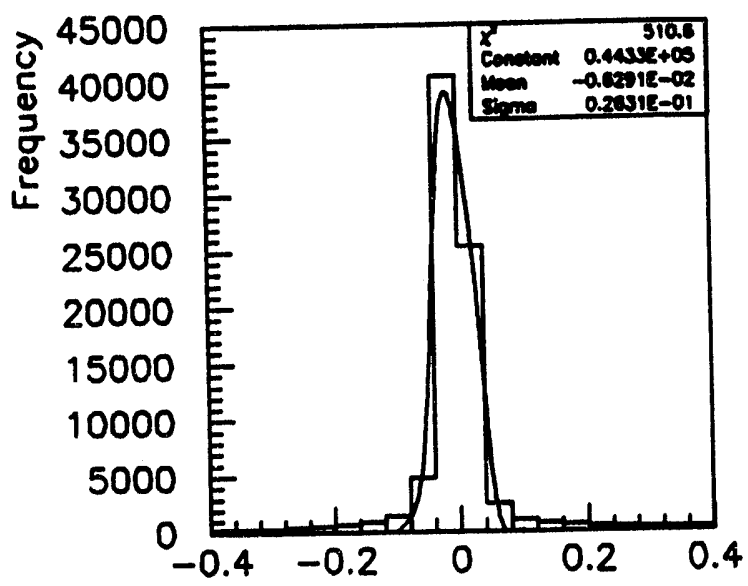

Generated $\Phi-$ Reconstructed $\Phi$
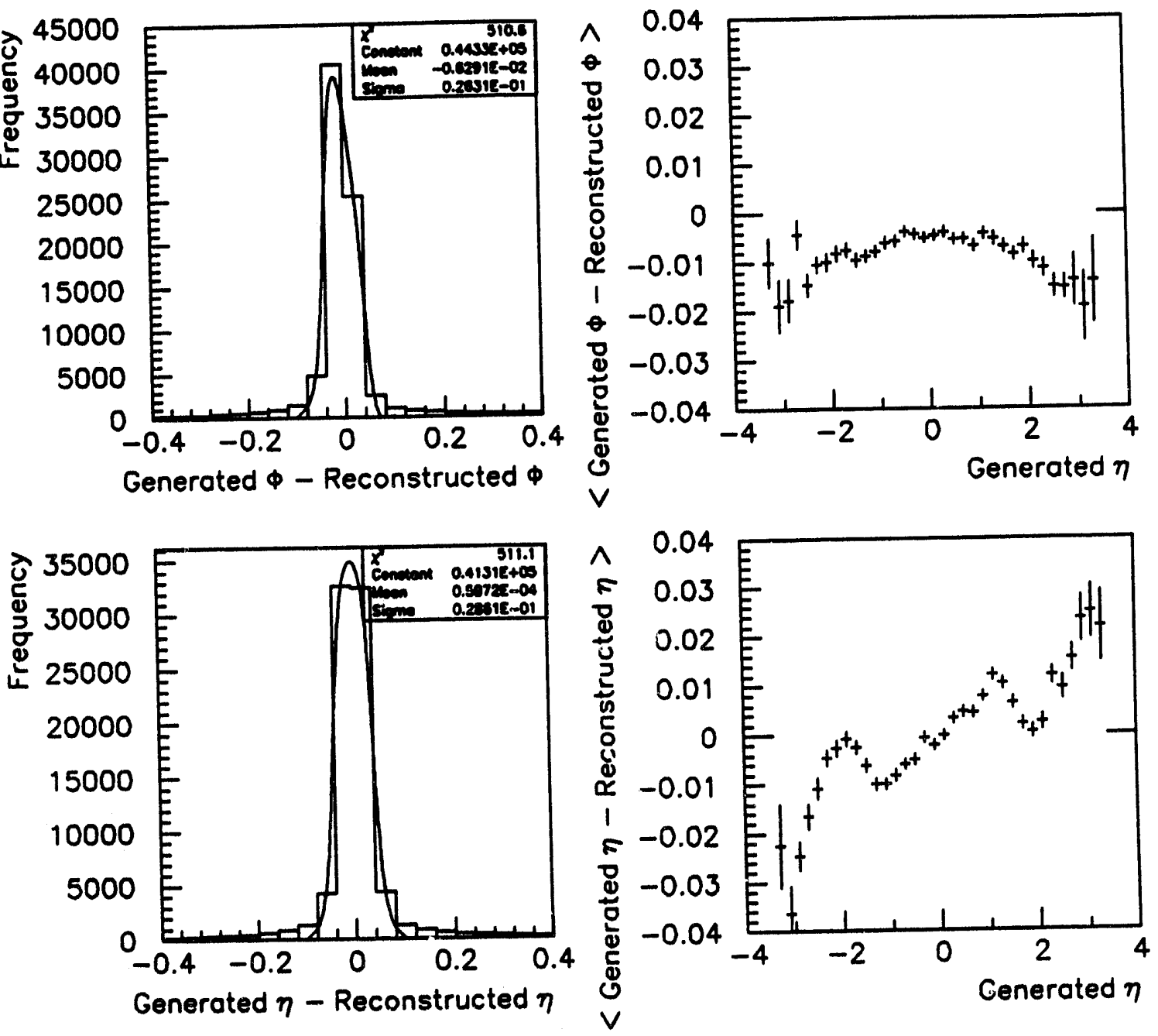

Figure 7-1: $\phi$ and $\eta$ reconstruction biases for Jets with $E_{T}$ between 35 and $55 \mathrm{Gev}$. A clear $\eta$ dependant bias is shown in the $\eta$ reconstruction. 
Figure 7-2 shows the distribution of $R(E)$ and $R(E T)$. This is a complicated plot which will be discussed further in the calibration chapter ${ }^{2}$. One evident feature is the over-estimation of ET compared to E. This is partly due to the $\eta$ bias described above. The $R(E)$ curve shows a systematic jet energy loss of approximately $20 \%$. The generated jets in this histogram all had at least $40 \mathrm{GeV}$ in transverse energy. Note that generated jets with the same $\mathrm{ET}_{\mathrm{T}}$ have larger energies for larger pseudorapidities than at $\eta-0$ where they are the same.

The predicted mismeasurements are due to detector systematics which we can try to correct and we will discuss that in the next section. Another question that must be addressed when talking about jet reconstruction, is that of reconstruction efficiency. We define jet efficiency as the percentage of generated jets for which we found a reconstructed match. Finding a match can be difficult when jets are nearby. Figure 7-3 shows histograms of both generated jets and those generated jets for which a reconstructed match was found. The generated jets were selected from the region in which the reconstruction begins to be inefficient. The curves are nearly identical above $\mathrm{ET}_{\mathrm{T}}=16 \mathrm{GeV}$ which implies complete efficiency. Jets below this energy are more difficult to reconstruct because they tend to be jets that are associated with nearby jets, or broad jets whose most energetic tower may not exceed the $2 \mathrm{GeV}$ threshold required to initiate a reconstructed jet. 


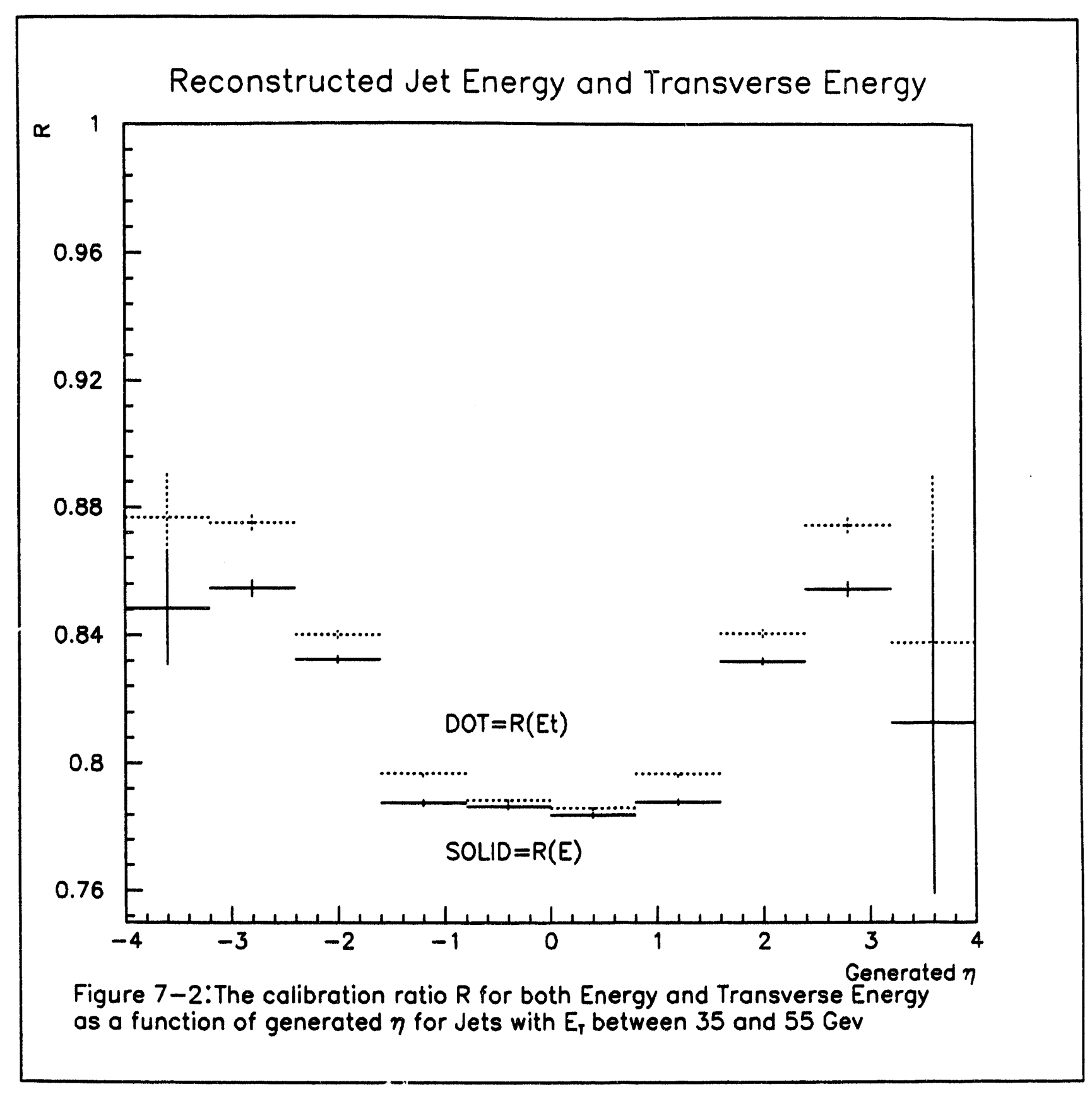




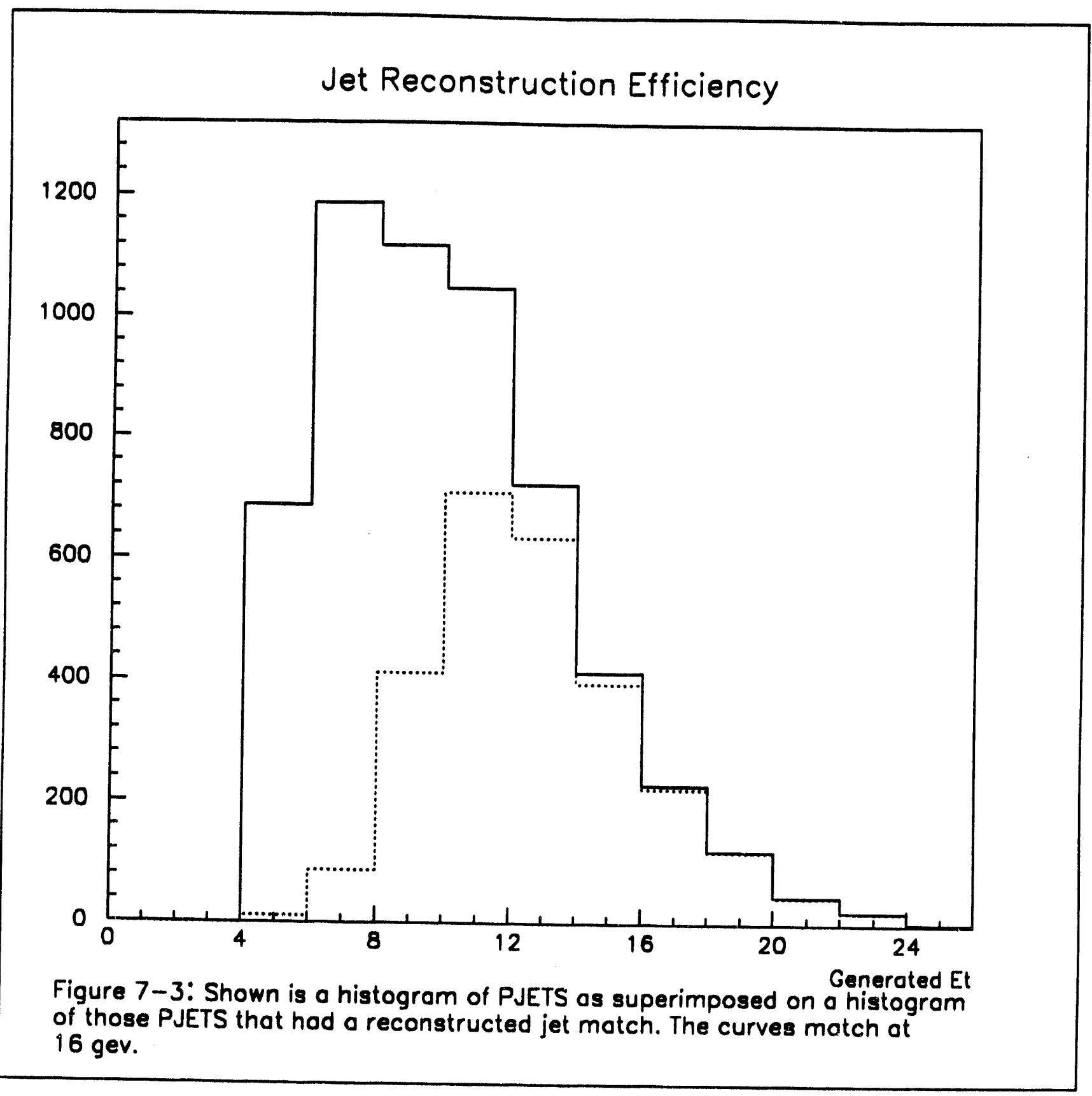




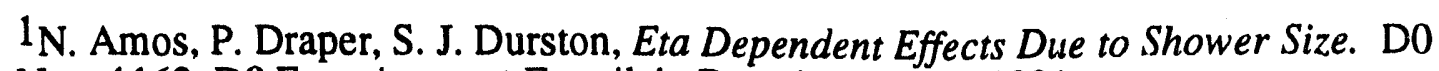
Note 1162, D0 Experiment at Fermilab, Batavia, IL, July 1991.

2S. J. Durston, Energy Calibration and Systematics, D0 Note 1202. D0 Experiment at Fermilab, Batavia, IL. Septermber, 1991. 


\section{Chapter 8: Jet Calibration}

The need for ET calibration is obvious from the previous section which shows that the reconstruction underestimates the $\mathrm{ET}$ of the average jet by about $16-20 \%$. There is also a reconstructed $\eta$ bias, but only for the very forward regions. We will first detail some of the causes of these mismeasurements. Then we will suggest various 'jet calibration' schemes for correcting the reconstructed ET. Since there are always differences between the behavior of a computer simulation and the real detector, we will discuss especially calibration methods that use as little simulation specific knowledge as possible. Such a scheme would be ideal for jet calibration in the first few months of DO datataking.

\section{Causes of reconstruction $\mathrm{ET}_{\mathrm{T}}$ mismeasurement in the reconstruction}

1) Cracks and dead regions in the calorimeter. Particles may deposit energy in the azimuthal cracks between the modules, the area between cryostats or other uninstrumented regions where there is no 'live' calorimetry to measure the energy deposit. Also, particles that are produced too far forward may miss the calorimeter altogether or deposit energy in the central detector. Although not included in our calorimeter simulation, the real calorimeters have many other dead areas such as notches in the signal boards and absorber plates and spacers which keep the cell spacing regular.

2) el $\pi$ The calorimeter does not respond to electrons and photons in the same way as it responds to pions and other hadrons. This effect is often referred to as the $e / \pi$ ratio. This number is determined by injecting pions and electrons of the same energy separately into a region of the calorimeter and measuring the response. This 
was done in the D0 Testbeam using Fermilab's fixed target beam. The $e / \pi$ is the ratio of the electron response to the pion response. This is done for several different energies and an e/ $\pi$ curve can then be produced. Figure 8-1 shows such a curve as determined from monoenergetic electrons and pions that are evaluated by the detector simulation. Typically the e/ $\pi$ ratio is greater than 1 . This implies that the detector has a greater response to electrons than to pions. Since it is very difficult to identify the type of particles captured in the detector, such curves are more useful when one assumes that photons will respond as electrons do, and that hadrons respond approximately the way pions do. e/ $\pi$ is important when measuring jets as jets can be either be very hadronic or very electromagnetic; on the average $33 \%$ of the jet's energy enters the calorimeter as photons. Note that knowing the $e / \pi$ is not directly applicable for calibration unless you can determine whether the energy in each cell was deposited by a electron/photon or a pion/hadron.

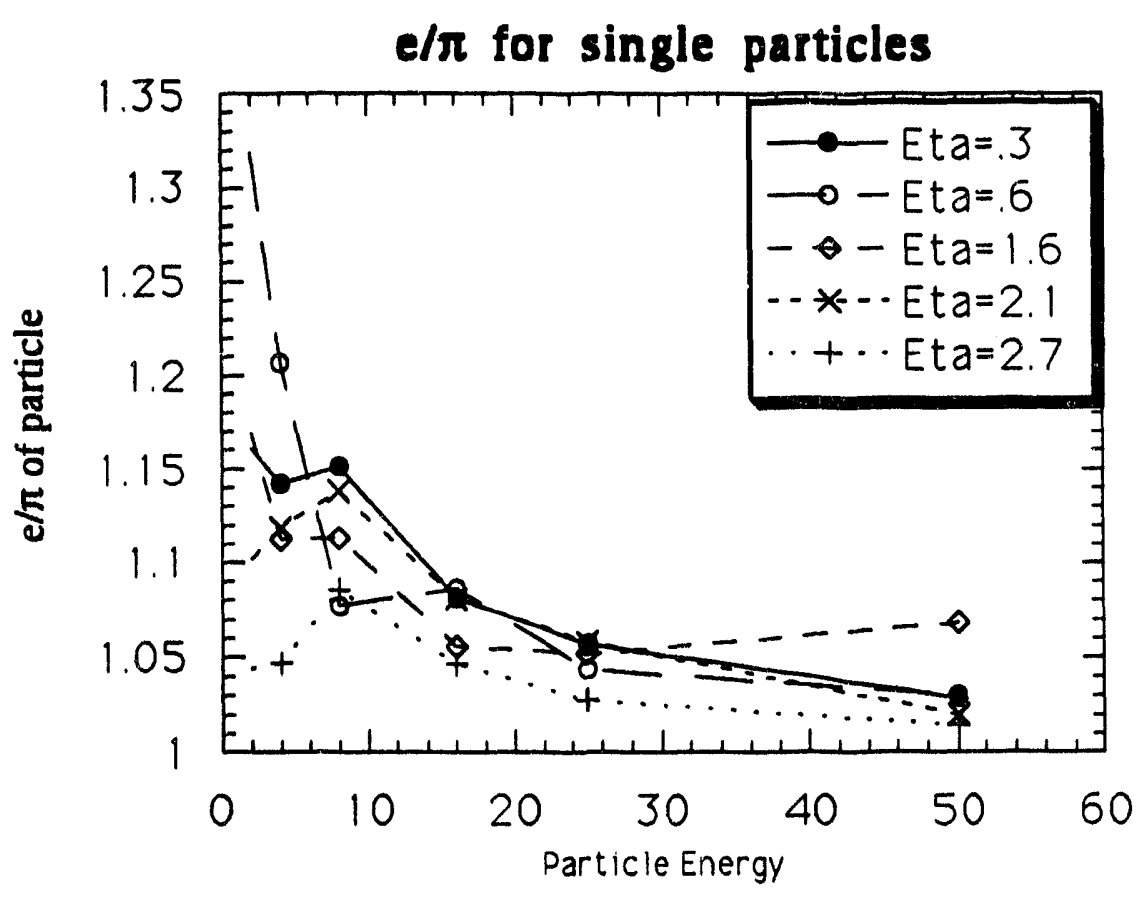

Figure 8-1: e/ $\pi$ as a function of particle energy and detector $\eta$

3) Nonlinearity. The calorimeter responds differently to identical particles at different energies. 1 This is particularly a problem since jets are made of a variety of particles at various energies. Consider figure 8-2 which plots the fraction of total jet energy carried as a function of the fractional jet energy of the particles that carry it. It 
is easier to interpret figure 8-2 if an example is given. The value of the first bin is .35 . The first bin has a width of .05 and holds values from 0.0 to 0.05 . The interpretation is that, on the average, $35 \%$ of a jets energy is carried by particles that have less than $5 \%$ of the total jet energy. This information is taken from the ISAJET event simulator and hence may be biased to the fragmentation scheme it uses. The first two bins contain almost $56 \%$ of the total jet energy hence we say: on the average, $50 \%$ of the jets energy is carried by particles with less than $10 \%$ of the total jet energy. Figure 83 shows the distribution of the first bin. We can see that some jets have as much as $80 \%$ of their energy carried by particles with less than $3 \%$ of the total jet energy. This idea is extremely important in jet calibration as one naively might try to treat a 200 $\mathrm{GeV}$ jet like a $200 \mathrm{GeV}$ particle. Instead, figure 8-2 implies that it is more accurately described by many $5 \mathrm{GeV}$ particles and a few energetic ones. If the calorimeter response is exactly linear, then the energy distribution of the particles within a jet does not matter. Figures 8-4 and 8-5 show the fractional responses $R(E), R(E T)$ for simulated single electrons and pions injected into the calorimeter at various angular regions but scattered over all $\phi$. It is important to understand how this was done. As single particle events were used and there was no noise in the simulation, all of the energy deposit was due to the electron or pion. Thus all the energy was added up and attributed to the single particle. The figures show consistently better response for higher energies. In order to calibrate, we would ideally like to determine $R\left(E_{T}\right)$, for example and multiply $1 / R\left(E_{T}\right)$ by Etreconstructed to determine the 'real' jet transverse energy. A non-linear curve implies that one must first group the energy that was deposited by each particle and then correct using the appropriate $R(E)$ value.
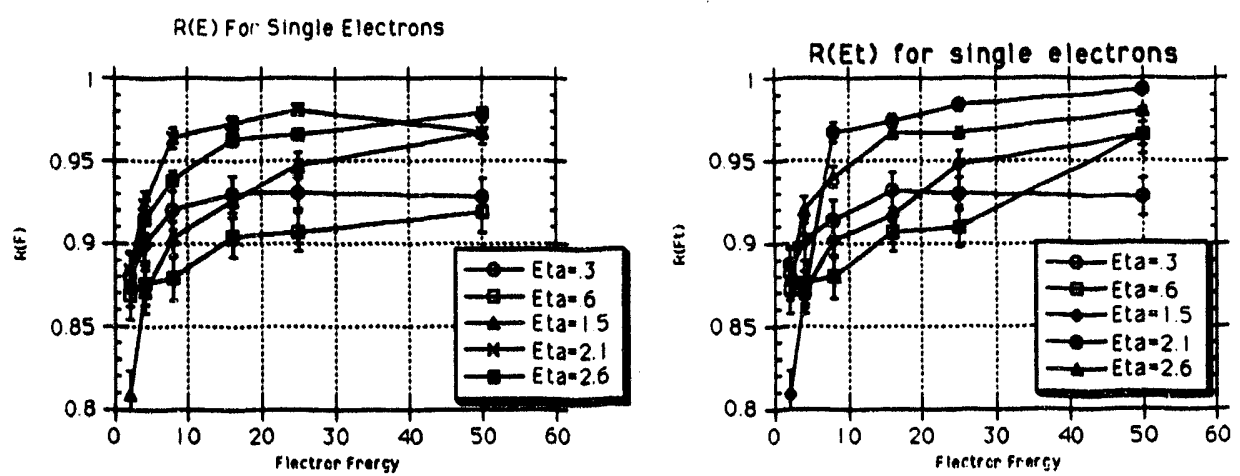

Figure 8-4: $R(E)$ and $R(E T)$ for single electrons as a function of electron energy and detector psuedorapidity. 

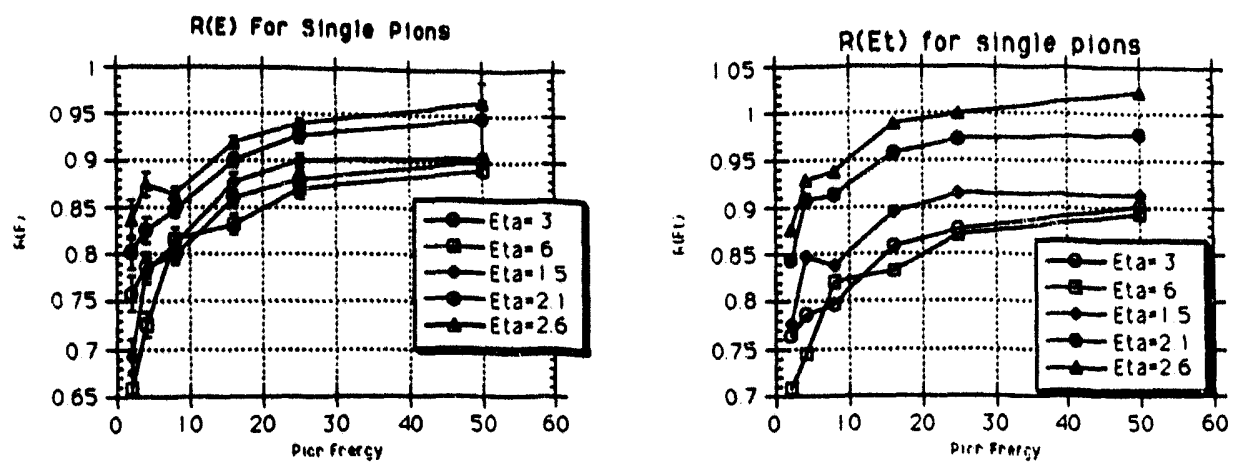

Figure 8-5: $R(E)$ and $R(E t)$ for single pions at various energy and psuedorapidity 
Jets with Energy from 200 to $300 \mathrm{Gev}$

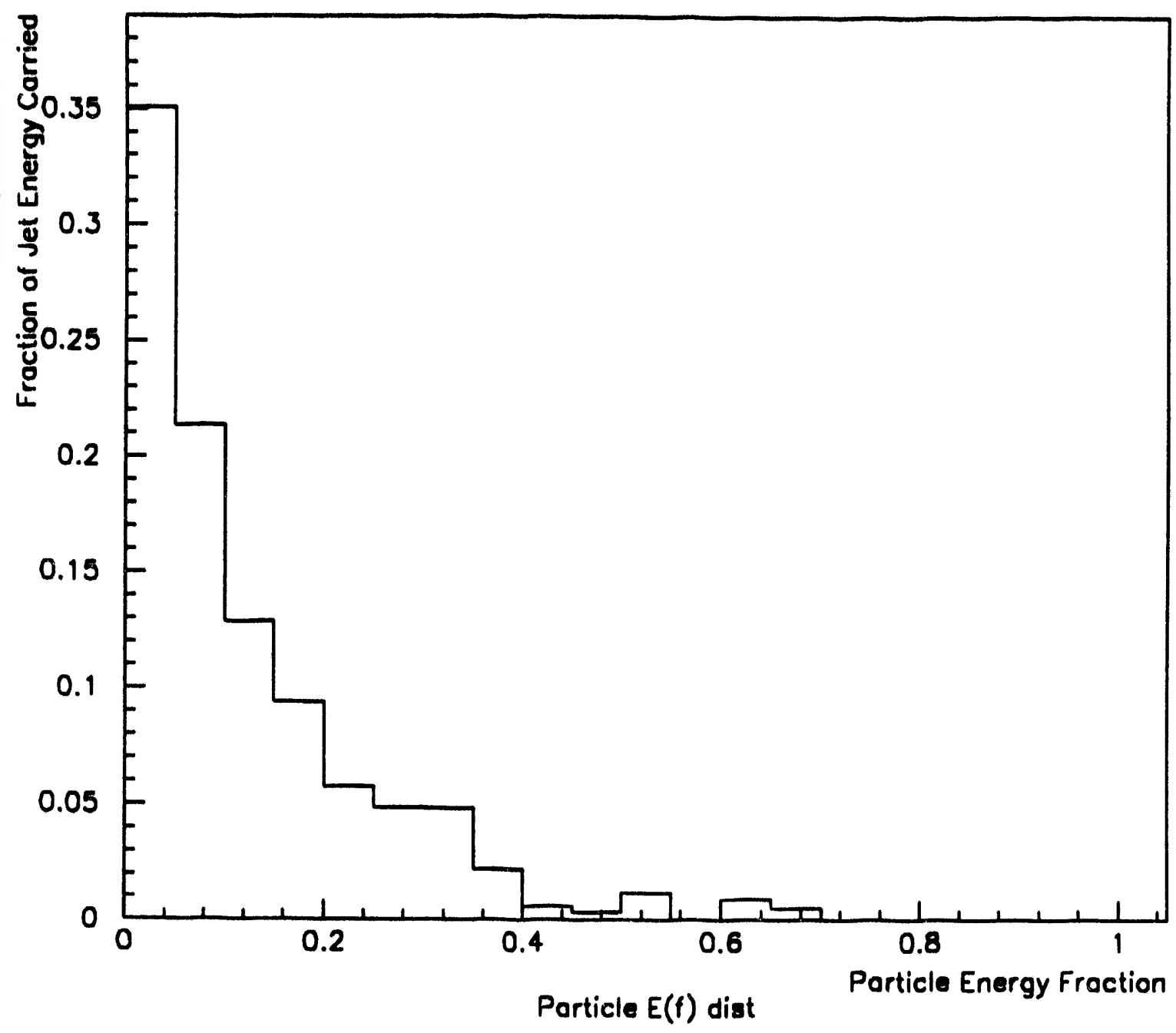

Figure 8-2 shows (for example) that, on the average, $35 \%$ of a Jets Energy is comprised of particles that each carry less than $5 \%$ of the total jet energy. 
I

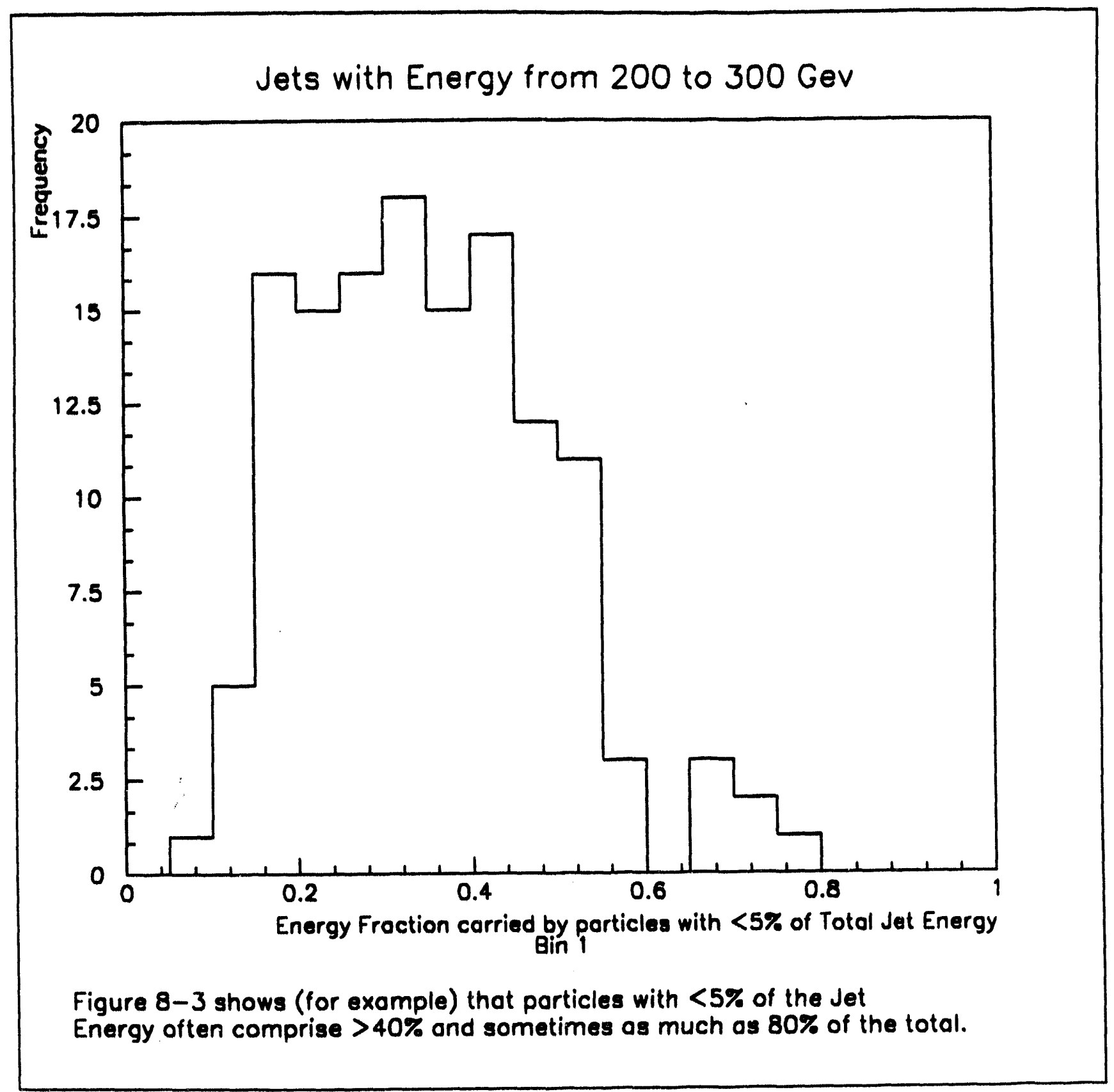


4) Out of cone showering As discussed in the section on the theoretical definition of a jet, the cone algorithm is used to define the energy of a jet. This cone is meant to be applied to the final state partons of the event. Since the calculation does not include the fragmentation of the partons, the loss of energy due to particles leaving the cone is not included. Experimentalists have traditionally attempted to correct both for this effect and the compensating effect of particles entering the cone from other jets or from the beam jets. We refer to these type of effects as "Out of cone" gains or losses. Nominally, we have removed this effect in our studies of jet reconstruction by comparing the reconstructed jet to a generated jet which is formed from the final state particles instead of the final state partons. However, particles within the cone start to shower and the size of the shower can be wide. Thus particles near the edge of the cone may deposit energy outside of the cone. Our reconstruction algorithm compensates to some degree by clustering groups of energy and using the center of such clusters to decide whether inclusion into the jet cone is necessary. If there were an effect due to energy spilling out of the cone, (see figure 8-7), it might show up first in the forward regions where the lines of constant rapidity grow close together. Figures 8-4 and 8-5 show no such effect for single particles because they were made using all the energy in the event and no cone was applied. As discussed in chapter 6 , the cone size needed to contain $95 \%$ of a $50 \mathrm{GeV}$ pion seen at the D0 test beam would be $R=.2$ in the central region but would be $R=.7$ in the forward region. The simulated single particles show this type of behavior as well. Figure 8-20 shows the difference in response for $50 \mathrm{GeV}$ and $2 \mathrm{GeV}$ pions in the case where all the energy in the detector is used versus the case where only the energy within a cone of $\mathrm{R}=.7$ is used. The former is from the curves in figure $8-5$. We see that $50 \mathrm{GeV}$ pions have nearly all of the detectable energy contained in a cone of $R=.7$ except in the very forward regions where $1-2 \%$ of the energy is lost outside the cone. The $2 \mathrm{GeV}$ curve shows a 3\% loss even in the central region and this becomes $11 \%$ in the very forward region. We expect the same effect for jets and indeed, figure 7-2 shows a strong drop in response for $\eta>3.0$. We might expect that jet response would be dependent on the cone size used. Figure 8-6 shows the average $R(E)$ versus $\eta$ for $50 \mathrm{GeV} \mathrm{ET}_{\mathrm{T}}$ jets with various cone sizes. (The generated jet cone size and the reconstructed jet cone size are kept the same.) One can see that for a cone size of .9, the $R(E)$ curve behaves better in the forward region as the single particle curves might predict. But smaller cone sizes drop in response by as much as $15 \%$ compared to the central region even when they are far from the beam pipe. 
It seems clear that out of cone losses will be very important in jet reconstruction in the forward regions and with small cone sizes.

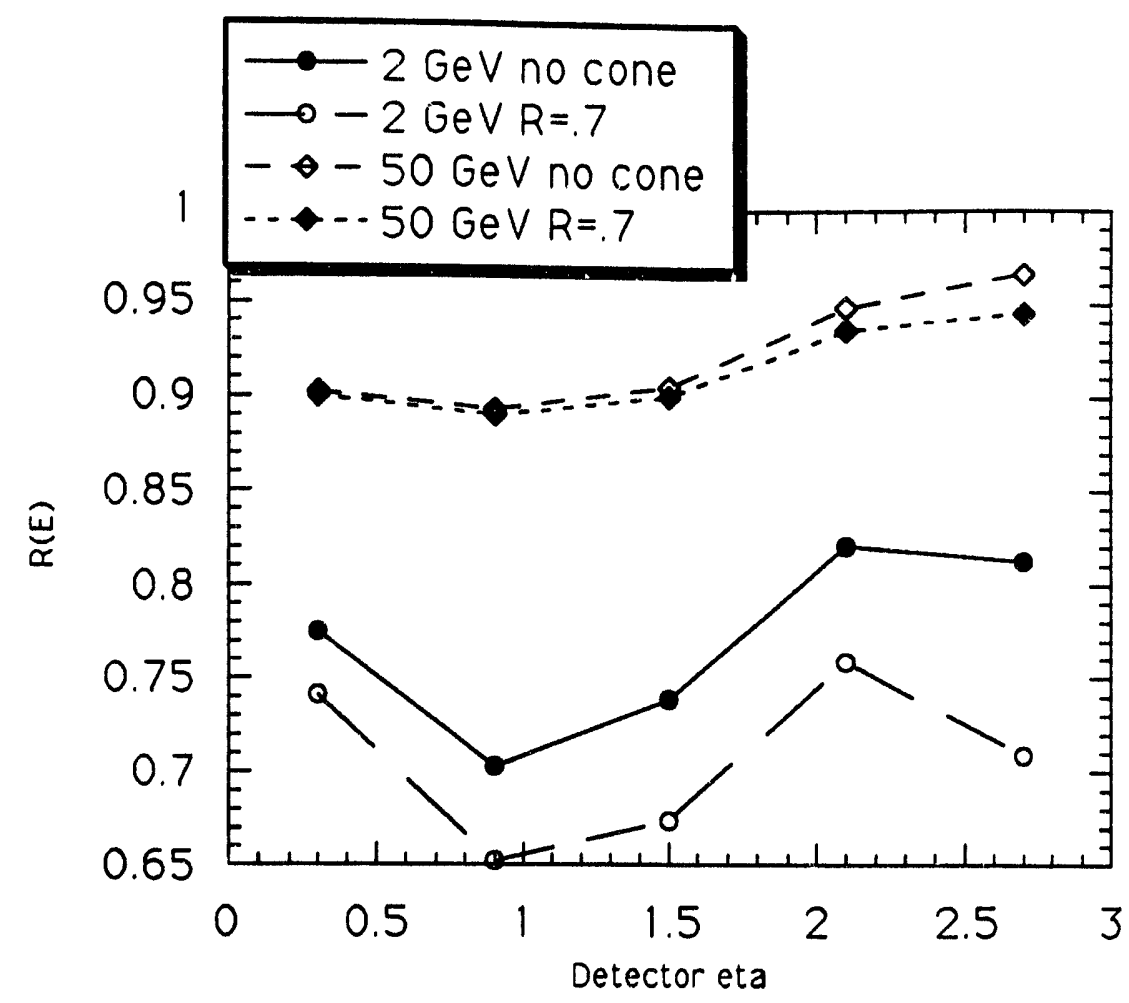

Figure 8-20: The respor with no cone (all energy . is is shown both with a cone of $\mathrm{R}-.7$ and used). 
$R(E)$ versus $\eta$ for different cone sizes

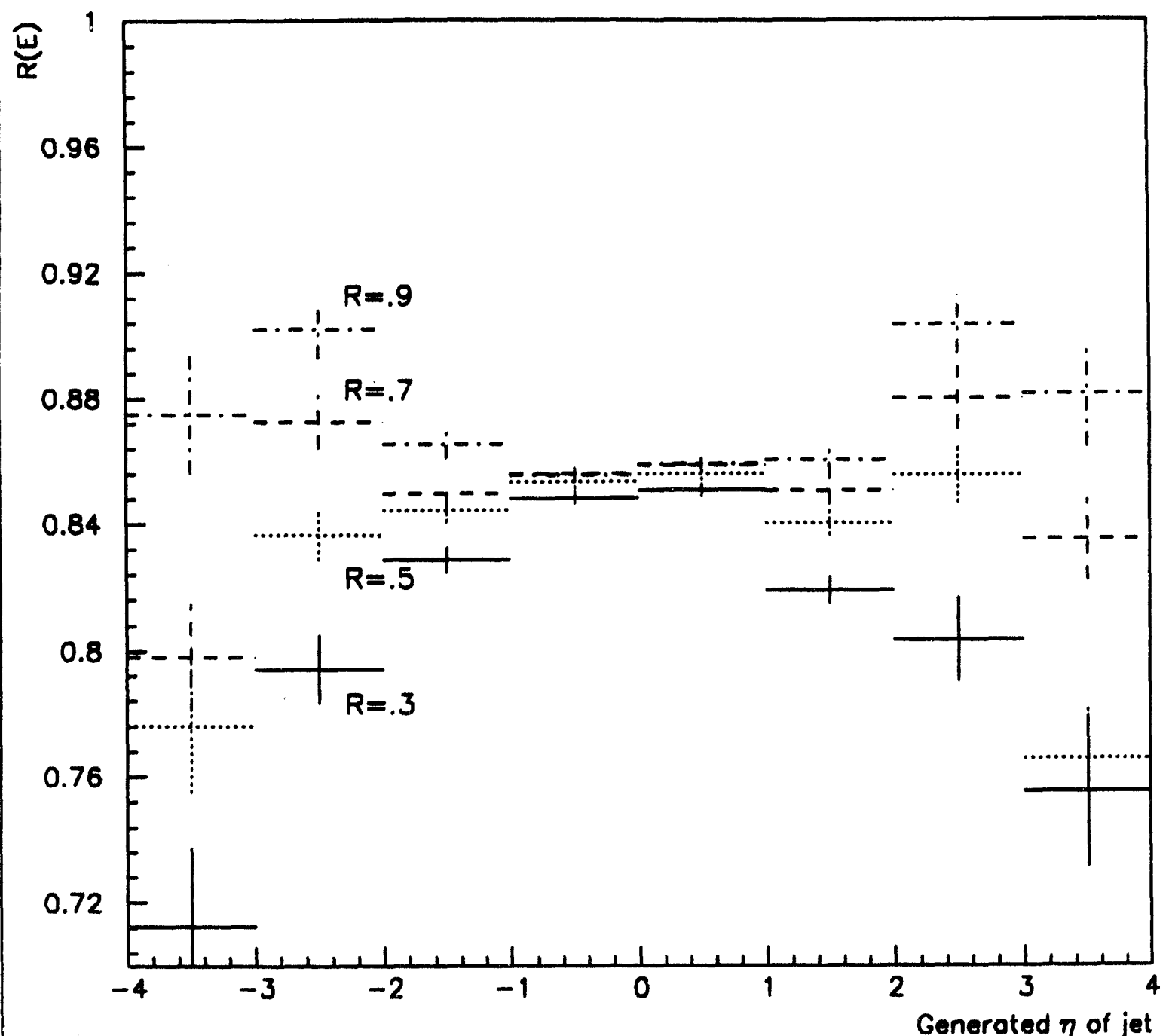

Figure 8-6: Dependence of $R(E)$ with cone size. The dependence is minimal in the central region but large in the forward regions 
Note

There is also a bias away from the beam line for $\eta$ reconstruction in the forward regions as discussed in chapter 7. This may also be due to the finite width of the particle showers. Indeed, this effect has been seen with single pions in the Monte Carlo ${ }^{2}$. We can understand this as follows: if a particle, moving along a line of constant angle from the beam line, showers symmetrically in a circular region around its original direction, more of the area is contained above that angle than below. See figure:8-7.

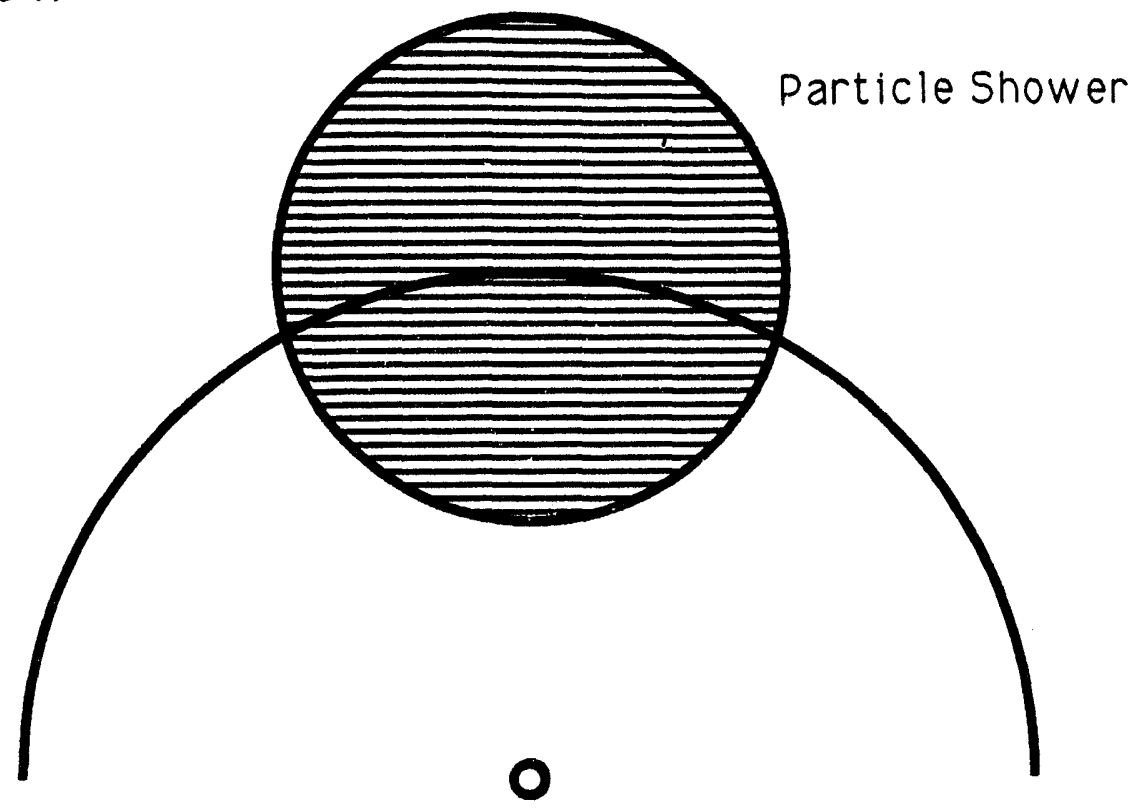

Figure 8-7: A particle showers while moving along a line of constant angle with respect to the beam line. If it does so symmetrically, more energy will be deposited away from the beam line than towards it.

\section{5) Zero suppression.}

Noise in the calorimeter results in energy being seen in nearly all channels even though only a few may actually have had energy deposited by particles. This causes the readout and transfer of large amounts of uninteresting information to the data storage media. To combat this, channels can be forced to zero when they are below some threshold and zero energy channels are not included in the data that is transferred. This is referred to as zero suppression. There was no noise in the calorimeter simulations and no zero suppression used in the jet calibration studies. However, there was a $.10 \mathrm{GeV}$ per readout cell zero suppression that was applied to 
We will confine ourself to methods that can be used at the start of data taking and that make maximum use of test beam results. Thus we assume that we will have access to curves similar to 8-4 and 8-5. We further assume that results that we obtain using the simulation results of 8-4 and 8-5 which are then applied to the simulation, might be equally as good if we took the test beam results and applied them to the real detector.

To verify that energy loss is derived from the causes we expect, we use a technique that we will exploit time and time again. Reconstructed jets are compared with the generated jets which are calculated using the PJET particle method and identical cone sizes. Thus the problem of comparing the generated to reconstructed energy is basically a calibration issue. The jet energy is just the sum of the particles within the jet. We cân obtain this from the generator:

$$
E_{\text {Geaerated }}=\sum_{\text {all particles in jet }} E_{1}
$$

We look at each of the particles that made up the generated jet, and for each one, we can associate a response from the single pion and single electron response curves in figures 8-4 and 8-5. The jet response is then the sum of these terms. We assume that the particles respond according to the curves and add them all up. Thus we end up with a prediction for the reconstructed jet energy. Thus we can predict $R(E)$ for jets:

$$
\left.R(E)_{\text {Predicted }}=\frac{1}{E_{\text {Generated all particles in jet }}} \sum_{i}\left[E_{i}, \eta_{i}, i d_{i}\right)\right]
$$

Where id equals 'electron' if the particle is an electron or a photon and id = 'pion' otherwise. This tells us which curve to use. We can then calibrate the reconstructed jets using this predicted value of $R(E)$. If jet energy loss is truly due completely to the response of single particles, this method should calibrate fairly well. Figures 8-8 describe the percentage energy loss before we calibrate. An average energy loss of about $15-20 \%$ is shown. Figures $8-9$ shows the same quantity after calibration. The calibration removes the energy loss to within $2 \%$ except for $\eta>2$. which suffers from out of cone showering effects which we have not yet tried to correct. Although this method seems to move the response to 1 , it is not useful for real calibration as D0 can not determine the momentum and energy type of every particle that entered the detector. Even if the energy information could be extracted, the id would remain unknown. Some experiments operate under the assumption that jets behave as if they were composed entirely of pions. Figures 8-10 show the results of the calibration 


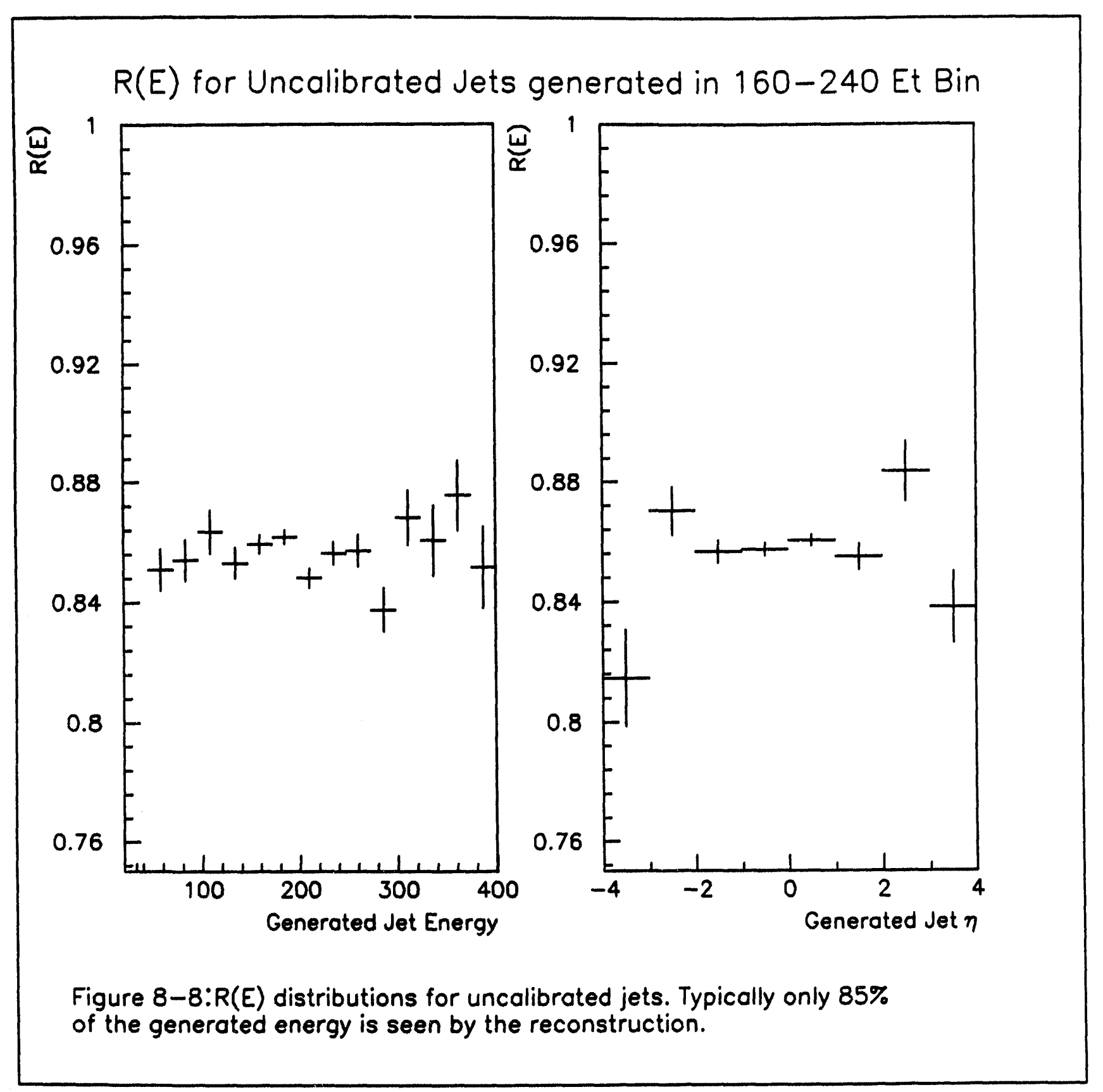


$R(E)$ for Single Particle Calibrated Jets (160-240 Et Bin)
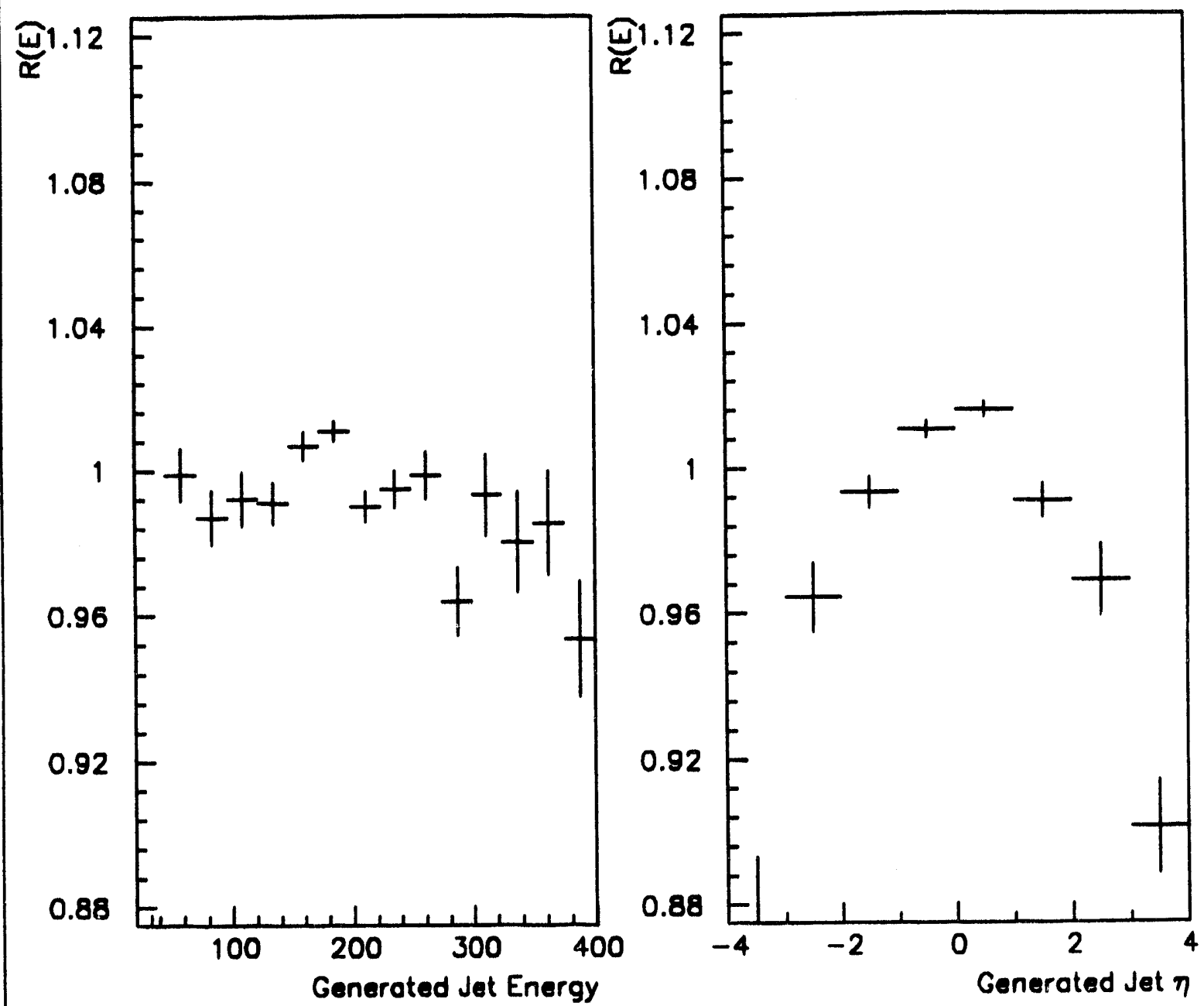

Figure $8-9: R(E)$ after calibration using knowledge of the energy and type of the particles thot make up each jet. 


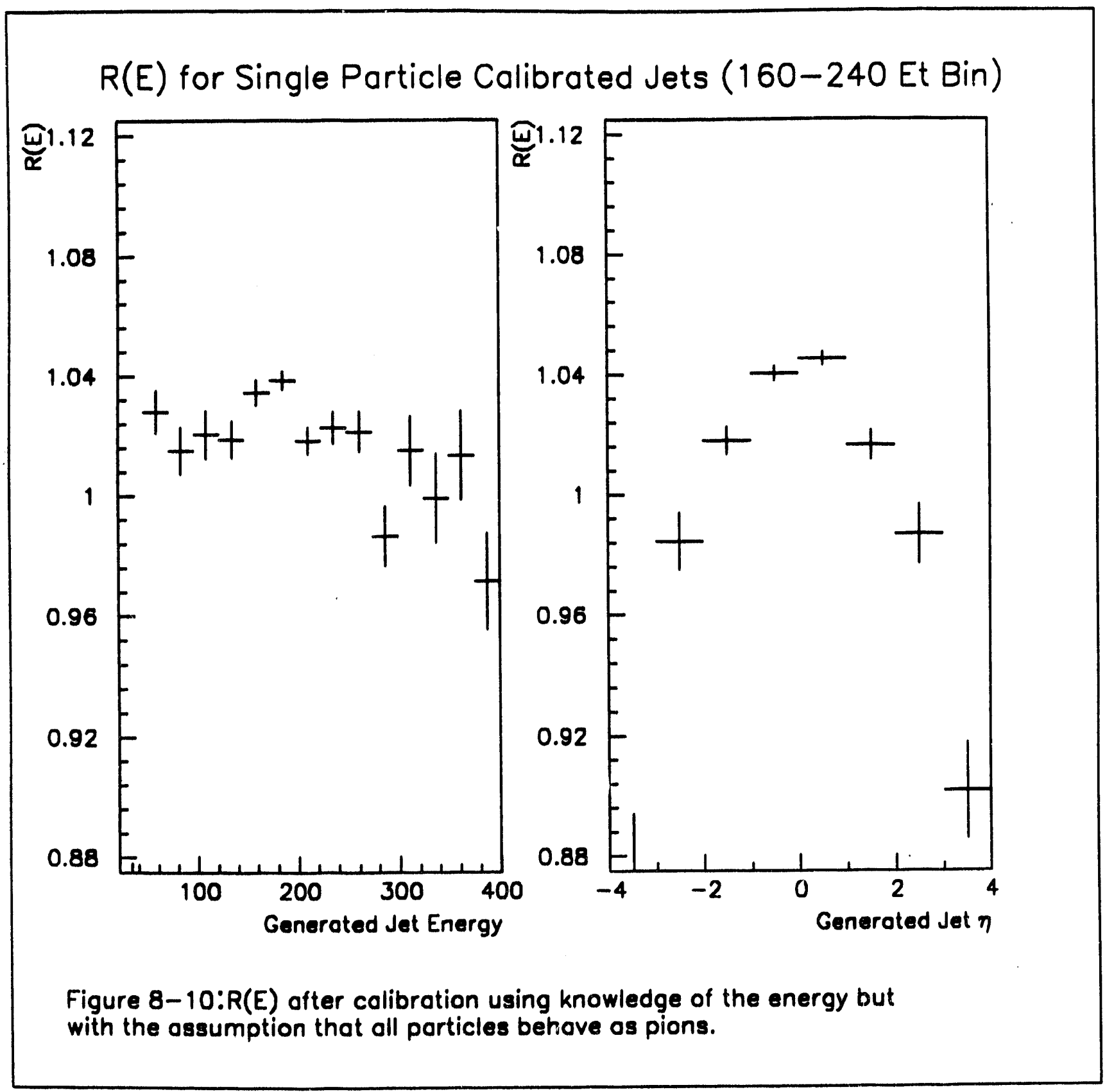




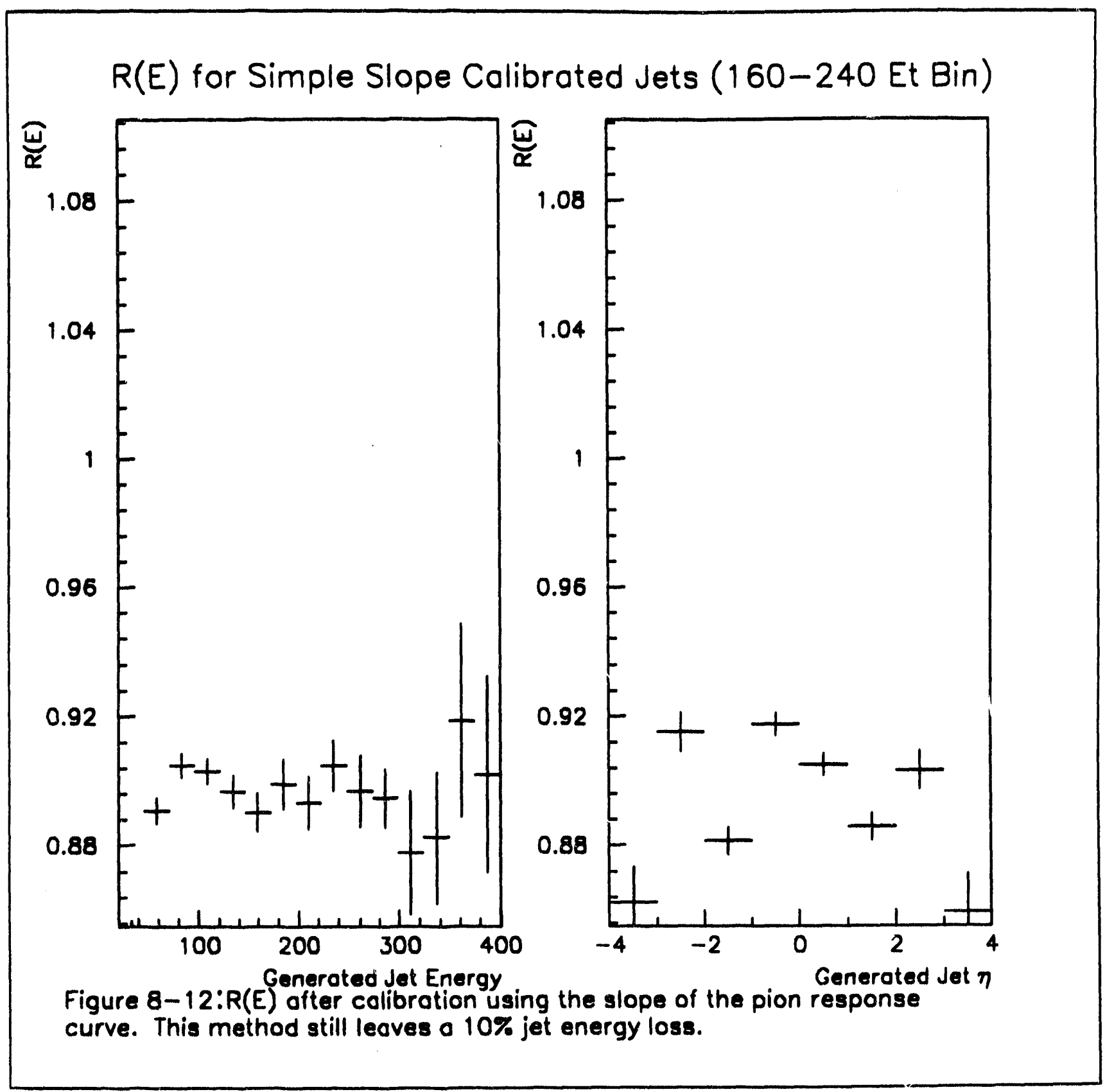




\section{Method 1: Particle Tracking}

One method that was developed entailed using the tracking chambers to identify the energies of particles within the jet. Clusters of energy were matched with central detector tracks. If the energy cluster could not be matched it was shared with adjacent clusters if possible, if it was isolated, it was assumed to be a neutral particle and a 'pseudo' track was created and pointed towards that cluster. See figure 8-11. The solid lines represent charged tracks w'tlle the dashed lines represent 'pseudo tracks'.

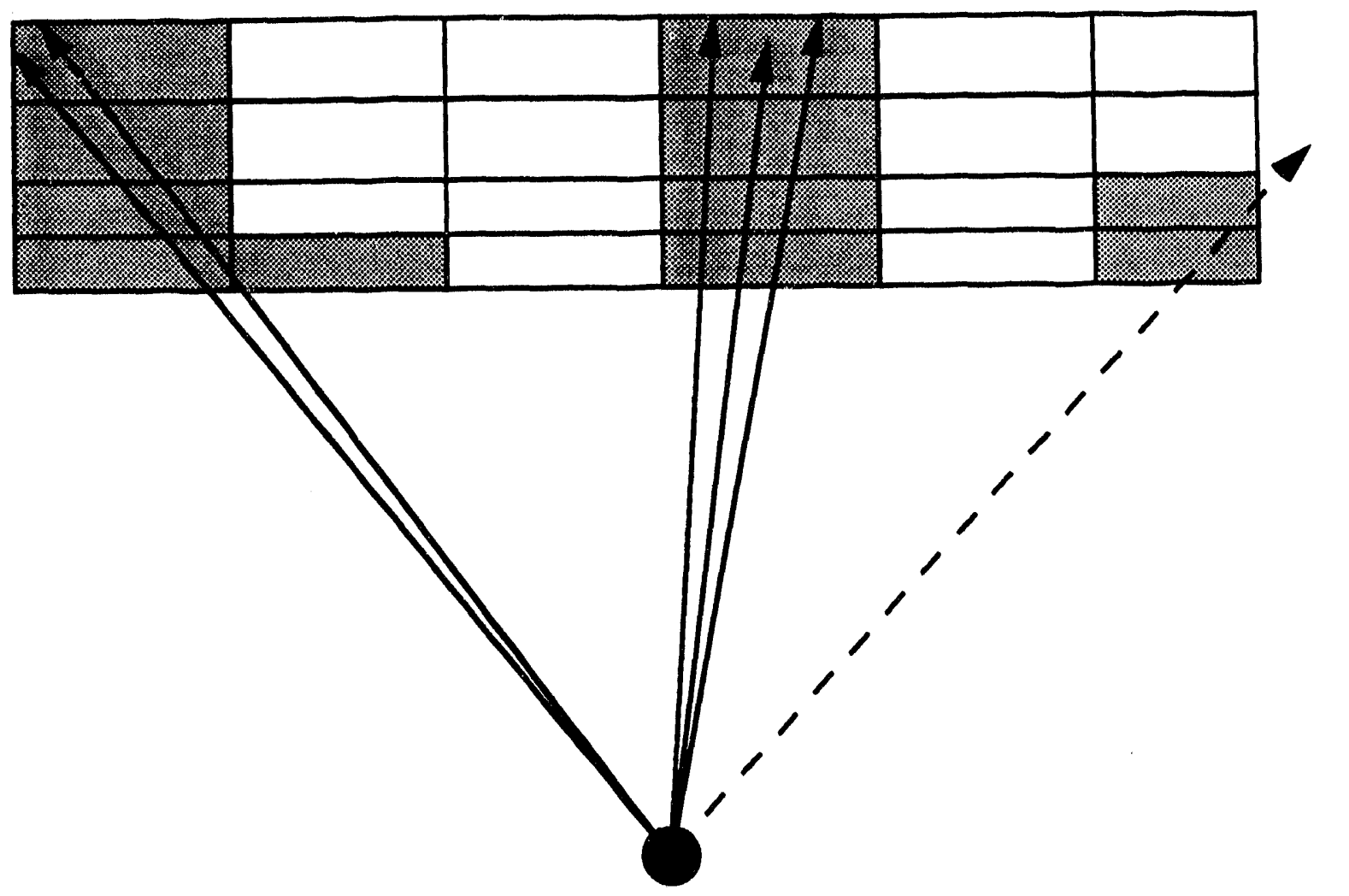

Figure 8-11: Example of how the Particle Tracking Method for Jet Calibration is done. The tracker supplies trajectories of charged tracks that are associated with clusters of energy in the calorimeter. If a cluster has no track match, it is assumed to come from a neutral particle and a 'pseudo' track is created.

The charged tracks were provided by the event generator in this analysis instead of the central detector. A further decision had to be made in the case that more than one track pointed to the same energy cluster. In one case the energy was shared among tracks which indicated particles with equal energies. In the other case the energy was assumed to belong to only one particle. In both cases, it was assumed that $1 / 3$ of the 
jet was electromagnetic by using as the response: two thirds of the pion response added to one third of the electron response for each energy and pseudo-rapidity point used. Figures 8-13 and 8-14 show the results of these calibrations. Central jets are calibrated to within $4 \%$ although the forward jets will still exhibit losses due to out of cone showering. The two differ by only $2 \%$ at most and give us a way to calibrate on an event by event basis. Unfortunately, it requires full track reconstruction and assumes that energy deposits by single particles are reasonably localized. The addition of noise will further complicate matters. It is also not clear whether the method is actually identifying particles within the jet or is simply breaking down the jet in some random way and hence allowing a calibration that moves in the correct direction. This method was not developed further. 


\section{$R(E)$ for Trock Cluster Calibrated Jets (160-240 Et Bin)}

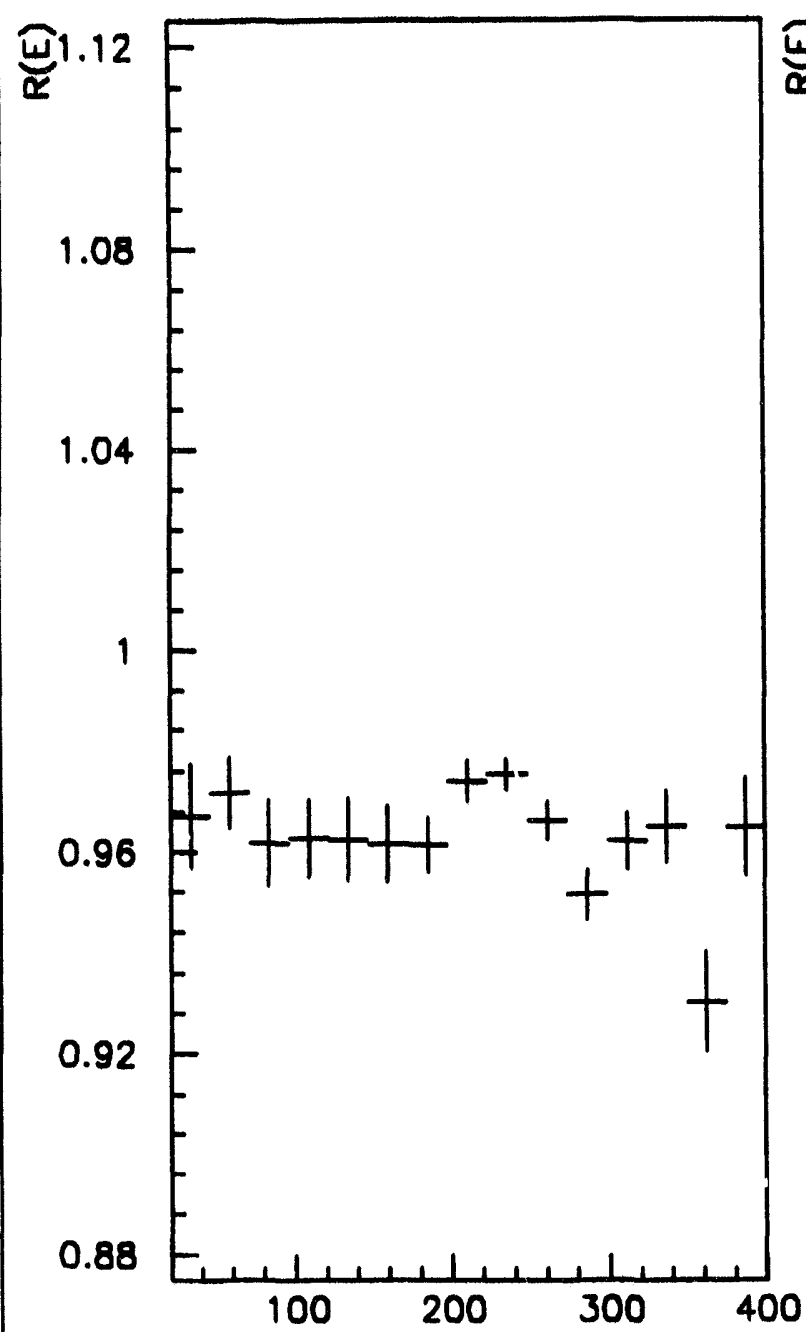

Figure 8-13:R(E) after calibration using the tracking method assuming one porticle contains all the energy of the cluster. 


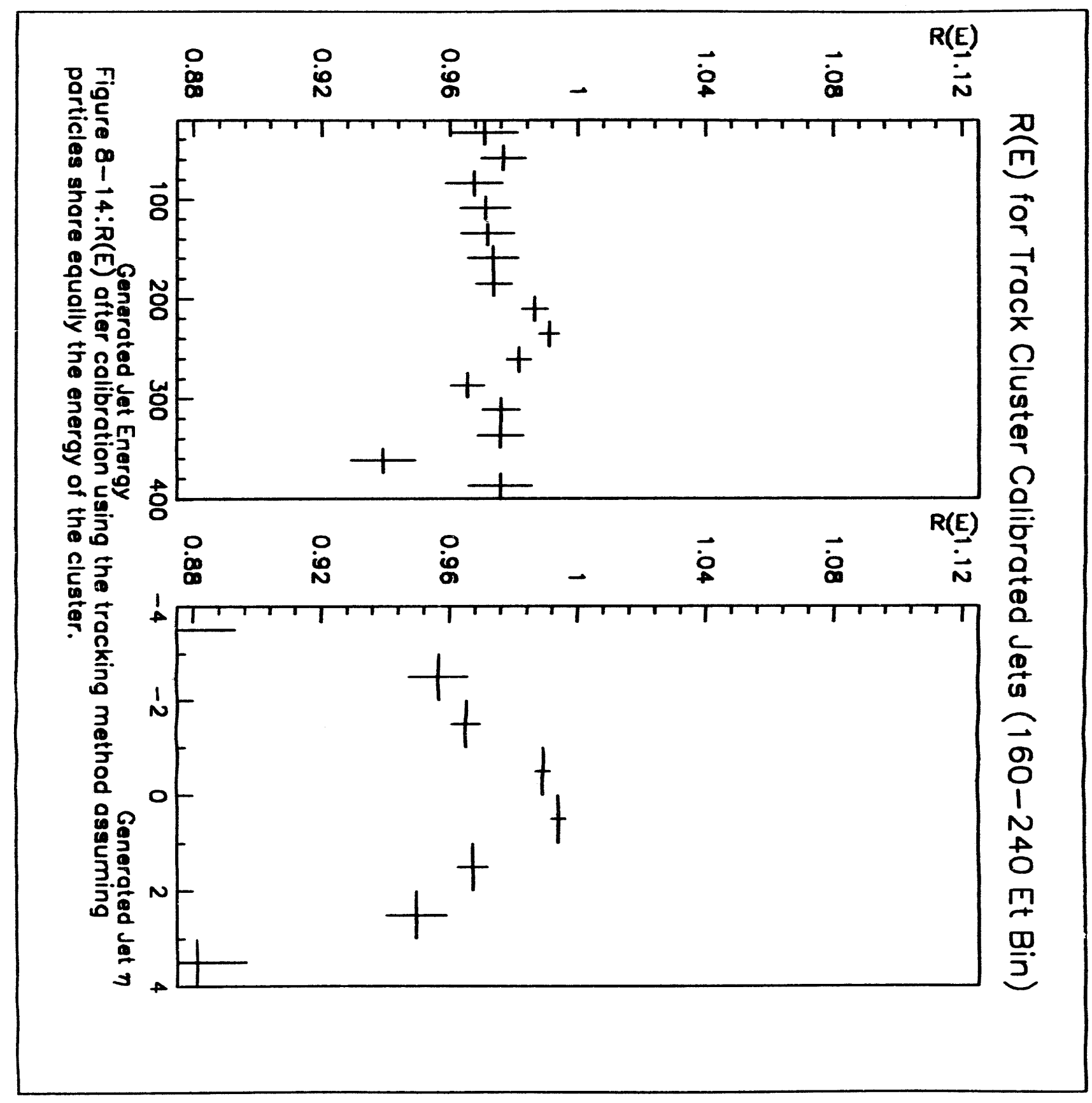


Method 2: Calibration as a function of Jet Characteristic Another method is to base calibration on some jet quantity that is characteristic of the type of particles within the jet. For example, electromagnetic particles respond better than iadronic particles, thus a calibration number could be assigned on the basis of electromagnetic fraction. Also, jets that are very broad tend to be contain softer particles, so jet width could also be a quantity to use for calibration. Figure 8-15 relates the calibration ratio $(R(E))$ versus the electromagnetic fraction and the jet width of both the reconstructed jet and the generated jet.

Let us first consider the electromagnetic fraction plot. It is produced by first looking at each generated-reconstructed jet match. This allows us to determine the $R(E)$ for this pair. We can then determine the electromagnetic fraction of the generated jet by looking at the particles that make up the jet and taking that fraction of the jet's energy that is carried by photons or electrons. This is the electromagnetic fraction of the generated jet. The solid line is for these points and shows that jets that have energetic photons/electrons have a distinctively better energy response (by almost $12 \%$ ) than for totally hadronic jets. The electromagnetic fraction for reconstructed jets is defined similarly but is based on which sections of the calorimeter the jet deposits energy. The dotted curve displays the average value of $R(E)$ for reconstructed jets versus energy fraction. This curve varies only by about 5\%. The difference between the curves comes from the fact that the amount of energy deposited in the electromagnetic sections of the calorimeters is not a good indicator of whether the particles were electromagnetic in nature. Hadrons frequently deposit large amounts of energy in the electromagnetic section just as photons and electrons deposit small fractions in the hadronic layers.

The second plot made in a similar way, but this time versus the width of the generated and reconstructed jet. Jet width in the case of the generated jet is defined by equation 6.1 while the reconstructed jet width is the second moment of ET distribution of the jet in $\eta-\phi$ space. We see that thin jets (small jet widths) respond better than broad jets. Additionally, the reconstructed jet width seems to be comparable with that of the generated in the sense that generated jets of a particular jet width have a similar response to reconstructed jets of the same width. Jet width may be an important quantity to consider in calibrating jets. This method was not developed further however. 


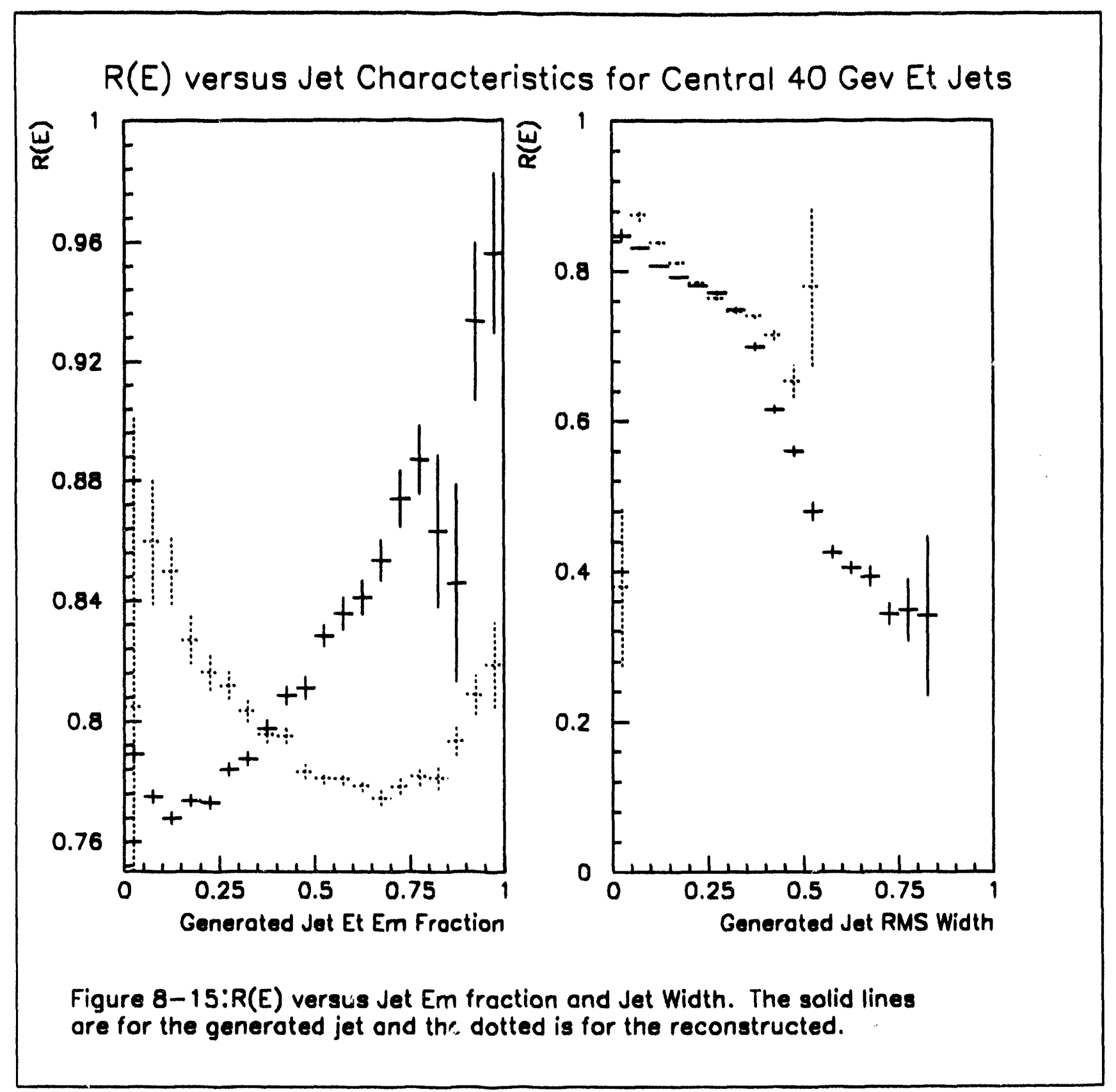




\section{Method 3: Assumed Fragmentation of Jet}

If we assume that we can "understand the behavior of single particles in the detector from the test beam results, we only need to understand how particles are distributed in a jet to be able to predict its response. This was discussed above and seems to be true for jets with $\eta<2$. when a cone of at least $R=.7$ is used. To do this, one must use a generator like ISAJET or HERWIG to model the particle structure of the jet. This may leave the calibration sensitive to which model is used. We can proceed if either we believe that one particular model is reasonably accurate in predicting the particle structure of a jet or if we believe that the predictions are relatively insensitive to which model is used. We can combine the two statements if we test the jet response predictions on several models including one which seems to compare favorably to measurements of jet fragmentation. HERWIG is such a generator. ${ }^{4}$ Figure 8-17 shows the distribution of the predicted response $R(E)$ for various event generators for $50 \mathrm{GeV}$ Et jets generated at $\eta=0$. Note that width of the distribution is only $2 \%$. This is considerably smaller than the width of the $R(E)$ determined from the simulation which is about $10 \%$. Presumably this is because the width is dominated by energy resolution and perhaps out of cone fluctuations.

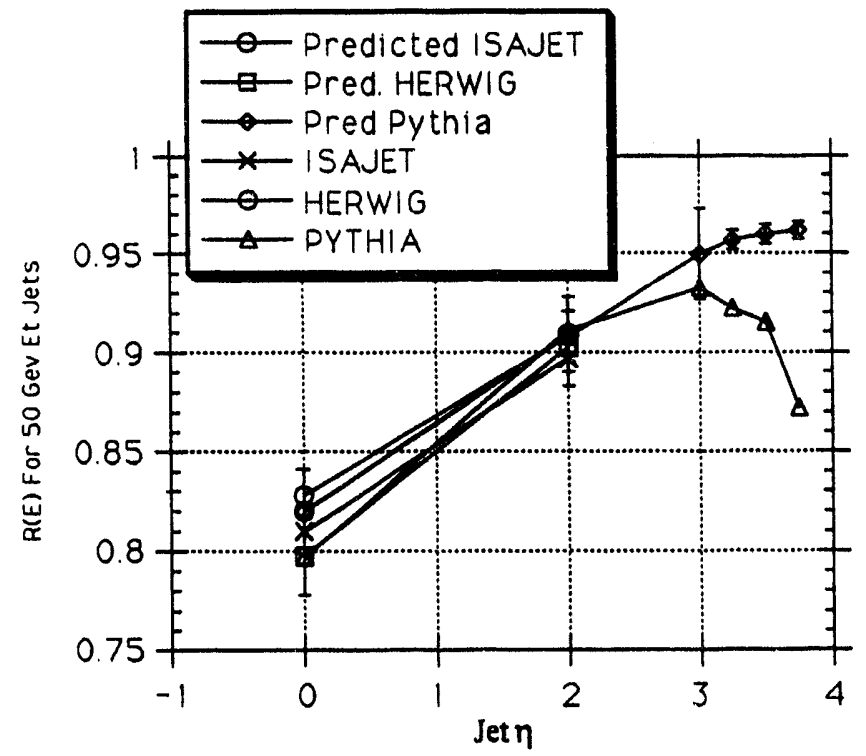

Figure 8-16: $R(E)$ for $50 \mathrm{GeV} E T$ jets at various detector $\eta$. Curves are both predicted and measured for various simulations. Error bars are shown only for predicted values 
Note also, that the different fragmentations agree to within $2 \%$. Figure 8-16 shows this predicted $R(E)$ for jets at fixed $E_{T}$ at various rapidity points. Each data point consists of at least 500 points leading to error bars well within $1 \%$. The simulated values of $R(E)$ have also been plotted. Note that all the points agree within $3 \%$. We conclude that although we do not know which fragmentation model to use, it may be that the fragmentation does not matter to the $3 \%$ level. This is certainly a function of the non-linearity of our detector but given a set of non-linear response curves (as we have assumed here) we can predict this difference. Figure 8-18 shows both predicted $R(E)$ and the Monte Carlo $R(E)$ from a sample of generated jets with $E_{T}$ between 40 and $60 \mathrm{GeV}$. The agreement starts at about $2 \%$ and slowly gets worse until an $\eta$ of 2.5 where the calculated response turns over and drops quickly. Figure 8-19 shows similar results for $\mathrm{R}\left(\mathrm{ET}_{\mathrm{T}}\right)$ done in a similar way. As mentioned above, this is the region where the finite shower width of the jet starts to spread out of the cone. The out of cone issue will be addressed partially later.

Summarizing, this method calibrates jets in the central calorimeter at the $4 \%$ level using only knowledge of the behavior of single particle pions and electrons at 6 different energies and at 5 different pseudorapidity positions in the calorimeter. Since such measurements have been made with real pions and electrons and real modules at the D0 test bean, this calibration method could be very useful as a starting jet calibration upon collider start up and the beginning of data taking at D0 this year. This technique is being currently implemented. 


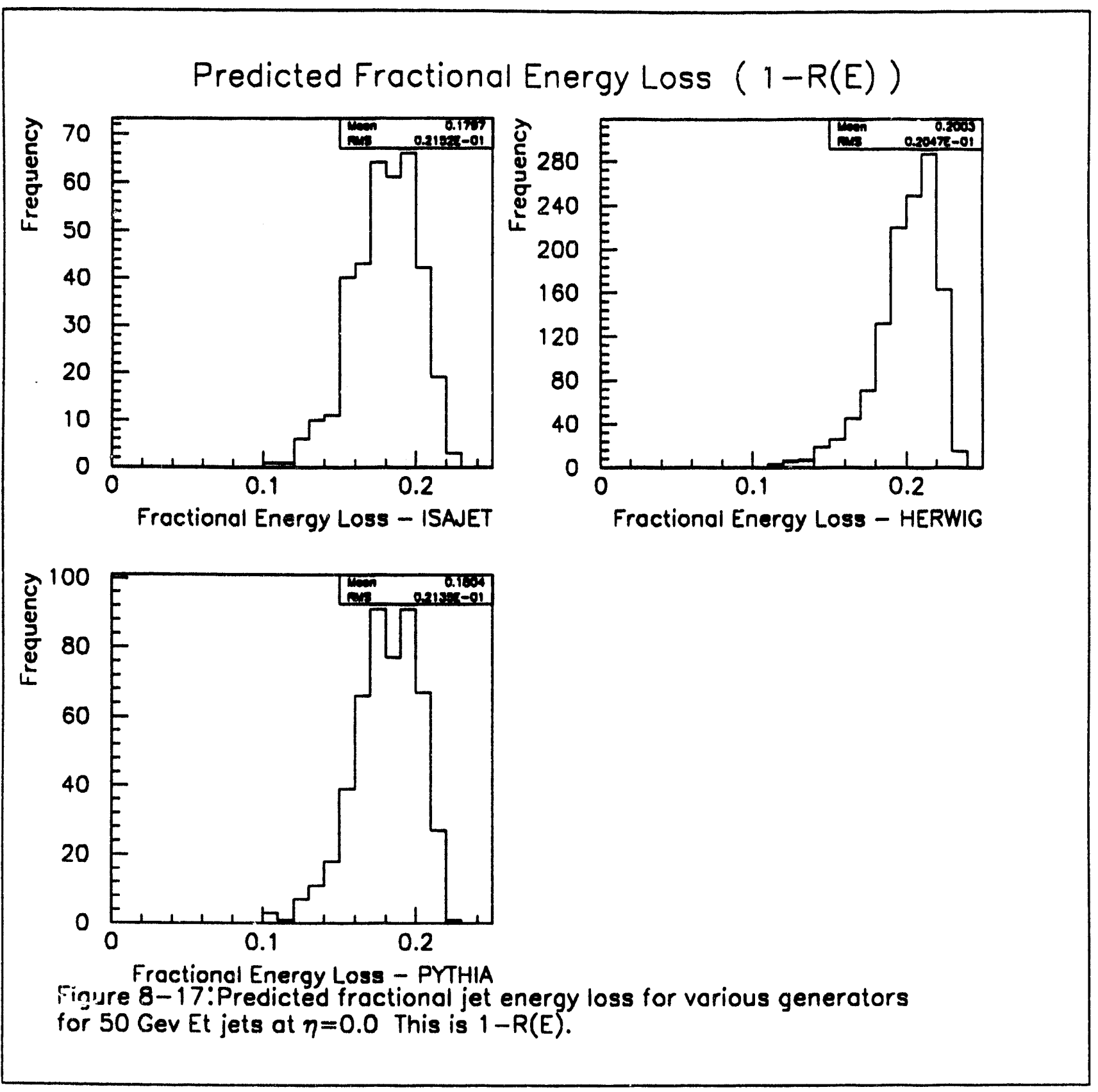




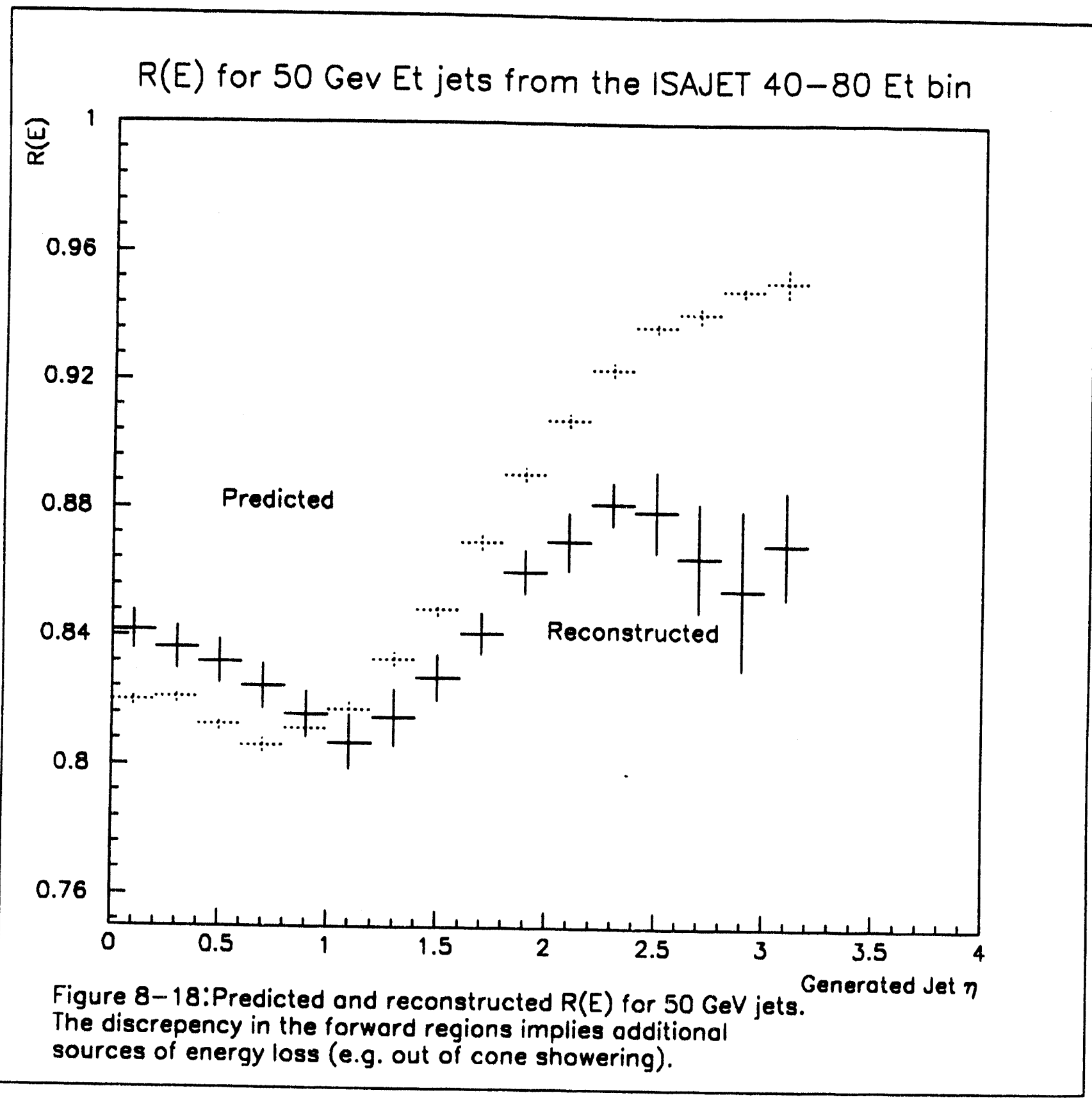




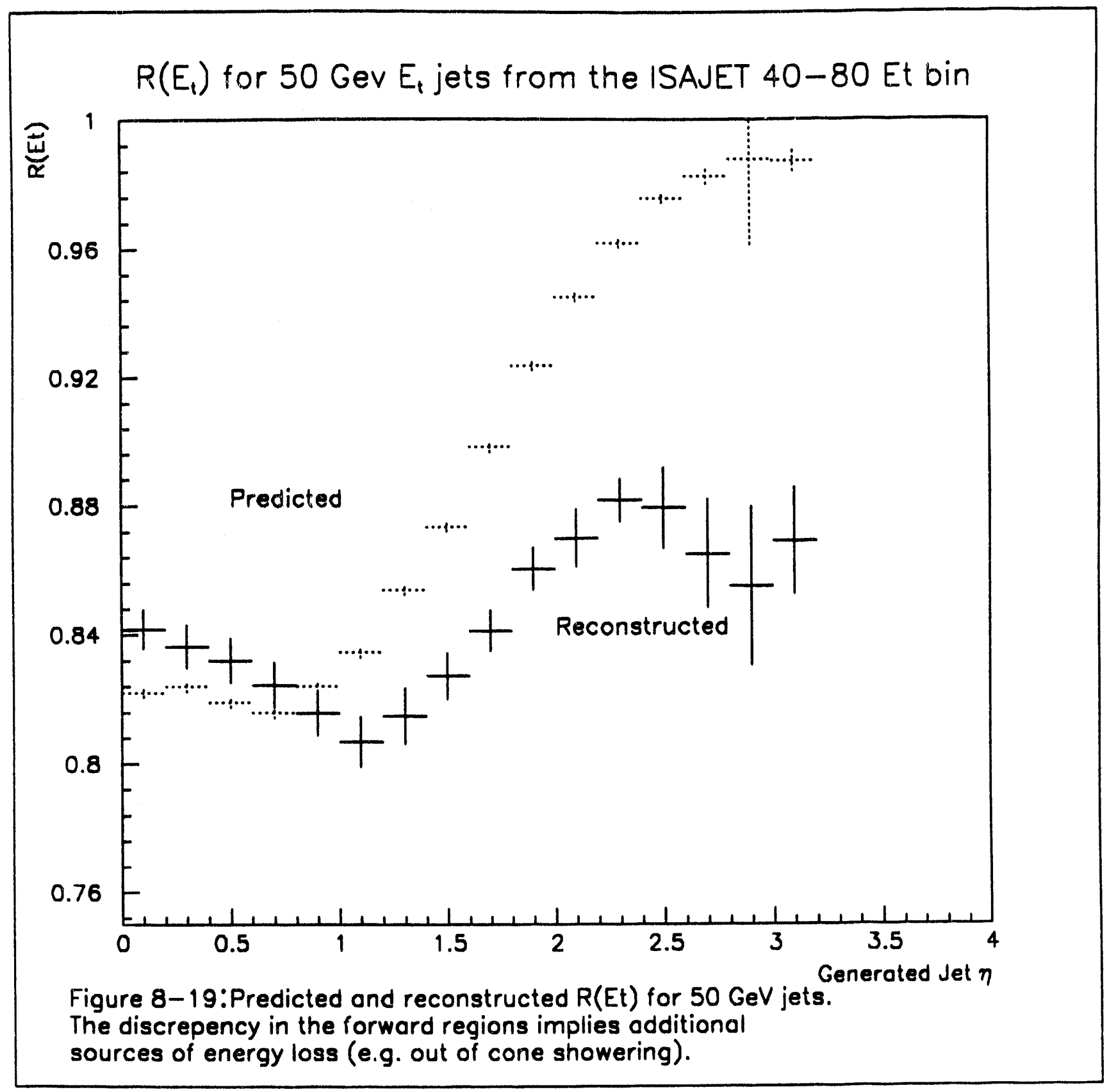


1S.J. Durston, Presented at the DO McPhysics II Conference D0 Note 1202 (1991) Energy Calibration and Systematics

2N. Amos, P. Draper and S. J. Durston, Eta-Dependent Effects Due to Shower Size, D0 Note 1162. D0 Experiment at Fermilab, Batavia, IL. July, 1991.

${ }^{3}$ Current proposal by G. Blazey, G. Forden et al.

${ }^{4}$ CDF Collaboration, Measurement of QCD Jet Broadening in p-pbar Collisions at 1.8 TeV, FERMILAB-PUB-91/23-E (submitted to Phys. Rev. D. 1991) 


\section{Chapter 9: Triggering on Jets at Do}

Protons and antiprotons circulate around the collider in 6 bunches each. At current design specifications, bunches cross at the $\mathrm{D} 0$ interaction point once every $3.5 \times 10^{-6}$ seconds. The cross section for an inelastic (the proton or antiproton breakup) collision is about 50 millibarns. At the expected startup luminosity of $5 \times 10^{30} \mathrm{~cm}^{-2} \mathrm{sec}^{-1}$, this implies a crossing rate of about 250,000 events per second with an average of at least one inelastic collision per crossing and a significant occurance of multiple interactions in a crossing. The rate at which events can be written to tape is expected to be on the order of 1 per second. The job of choosing 1 event out of 250,000 belongs to the D0 Trigger system. We will overview the trigger system and then refocus on their roles in jet selection.

The D0 trigger system primarily consists of 2 levels. The first level is a hardware trigger that consists of an electronic framework which accepts input from any one of four hardware trigger subsystems:

Level 0: The Level 0 trigger consists of two sets of scintillators on either side of the interaction point. This purpose of these scintillators is to detect a charged particles from an interaction, supply the location of the interaction and determine if more than one interaction took place.

TRD: The TRD trigger system can trigger on the presence of charged, relativistic particles (electrons!) that move throughout the transitional radiation detector.

Level 1 calorimeter trigger: This trigger constructs quick sums of calorimeter cells and constructs quantities that can be used for triggering purposes. 
Muon trigger: The Muon Trigger is able to detect events in which hits in the muon system are aligned in such a way that they might point to the nominal interaction point.

There are 256 trigger terms that represent various settings of these 4 trigger subsystems and the Level 1 trigger framework allows the definition of 32 triggers which are made up of any combination of these terms. If any of these 32 trigger conditions are satisfied, than the event is given to the second level (Level 2) of the trigger system for the final decision on whether the event will be accepted or not. The rate into this second level should be reduced to at least $200 \mathrm{hz}$ by this time. Figure 91 is a diagram of the D0 trigger system. Upon a Level 1 trigger, the framework causes the readout and digitizing of a set of detector ADC crates which were associated with that specific trigger. Special processors sequence this data and transfer it along one of 8 data cables. The event is sent to one of 50 microvax 4000 model 60's. Each one is approximately a $16(\mathrm{VuP}) \mathrm{MiP}$ machine. Before processing occurs, the event is built in a format that is identical to that which is used in offline analysis. At this level, the event can be reconstructed using FORTRAN routines 1 which are similar in function to those used offline. A complicated set of parameter sets for each routine decides whether the event is to be written to tape. These routines give answers to yes/no type questions such as "Were there 3 jets found with at least $20 \mathrm{GeV}$ in ET?" or "Was an electron found?". A Level 2 trigger requirement is often referred to as a script and consists of a series of calls to these routines. It requires a 'yes' to all the questions asked in order to 'pass' the script. A total of 128 such scripts may exist. We will review the trigger system in more detail, this time concentrating on jet triggering. 


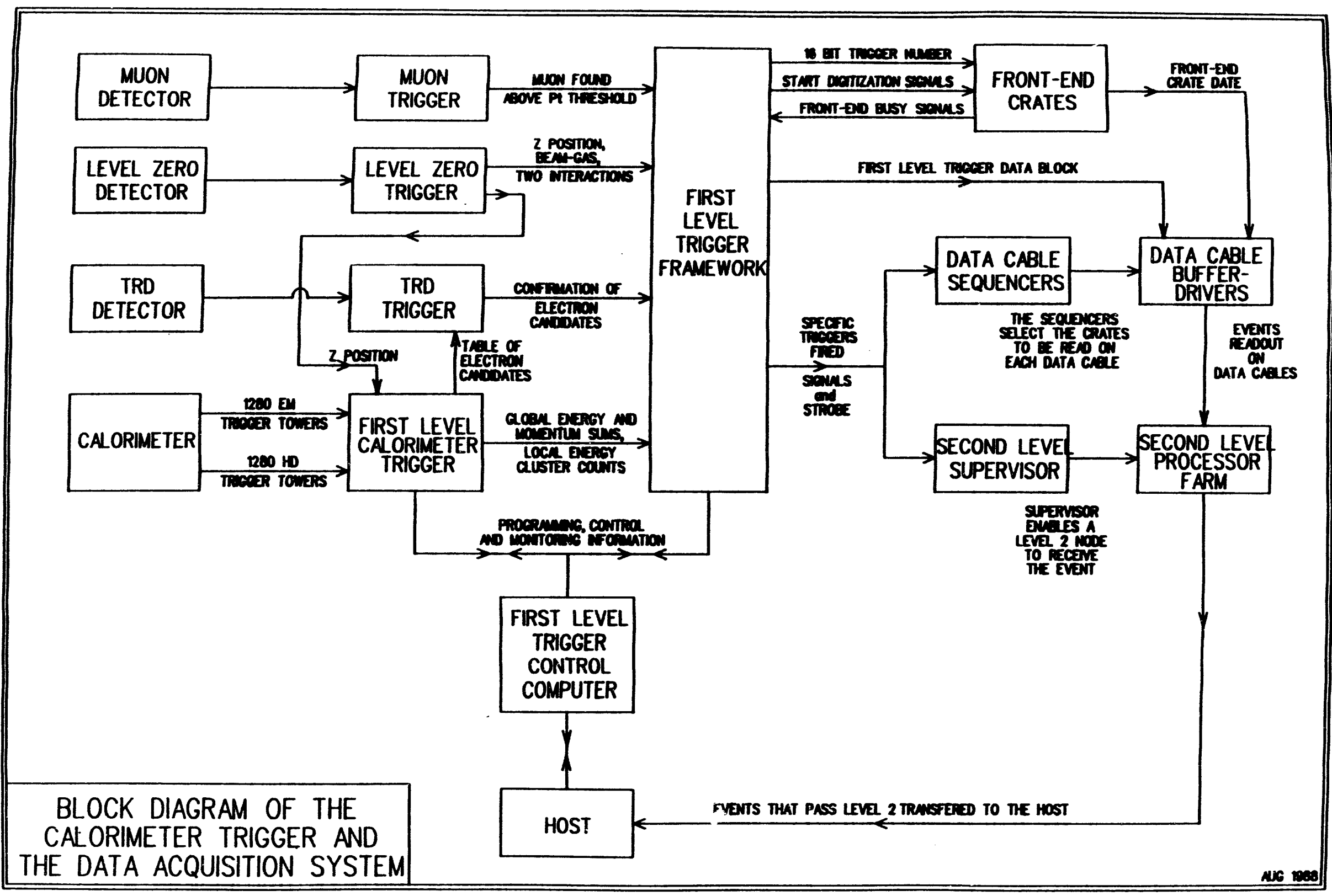

figure 9-1: Du Trigger and Data Acquisition Svstem 


\section{Level $0^{2}$}

The Level 0 scintillators are primarily responsible for flagging crossings in which the two colliding baryons both fragmented although at the expected peak luminosity, this is the rule rather than the exception. Still, the luminosity changes during the run and the probability of inelastic collisions will change as it does. The Level 0 is to be used in coincidence with a beam crossing signal. In some cases only one of the baryons fragments or else it interacts with some of the nuclei in the evacuated beam tube; the Level 0 is designed to discriminate against such events. As described above, this trigger consists of 2 sets of scintillators located $130 \mathrm{~cm}$ away from the nominal interaction point on each side. Each set is composed of different scintillators at different transverse distances from the beam line. Since the scintillators do not reach all the way to the beam line, beam crossings that do not result in the breakup of the oncoming baryon do not trigger. The Level 0 trigger is set up to be sensitive to the breakup of one of the baryons. As charged particles from the interaction move through each scintillator set, the time of the hits are compared and a time difference between scintillator sets is used to determine whether both scintillators were indeed hit and whether the time difference is consistent with an interaction point within some distance of the nominal interaction point. A slower analysis of these hits can mark the presence of additional interactions in the event. It is planned to use these features to obtain 'higher quality' jet events by triggering on events which have only 1 interaction and the vertex is very close to nominal for high rate jet physics events. The Level 0 was not simulated in any of the analysis presented here. The lack of a Level 0 simulation means that we knew our luminosity exactly. Additionally, the simulation did not incorporate multiple interaction events or events where there were soft collisions between the protons.

\section{Level 1 Calorimeter Trigger}

We are particularly interested in events where there was a hard scattering (not just breakup of the proton and antiproton) between colliding partons. We further restrict our attention to those cases in which the scattering partons had significant transverse energy. This is necessary both because our detector only covers pseudorapidity down to 4 . and because scattered partons that moved along the beam line would be hard to distinguish from the beam jets. It is the job of the Level 1 Calorimeter trigger to distinguish those events which contain jets or other objects with large amounts of ET. We will discuss the features that are directly applicable to jets and how they allow us to select jets of a particular $\mathrm{E}_{\mathrm{T}}$ range while rejecting those of a 
smaller ET range. Appendix $\mathrm{C}$ discusses the stability of our results with respect to fragmentation scheme, calorimeter resolution and noise. We expect that although the relative results of our simulations will predict what is seen at the collider, the overall rate scale may differ by as much as an order of magnitude.

Traditionally, hadron colliders have used triggers based on the total ET of the event to select high ET jet events. This capability exists at D0 as well; indeed, D0's extended pseudo-rapidity coverage provides it with a better than average measure of the event $\mathrm{E}_{\mathrm{T}}$. However, the total $\mathrm{E}_{\mathrm{T}}$ is not a good indicator of the $\mathrm{E}_{\mathrm{T}}$ of the jets involved and our high event rate requires the use of a more specific trigger. As the definition of jets is vague, triggering on jets is not trivial. Jets can be narrow like a single particle, or can be broad and cover large portions of the detector. Ideally, one would like to sum up adjoining energetic cells in hardware and hence identify the jet. This is difficult to implement in hardware and DO has adopted a simpler approach. The Level 1 Calorimeter trigger does a fast sum of the electromagnetic and fine hadronic layers in each $\phi$ and $\eta$ readout tower of the calorimeter. The coarse hadronic, massless gaps and inter-cryostat detector are excluded in this sum. Additionally, each $2 \times 2$ group of these sums ( $2 \times .2$ in $\eta-\phi$ space) is combined into a larger 'trigger tower'. There is an electromagnetic trigger tower and a total (electromagnetic + hadronic) tower. Each sum is weighted by a number that corresponds to the sine of the theta location of that tower using a vertex dependent lookup table which groups the vertex into bins of $30 \mathrm{~cm}$. Figure $9-2$ shows the segmentation of $\eta$ space as seen by the Level 1 Calorimeter trigger. As each trigger tower is $.2 x .2$ in size, 40 trigger towers cover the region from $\eta--4$. to $\eta-4$. while 32 trigger towers cover tine entire azimuth range for a total of 1280 trigger towers. 


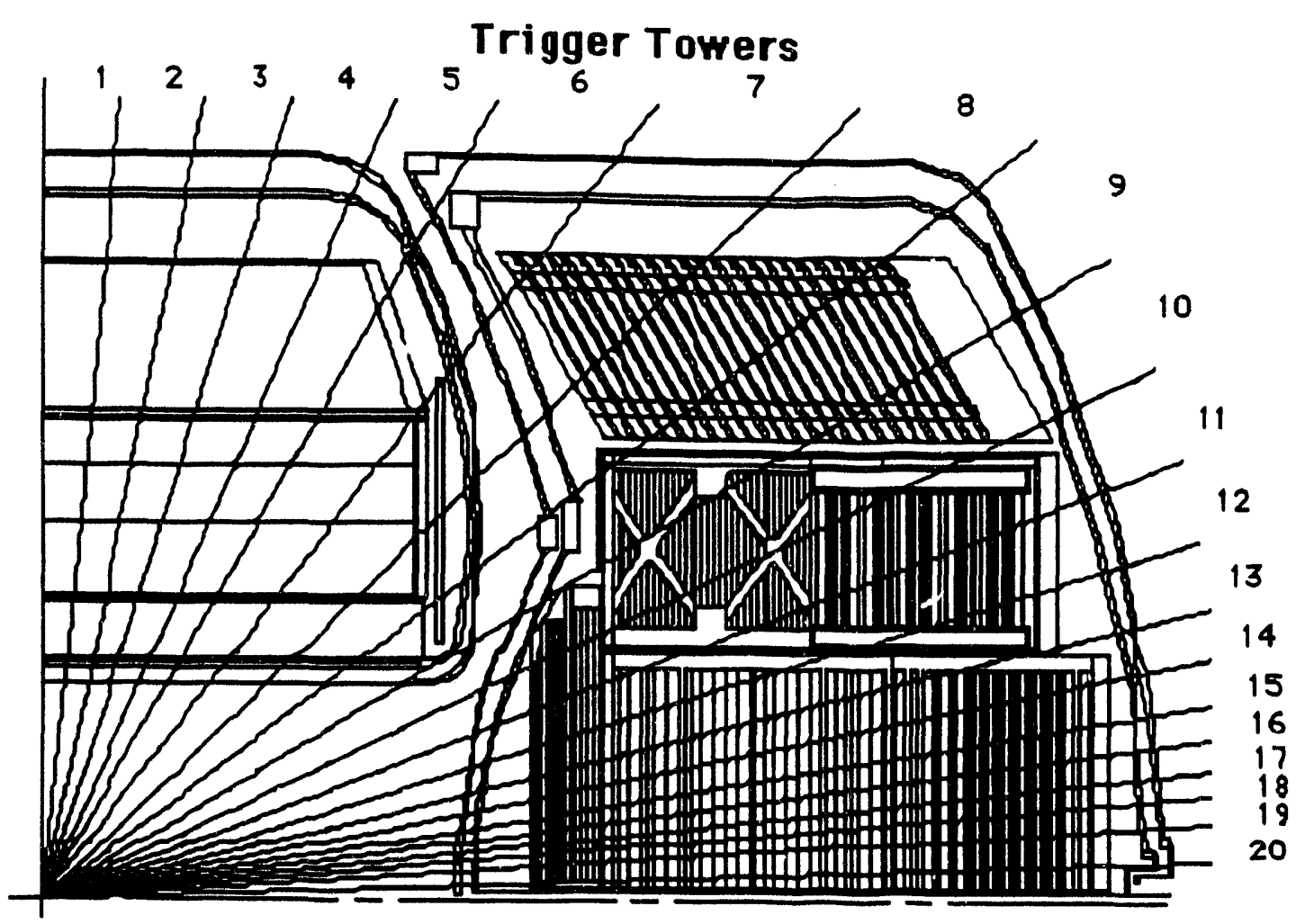

Figure 9-2: The $\eta$ segmentation of the detector at the trigger tower level.

The Level 1 trigger uses these sums to construct such quantities as total ET, total $E_{X}$, total $E_{y}$ and the corresponding vector quantities as well. These quantities can be used to make triggering decisions. However, the most important trigger for jets is the ability of the Level 1 calorimeter to make a comparison of each of the 1280 trigger towers to a threshold. The system can be programmed to recognize when some number of these trigger towers is above that threshold. We refer to this threshold as the Level 1 jet ET threshold and the number requirement as the Level 1 jet count. Note that the word 'jet' here is misleading as the Level 1 has no ability at all to identify a jet, but can only identify trigger towers whose total ET is above the Level 1 jet ET threshold.

But jets seldom deposit all their energy into one trigger tower. Computer simulations have shown that jets deposit on average $1 / 3$ of their energy into the most energetic trigger tower ${ }^{3}$. The distribution is not a delta function however; some jets can deposit all their energy into one trigger tower while others can deposit less than $10 \%$ in the most energetic tower.. Figure 9-3 shows the ratio of the energy contained in the jets most energetic trigger tower to the total energy of the jet itself. This distribution is broad. Nevertheless, it is apparent that a trigger that asks for $10 \mathrm{GeV}$ in 
one tower will not trigger on anything less than $10 \mathrm{GeV}$ jets. However, it is not true that it will trigger on all $10 \mathrm{GeV}$ jets. The requirement that a certain amount of the jet's energy be localized in one trigger tower is a severe one. ${ }^{4}$ Figure $9-4$ shows that simply requiring $7 \mathrm{GeV}$ in a trigger tower precludes full trigger efficiency until 40 $\mathrm{GeV}$ or so. Another approach that can be tried is to require more than one tower above threshold. Instead of requiring 1 tower with more than $10 \mathrm{GeV}$, one could require 4 towers with $2.5 \mathrm{GeV}$ each. In each case $10 \mathrm{GeV}$ is asked for, but the latter case is much more efficient for $10 \mathrm{GeV}$ jets. But since the Level 1 has no idea whether those trigger towers are adjacent or not, the latter requirement tends to accept a higher rate of lower ET events. We will see that the background rate from lower ET jet events can be very high.

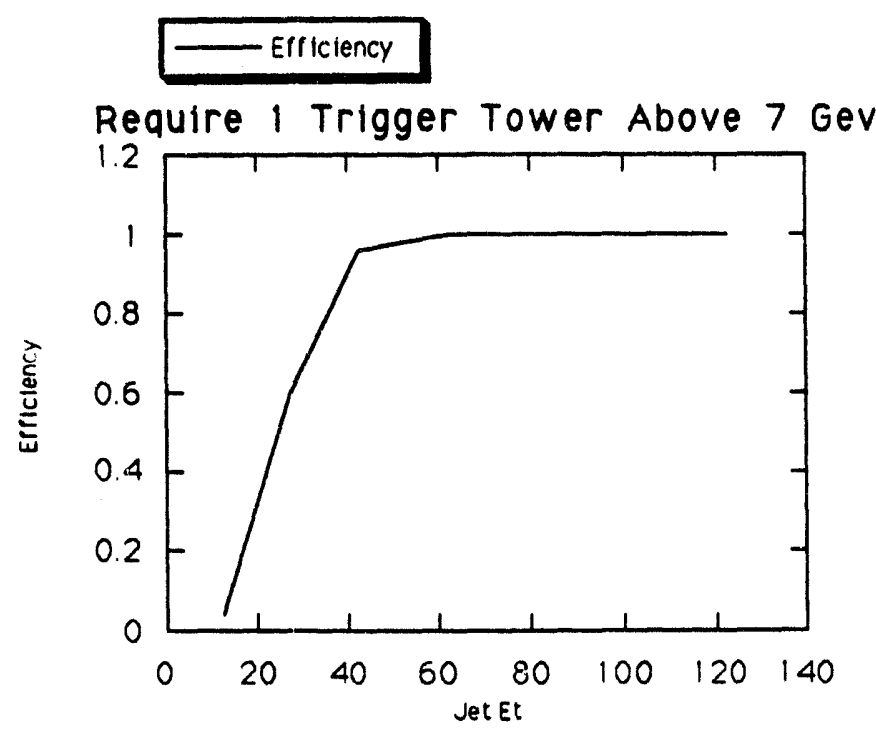

Figure 9-4: Trigger Efficiency versus Jet ET for one particular Level 1 trigger setting. At least one trigger tower whose total $\mathrm{ET}_{\mathrm{T}}$ is above $7 \mathrm{GeV}$ is required. 


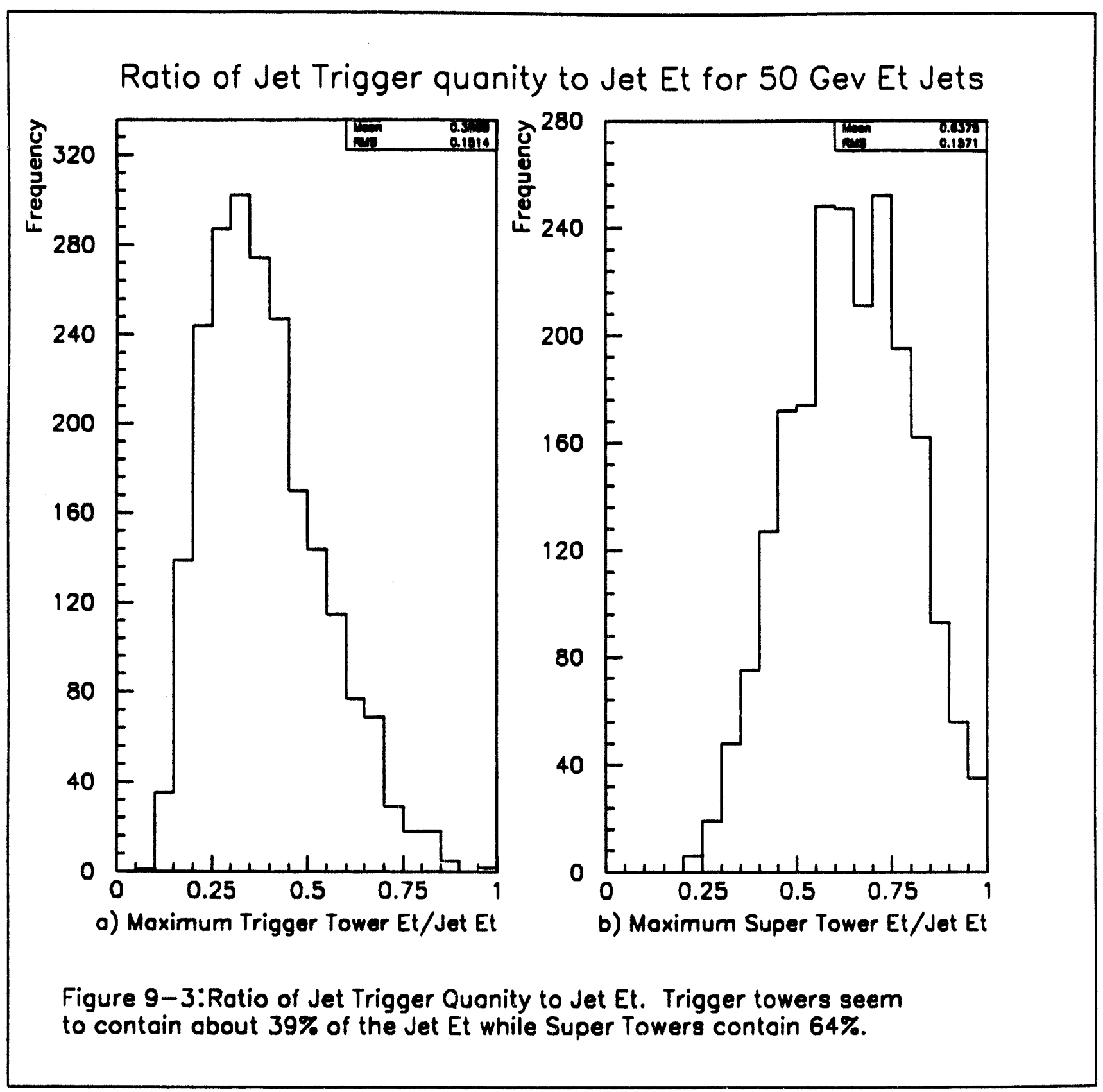


As the jet $E_{T}$ spectrum falls as $E_{T}$ to the - 6 th power, a trigger which was efficient for $30 \mathrm{GeV}$ jets would rarely see an event which had a $80 \mathrm{GeV}$ jet present. We plan to obtain samples of different portions of the spectrum by defining triggers that target separate jet $E_{T}$ ranges and prescaling them so that we receive approximately the same number of events from each. Figure 9-5 shows the triggering efficiency as a function of jet $\mathrm{E}_{\mathrm{T}}$ for various trigger settings: 1 trigger tower $>3 \mathrm{GeV}$, 1 trigger tower $>7 \mathrm{GeV}$ and 2 towers $>7 \mathrm{GeV}$. We can see that they become fully efficient for 25,45 and $80 \mathrm{GeV}$ respectively. We require full efficiency to eliminate a bias in the types of jets we receive in our events.

Thus we are left with the guideline that to select jets with a particular ET, we need to set the trigger threshold at some small fraction of the jet's ET to be efficient. Unfortunately, this allows the trigger to be flooded with events whose leading jet is less than the target $E_{T}$. Consider as an example: The QCD jet ET spectrum falls almost as $\mathrm{ET}_{\mathrm{T}}{ }^{6}$. We wish to trigger on $40 \mathrm{GeV}$ jets and decide that since a $40 \mathrm{GeV}$ jet averages $1 / 3$ of its energy in one trigger tower, we will ask for 1 trigger tower with ET greater than $10 \mathrm{GeV}$ as our trigger. Thus we might get $90 \%$ of that signal according to figure $9-3$. But a $20 \mathrm{GeV}$ jet will deposit $10 \mathrm{GeV}$ about $20 \%$ of the time according to the same plot. Since the cross section is falling like $\mathrm{E}_{\mathrm{T}}{ }^{6}$ we expect the signal to noise ratio for $20 \mathrm{GeV}$ jets alone to be :

$$
\begin{aligned}
\text { signal } & =90 \% \times \sigma(\mathrm{ET}=40) \\
\text { noise } & =20 \% \times \sigma(\mathrm{ET}=20) \\
\rightarrow \text { signal } / \text { noise }= & 4.5 /(26)\left(\text { assuming sigma }-\mathrm{ET}^{-6}\right) \\
& =4.5 / 64=.07
\end{aligned}
$$




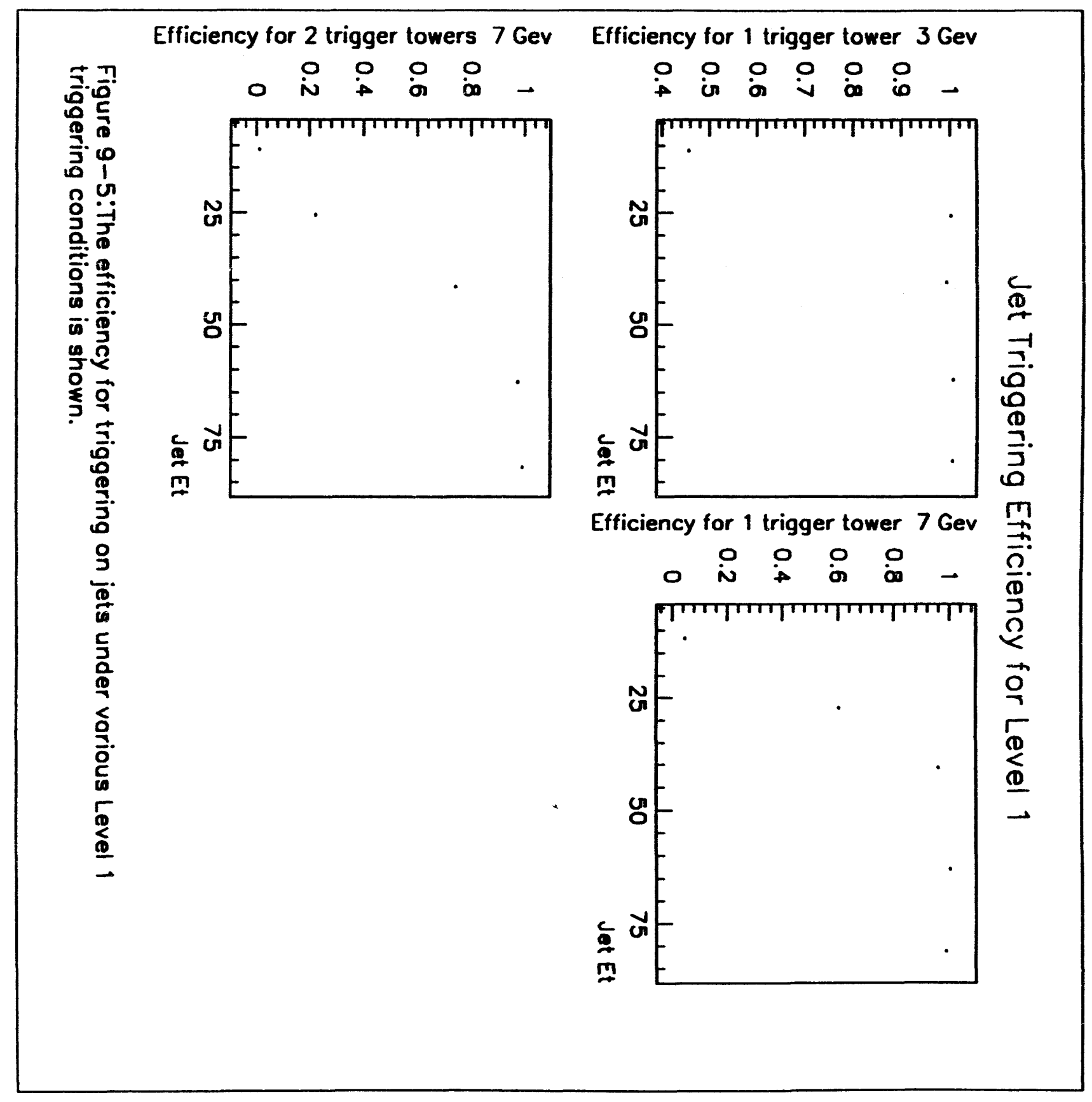


Thus most of the background will come from lower ET jets that are narrower than the ones we are triggering upon. This is the leading cause of 'jet noise' in the trigger. But the amount of background is anticipated to be larger than implied above. This is because we are forced to lower the trigger threshold even further due to trigger and calorimeter imperfections. First of all, the trigger only sees energy deposited in the electromagnetic and the fine hadronic sections. The coarse hadronic in all three calorimeters is left out. as is the massless gap and inter-cryostat detector. This means that jets which deposit large amounts of energy in these regions will be discriminated against. To correct for this, the threshold must be lowered. Further, the vertex correction used by Level 1 is coarse and thus this leads to some ET smearing. Even if we could measure a jet energies exactly, smearing in the calculated ET would result in pulling some jets below our cuts. On top of this, there is finite calorimeter resolution already. The level 1 calorimeter trigger simulation included additional noise which is expected to be present due to electronics and the decay of ine uranium. All of these factors drive the selection of the trigger tower threshold downward. At the same time, the resulting event rate increases dramatically as shown in figure 9-6.

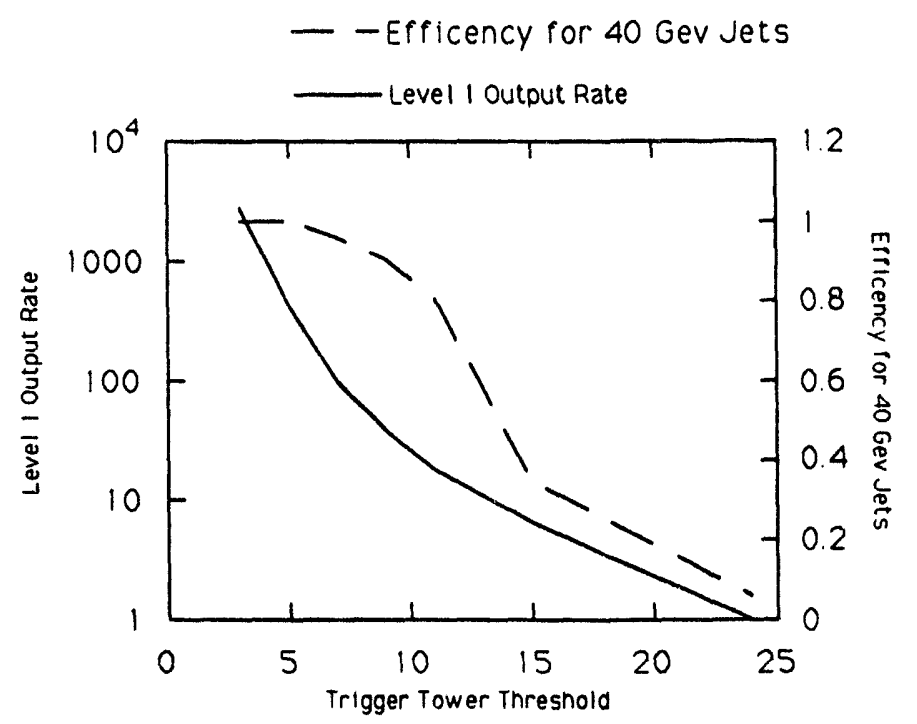

Figure 9-6: As the trigger tower threshold increases the event rate falls but the efficiency drops.

We have seen that in order to be efficient for events with a desired jet ET, we must lower the trigger tower threshold with the resulting rate contamination by lower ET jets. Table 9-1 shows a proposed set of $\mathrm{Ll}$ trigger settings, the jet transverse 
energies they are designed to select and the resulting signal to noise ratios. These trigger settings are fully efficient for jets at or above the target ET. Note the low signal to noise ratio from the large fraction of lower ET jet events which are able to satisfy these triggers due to the rapidly falling jet ET spectrum.

\begin{tabular}{|l|l|l|l|l|}
\hline Target Jet ET & L1 Trigger & Signal Rate & Total Rate & signal/noise \\
\hline 25 & 1 tower $>3$ & 120 & 15900 & .008 \\
40 & 1 tower $>7$ & 14.8 & 600 & .025 \\
90 & 2 towers $>7$ & .36 & 70 & .005 \\
110 & 3 towers $>7$ & .027 & 13 & .002 \\
155 & 4 towers $>7$ & .003 & 3.4 & .0009 \\
\hline
\end{tabular}

Table 9-1: Proposed L1 triggers and rates. Note that the signal rate is a small fraction of the total rate allowed by the trigger. The triggers are fully efficient for jets above the target ET.

We would like to complete our discussion of jet triggering with Level 1 by discussing two miscellaneous topics. The first has to do with jet efficiencies and the second has to do with the possibility of triggering on very low ET jets.

Although we have found trigger configurations that are fully efficient on the average, there might be certain types of jets for which we are very inefficient. One example would be jets that are pointed towards the region where the massless gap and Inter-Cryostat Detector are located; neither is included in the trigger ET sums. If this happens $1 \%$ of the time, a complete inefficiency there might show up as $99 \%$ efficiency overall. However, studies have shown that the triggers tend to be very flat as a function of rapidity. This comes from our definition of trigger efficiency. We define our trigger efficiency for a target jet as the number of events in which the target jet was present and the trigger was satisfied divided by the number of events in which the target jet was present. Note that we do not require that the target jet cause the trigger directly. Thus we have been talking about event trigger efficiencies rather than jet trigger efficiencies. Since other jets are always present in the event, the event can still trigger even though the target jet was not involved. Table 9-2 shows both types of efficiencies for various target jet ET and various level 1 trigger settings. 


\begin{tabular}{|l|l|l|l|l|l|l|l|}
\hline$\#$ & $\begin{array}{l}\text { Tower } \\
\text { cut }\end{array}$ & $2 \_5$ & $5 \_10$ & $10 \_15$ & $20 \_25$ & $40 \_45$ & $80 \_85$ \\
\hline 1 & $3 \mathrm{GeV}$ & .19 & .34 & .70 & .98 & 1.0 & 1.0 \\
& & .006 & .05 & .32 & .78 & .97 & .97 \\
\hline 1 & 5 & .03 & .02 & 0.2 & .76 & .99 & 1.0 \\
& & 0. & .006 & 0.11 & .64 & .96 & .97 \\
\hline 1 & 7 & & .002 & .06 & .48 & .97 & 1. \\
& & & .0004 & .03 & .42 & .94 & .97 \\
\hline 1 & 9 & & & .02 & .28 & .92 & 1. \\
& & & & .01 & .25 & .89 & .97 \\
\hline 1 & 11 & & & .004 & .14 & .79 & .99 \\
& & & & .0005 & .07 & .55 & .92 \\
\hline
\end{tabular}

Table 9-2: Event versus trigger efficiencies for various jet ET bins. We show both the efficiency for a trigger in an event which contains a jet of that ET bin (first row) and the efficiency for a jet of that ET bin to directly cause the trigger.

\section{Using Super Towers in Level $1^{5}$}

The Fermilab Tevatron will allow us to see jets as energetic as $400 \mathrm{GeV}$ in ET. We want to obtain a distribution on as wide a range as possible. Although triggering on high ET jets is not normally difficult, triggering on low ET jets can be. Noise and electronic behavior may forbid the use of trigger thresholds below or even at the $3 \mathrm{GeV}$ threshold we have suggested for $25 \mathrm{GeV}$ jets. A modification to the trigger may solve this problem: Since Level 1 calculates the total ET of the event, it sums up the detector in large pieces. The results of these pieces are stored and might be available for use in the trigger for the collider run. At one level of the summation, the pieces cover an area equal to 1.6 by .8 in $\eta-\phi$ space. Since most cone sizes are less than .8, such 'super towers' are guaranteed to contain more than $25-50 \%$ of any jet. Thus instead of asking for $3 \mathrm{GeV}$ in a trigger tower to get $25 \mathrm{GeV}$ jets, one might ask for $12.5 \mathrm{GeV}$ in one of these super towers. The result is a similar efficiency for the jets of interest with a reduction of rate. Figure 9-3 shows the Super Tower ET distribution for $40 \mathrm{GeV}$ jets. While normal trigger towers contain only $38 \%$ of the jet energy, Super Towers contain almost $64 \%$. Studies have shown that for $10 \mathrm{GeV}$ jets, super towers would result in twice the signal to noise as the single tower threshold at $3 \mathrm{GeV}$. However, the $3 \mathrm{GeV}$ threshold should be more sensitive to noise and 
unforeseen problems in the system, so the real benefit of super towers would be realized in the case that low single tower thresholds like $3 \mathrm{GeV}$ cannot be used.

\section{Jet finding in Level 2 : L2JETS 6}

Up to this point in the trigger, there has been no facility to identify the entire $\mathrm{E}_{\mathrm{T}}$ of the jet. In Level 2, however, software routines can be called which can reconstruct the entire event. In particular, a routine named L2JETS is used to reconstruct jets in the calorimeter. Offline routines exist which do an excellent job of finding jets in the calorimeter; but they cannot be used in the Level 2 system as they require large amounts of time and memory. We will give a brief description of L2JETS, some results concerning its performance as both a trigger and a jet finder and finally the reduction in rate we obtain by using L2JETS in addition to Level 1 jet triggers already discussed.

The basic features of L2JETS is as follows:

1) L2JETS uses locations of the trigger towers above threshold from Level 1 as jet candidates. This means that jet efficiency is determined completely at the level 1 level since L2JETS cannot find jets that did not cause triggers.

2) L2JETS applies a cone algorithm to form jets as is done with reconstructed jets in the offline. The size of the cone is a parameter that is trigger

dependent. The jets are reconstructed with the following quantities calculated: the $\eta-\phi$ of the jet axis, the total ET, the electromagnetic fraction and its width. All of these quantities are thus available for triggering purposes. Only one iteration is done to calculate these quantities. This is different than the offline reconstruction which reapplies the cone algorithm until the calculated jet center becomes stable.

3) The trigger tower ET's calculated by Level 1 are available in a special data bank. Thus L2JETS can use these ET values to do its clustering. The advantage of this is that it saves large amounts of unpacking time. The disadvantages is that coarse hadronic and inter-cryostat information is left out. Alternately, L2JETS can use the digitized calorimeter information. The former mode is referred to as NO_CL2 while the latter is named CL2 mode (named after the unpacking package that it uses.) The CL2 package is similar to the offline unpacking, but it ignores the massless gap and inter-cryostat detectors and is much faster.

4) No jet merging or splitting is done in L2JETS. 
L2JETS is intended to provide accurate jet reconstruction while minimizing processing time and memory. L2JETS stores results based on prior calls so that a jet is only reconstructed once unless the reconstruction is intended to be different (different cone sizes for example). The program also stores its PASS/FAIL results and will use them instead of recalculating if needed. Reconstruction results as well as a list of parameter cuts used are stored for comparison with offline, debugging and for use by analysis packages. The time required to reconstruct a jet is a reasonable fraction of the total Level 2 time budget of $250 \mathrm{msec}$. The timing result for NO_CL2 compared to CL2 ,as shown in table 9-4, shows that the time requirements are dominated by unpacking and that the logic is fast.

\begin{tabular}{|l|l|l|}
\hline mode/cone_size & time/jet (msec) & width $(\mathrm{msec})$ \\
\hline CL2 cone $=.5$ & $27+/-1.5$ & 9.0 \\
\hline CL2 cone $=.7$ & $46+/-2.2$ & 14.0 \\
\hline NOCL2 cone $=.7$ & $5 .+1-.3$ & 1.3 \\
\hline
\end{tabular}

Table 9-4 : L2JETS Timing Performance

The reconstruction quality of L2JETS is favorable compared to the full offline. The average and standard distributions of the difference between reconstructed $\eta, \phi$ and electromagnetic fraction of the jet are as shown in table 9-5.

\begin{tabular}{|l|l|l|}
\hline Offline Reco $-L 2 J E T S$ & Mean of quantity & Standard Deviation \\
\hline$\eta$ reconstructed & $.003+/-.004$ & .17 \\
\hline$\phi$ reconstructed & $.001+/-.001$ & .05 \\
\hline Em fraction & $-.022+/-.001$ & .05 \\
\hline
\end{tabular}

Table 9-5: L2JETS jet reconstruction compared to reconstruction offline

A $2 \%$ bias is evident towards lower electromagnetic fraction, but this is not anticipated to be a problem. Also the resolution is larger than the offline reconstructed resolution but is still sufficient to serve most trigger requirements. The 
distribution of L2JETS Jet ET to the offline reconstructed quantity is shown in Figure 9-8 for both the NO_CL2 and the CL2 mode. While the CL2 distribution has a peak near 1 with a width of $6 \%$, the NO_CL2 suffers from a low side shoulder which is caused by a $250 \mathrm{MeV}$ zero suppression imposed by Level 1 and the lack of calorimeter coarse hadronic information. The mean of the NO_CL2 distribution is $8 \%$ less than the CL2 distribution. Thus although NO_CL2 is many times faster than CL2, the reconstruction is poorer.

The purpose of processing jets in LEVEL 2 is to further reduce the trigger rate while maintaining efficiency. Figure 9-7 shows efficiency and rate curves obtained by requiring L2JETS to pass the event only if the calculated Jet ET is above a specified threshold. Plotted is total rate and target jet efficiency versus L2JET ET threshold for target jet ET's of 25,45 and $90 \mathrm{GeV}$. As a prerequisite, we added the Level 1 trigger proposed for that target jet $E_{T}$ in table 9-1. In all three cases, there is an efficiency plateau. Thus a L2JET cut can be chosen which maintains full efficiency while reducing the rate. Table 9-3 shows a complete set of Level 1 and Level 2 triggers for various target jets. The signal to noise ratio has been greatly improved.

\begin{tabular}{|l|l|l|l|l|}
\hline Target Jet ET & L1 Trigger & L2 Trigger & Total Rate & signal/noise \\
\hline 25 & 1 tower $>3 \mathrm{GeV}$ & 1 L2 jet $>20 \mathrm{GeV}$ & 400 & .3 \\
45 & 1 tower $>7$ & 1 L2 jet $>30$ & 60 & .28 \\
90 & 2 towers $>7$ & 1 L2 jet $>50$ & 4.5 & .08 \\
110 & 3 towers $>7$ & 1 L 2 jet $>80$ & .2 & .5 \\
155 & 4 towers $>7$ & 1 L2jet $>115$ & .03 & .1 \\
\hline
\end{tabular}

Table 9-3: Proposed Level 1 and Level 2 triggers and rates. Note that the signal rate is greatly improved over table 9-1 while full efficiency was maintained with the exception of the $45 \mathrm{GeV}$ target trigger which dropped to $96 \%$.

Although Level 1 trigger settings are sensitive to factors like noise, calorimeter resolution and the energy distribution of a jet, we expect that the Level 2 found jets will be relatively insensitive to these effects and may perform better than predicted. 
Jet Triggering Efficiency for Level 1 and Level 2
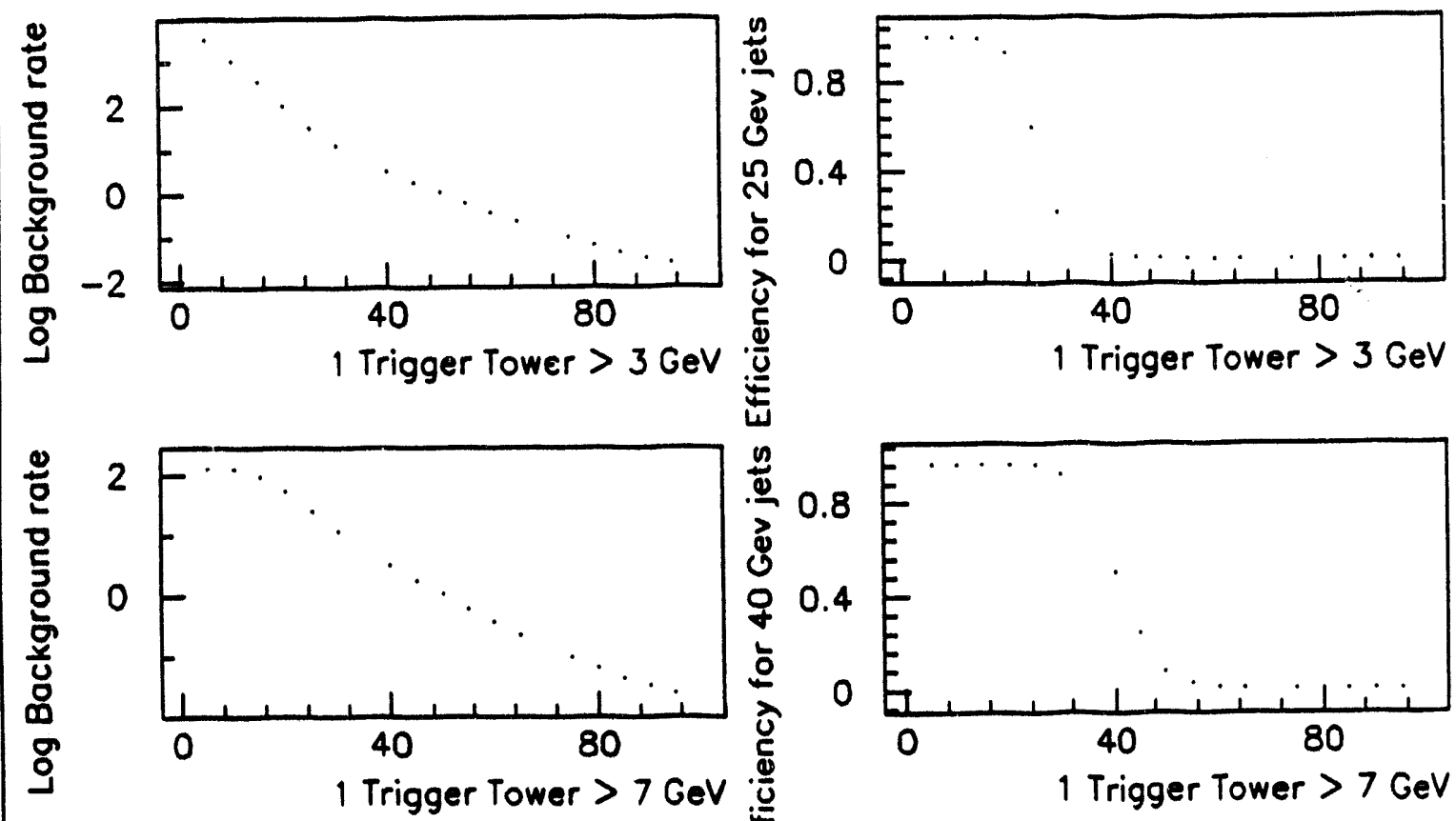

5
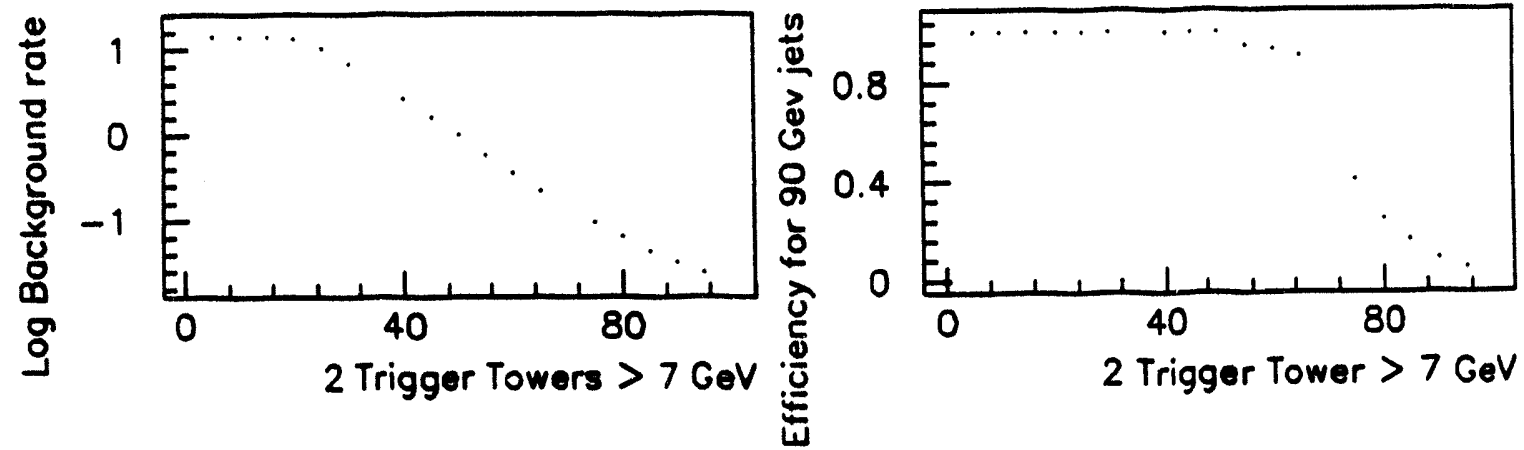

Figure 9-7:Background rotes and jet efficiencies under various Level 1 trigger settings as a function of L2JET $E_{\mathrm{T}}$ cut 


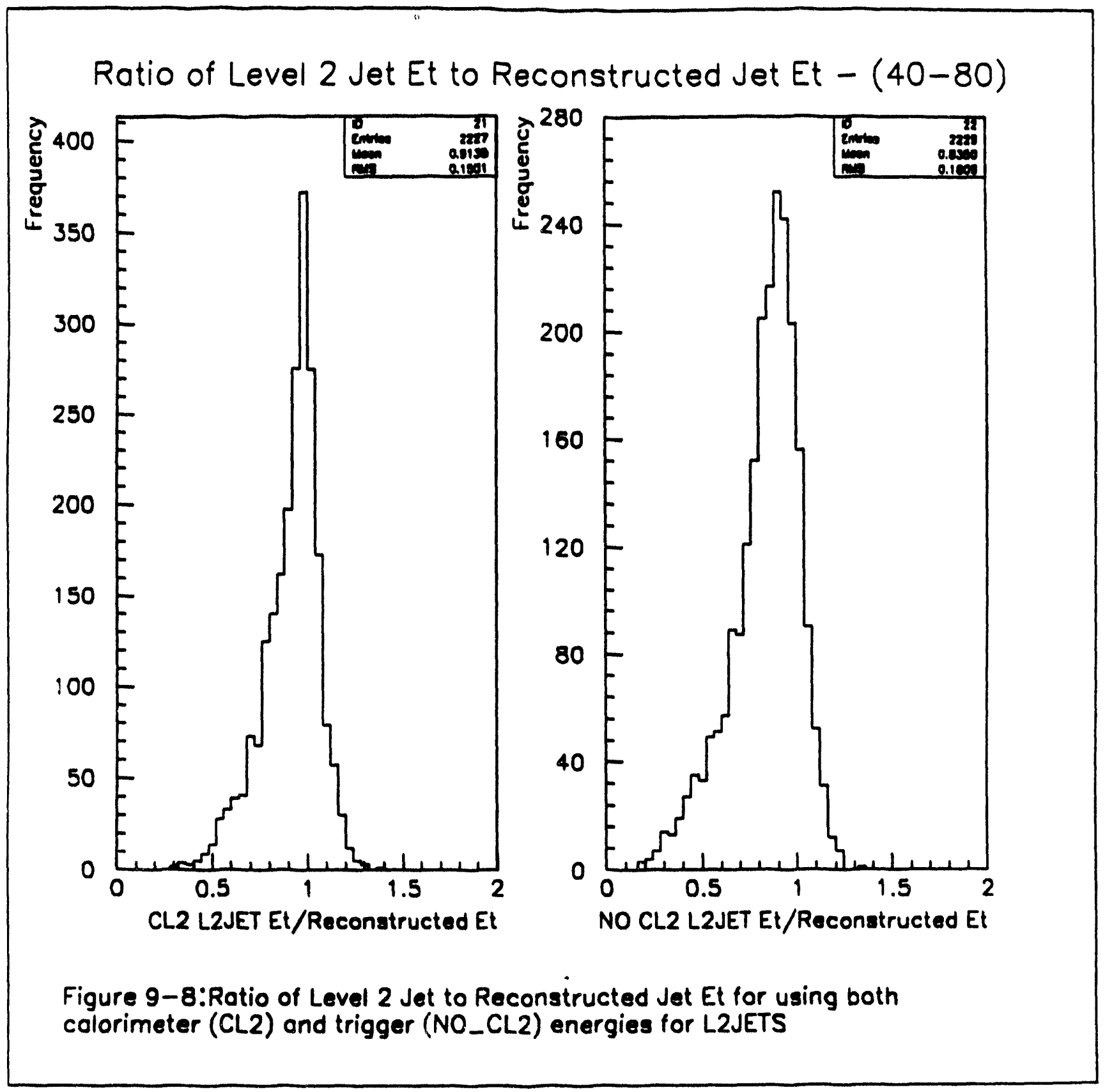




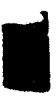

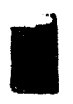

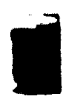

.

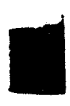

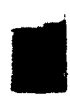

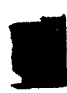

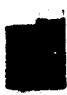

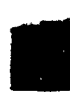

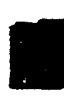

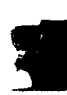

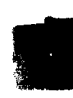

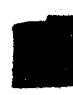

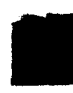

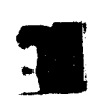
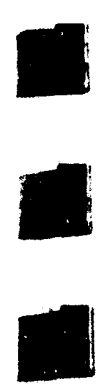
1J. Hoftun, Level 2 System Programmers Users Manual, D0 Note 909, D0 Experiment at Fermilab, Batavia, IL. December 1989.

2F. Nang and R. Partridge, Level O Detector Layout, D0 Note 998, D0 Experiment at Fermilab, Batavia, IL, August 1990.

${ }^{3} \mathrm{M}$. Abolins, D. Edmunds and B. Pi, On the Size of Hadronic Trigger Clusters, D0 Note 457, DO Experiment at Fermilab, Batavia, IL, October 1986.

${ }^{4} \mathrm{R}$. Astur and H. Weerts, Level 1 Jet Trigger and Level 2 Jet Tool: Rates and Efficiencies. D0 Note1044, D0 Experiment at Fermilab, Batavia, IL. November, 1990.

5R. Astur, Level I Calorimeter Trigger "Improvement", D0 Note 1297. D0 Experiment at Fermilab, Batavia, IL. December 1991.

$6_{R}$. Astur, Status Report on L2JETS, D0 Note 1227, D0 Experiment at Fermilab, Batavia, IL. October, 1991. 


\section{Chapter 10: Results}

In this chapter we present the procedure and results for reconstructing the single jet inclusive cross section from a large sample of Monte Carlo events. The sample used was about 100,000 events which were generated by ISAJET and processed by the detector simulator and the reconstruction software. The ISAJET events were required to have both partons from the original $2 \rightarrow 2$ interaction to have a transverse energy between 50 and $200 \mathrm{GeV}$. This does not mean that each event had a jet in this ET range as the partons are free to radiate quarks and gluons after this restriction is applied. We will use the jet reconstruction cone algorithm with cone size R- . 7 and compare to a particle PJET distribution (modified to match the Ellis definition as described in chapter 5). The PJET distribution was taken from a separate data sample of about 50,000 generated under the same conditions as the reconstructed sample. The resulting distributions from the reconstructed sample was scaled by the relative number of events (approximately $1: 2$ ) before comparing to the PJET distribution. We reconstruct three inclusive distributions versus $\mathrm{ET}_{\mathrm{T}}$ and one inclusive distribution with respect to pseudo-rapidity. The $\mathrm{ET}_{\mathrm{T}}$ was binned in steps of $5 \mathrm{GeV}$ and the $\eta$ distribution was filled in bins of $\Delta \eta-.2$. The following distributions were reconstructed:
a) $\frac{d \sigma}{d E_{T}}$
(integrated over all $\eta$ )
b) $\frac{d^{2} \sigma}{d E_{T} d \eta}(\eta=0$.) 

c) $\frac{\mathrm{d}^{2} \sigma}{\mathrm{dE}_{\mathrm{T}} \mathrm{d} \eta}(\eta=2$.)
d) $\frac{d^{2} \sigma}{d E_{T} d \eta}\left(E_{T}=57.5\right.$. $)$

Figure 10-5 shows the ratios of these uncalibrated reconstructed distributions to the corresponding input distributions. These curves would ideally be straight lines at 1.0. But mismeasurement in jet $\mathrm{E}_{\mathrm{T}}$ have diminished the reconstruction cross section to anywhere from $20 \%$ to $55 \%$ of the input value. Additionally, the shapes of the distributions have changed by $20 \%$ to $35 \%$ over the range of the horizontal axis. The following steps were taken to correct the reconstructed distribution.

\section{Correction for incorrectly merged jets}

Long after the generation and reconstruction of our high statistics sample, an error in the reconstruction code was found which directly affects the single jet cross section. As described in the DO Jet Definition chapter, the reconstruction algorithm merges two jets if the amount of energy they share is greater than a certain fraction of the energy of the least energetic of the two. This value is normally .5 , however the error in the code caused this fraction to be set at .01. Thus, some jets were merged unintentionally. As all merged jets are flagged, a procedure was set up to correct jets that were wrongly merged. Wrongly merged jets tended to have abnormally large jet widths. The reconstruction process stores results for jets with smaller cone sizes as well as the $R-.7$ size which we are discussing. The correction was based on resplitting the jets using the relative energies of the same jets which were not merged under a smaller cone size. Figure 10-1 shows comparisons of the reconstructed $\mathrm{ET}_{\mathrm{T}}$ distribution to the distribution obtained by using the correct splitting fraction, both to the incorrect splitting fraction case and for the 'corrected' distribution. A sample set of events was used to make this comparison. We can see that the distribution obtained by using a splitting fraction of .01 leads to a measured cross section that is $20-50 \%$ higher than that found when the splitting fraction is .5 . This is because smaller $E_{T}$ jets can be merged to form larger $E_{T}$ jets. When we take the sample with the splitting fraction -.01 and use the correction procedure described above, the resulting curve matches the correct one to within $10 \%$. 


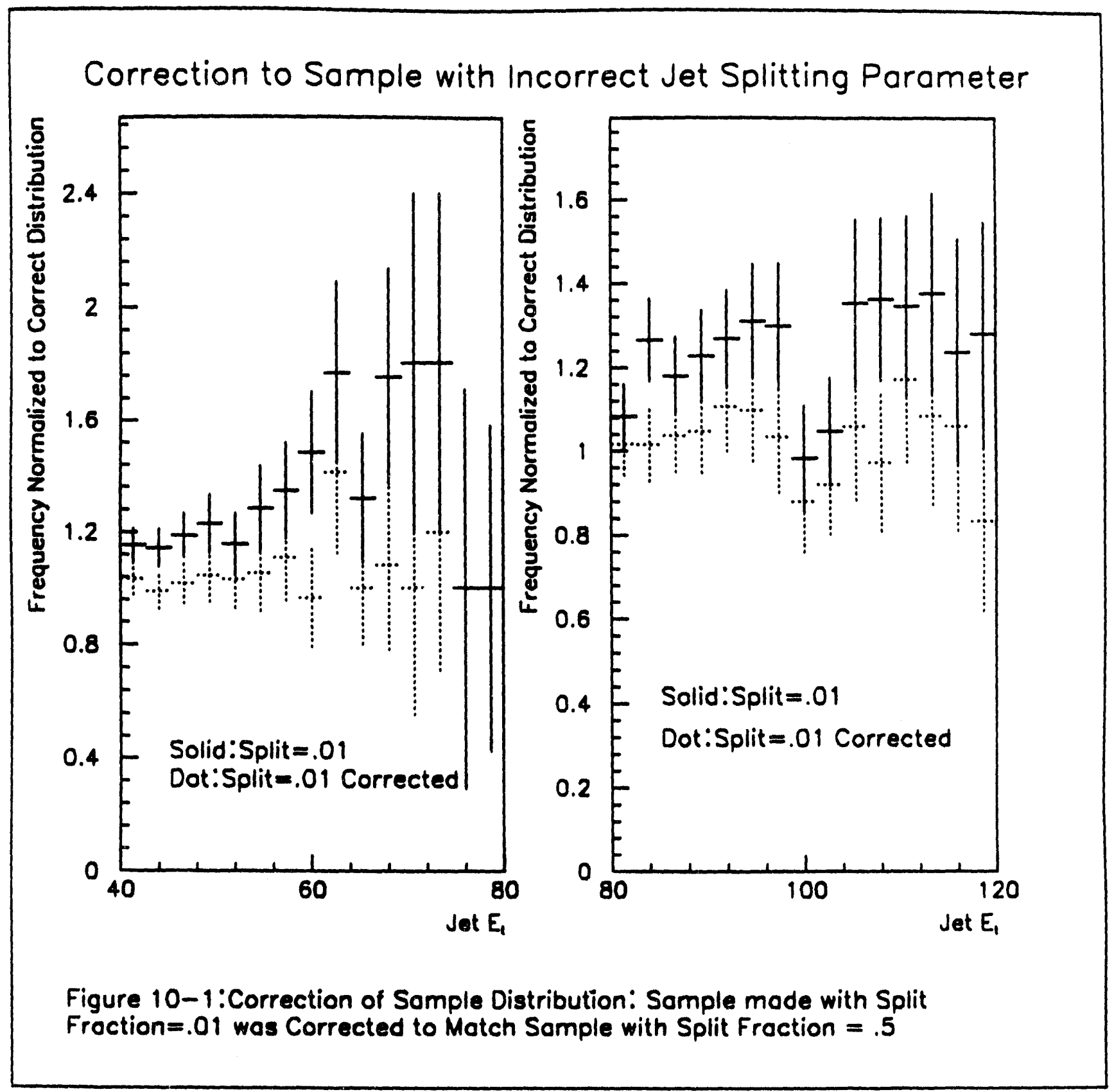

1 
Jet calibration for single particle response.

We first construct a set of predicted $R(E T)^{\prime}$ s using the third method described in the jet calibration chapter. We construct an $R(E T)$ for jets in various $\eta$ bins and two ET bins. The $\eta$ bins were 2 in size and covered $\eta-0$ to $\eta-4$. (Jets with negative $\eta$ will use the same calibration ratio as their positive $\eta$ counterparts.) Jets were generated with ET in a $40-50 \mathrm{GeV}$ and a $80-90 \mathrm{GeV}$ range and the predicted $R$ (ET) values were calculated as described in the jet calibration chapter. A comparison of these values to the real values for the $40-50 \mathrm{GeV}$ sample is shown in figures $8-18$ and 8-19. The predicted $R(E T)$ changes only by a couple percent as we move from the 40-50 GeV sample to the $80-90 \mathrm{GeV}$ sample. The falling distribution of jet ET within each $E_{T}$ bin means that the mean value is not at the center, the $R(E T)$ 's from the $40-50 \mathrm{GeV}$ and the $80-90 \mathrm{GeV}$ samples were assigned to $42.5 \mathrm{GeV}$ and $82.5 \mathrm{GeV}$ respectively. The calibration then can use a linear interpolation to determine the predicted R( ET ) for a jet with any arbitary ET. We only need to know which ET value to use and which $\eta$ io use for a given reconstructed jet. The $\eta$ of the reconstructed jet was assumed to be correct. The $\eta$ reconstruction results of chapter 7 indicate that this is a reasonable assumption, especially since our $\eta$ bins are .2 in size. ET can be approximated by using the reconstructed value of ET divided by $R(42.5)$ for that $\eta$ bin. Now the linear interpolation is done between the 42.5 and the 82.5 curve and the final calibration number is found.

\section{Out of cone showering correction.}

Our calibration did not take into account the out of cone effects seen in the forward and backward regions of the calorimeter. This correction was made by comparing the R(ET)'s of two identical samples with different cone sizes. Hence this correction explictly uses a result from the simulation. The motivation is as follows. Figure $8-6$ implies that large cone sizes are less vulnerable to out of cone energy losses. Thus the difference in the R(ET)'s between the sample with a cone size of $R-1.0$ and the same sample with cone size of $R-.7$ is a measure of the out of cone losses that are seen by the $R-.7$ algorithm, since the intrinsic response due to single particle response should be the same for both cone sized jets. This is not strictly true; one can imagine that the jets found with the larger cone size contain softer particles along their perimeter that are not present in the $R-.7$ jets. We can correct for this however by using the difference in the predicted $R(E T)$ 's for the different cone sizes. 
Figure 10-2 shows these $R(E T)$ 's for both cone sizes both as found from the simulation (real) and as predicted from the ISAJET fragmentation. We see in 10-2 that the real $R(E T)$ curves diverge for forward $\eta$. But the $R-1.0$ curve starts to flatten at $\eta$-3. implying perhaps that it is starting to experience out of cone losses as well. The predicted curves show that there is a systematic difference in the predicted response of the different cone sized jets. The smaller cone size has a $.8-1.6 \%$ greater response at all pseudo-rapidity intervals. This implies a softer particle structure in the larger cone sized jets. In any case, we must subtract the predicted differences out. Thus our $R(E T)$ 's become adjusted in the following way for each $\eta$ bin:

$$
\begin{aligned}
\mathrm{R}\left(\mathrm{E}_{\mathrm{T}}\right)_{\text {adjusted }}=\mathrm{R}\left(\mathrm{E}_{\mathrm{T}}\right)_{\text {predicted }}+\left[\mathrm{R}\left(\mathrm{E}_{\mathrm{Tt}}\right)_{\text {real }}^{\text {cone }=.7}-\mathrm{R}\left(\mathrm{E}_{\mathrm{T}}\right)_{\text {real }}^{\text {cone }=1.0}\right] \\
\\
-\left[\mathrm{R}\left(\mathrm{E}_{\mathrm{T}}\right)_{\text {predicted }}^{\text {cone }=.7}-\mathrm{R}\left(\mathrm{E}_{\mathrm{T}}\right)_{\text {predicted }}^{\text {cone }=1.0}\right]
\end{aligned}
$$

Here 'real' refers to the values actually determined in the simulation. 


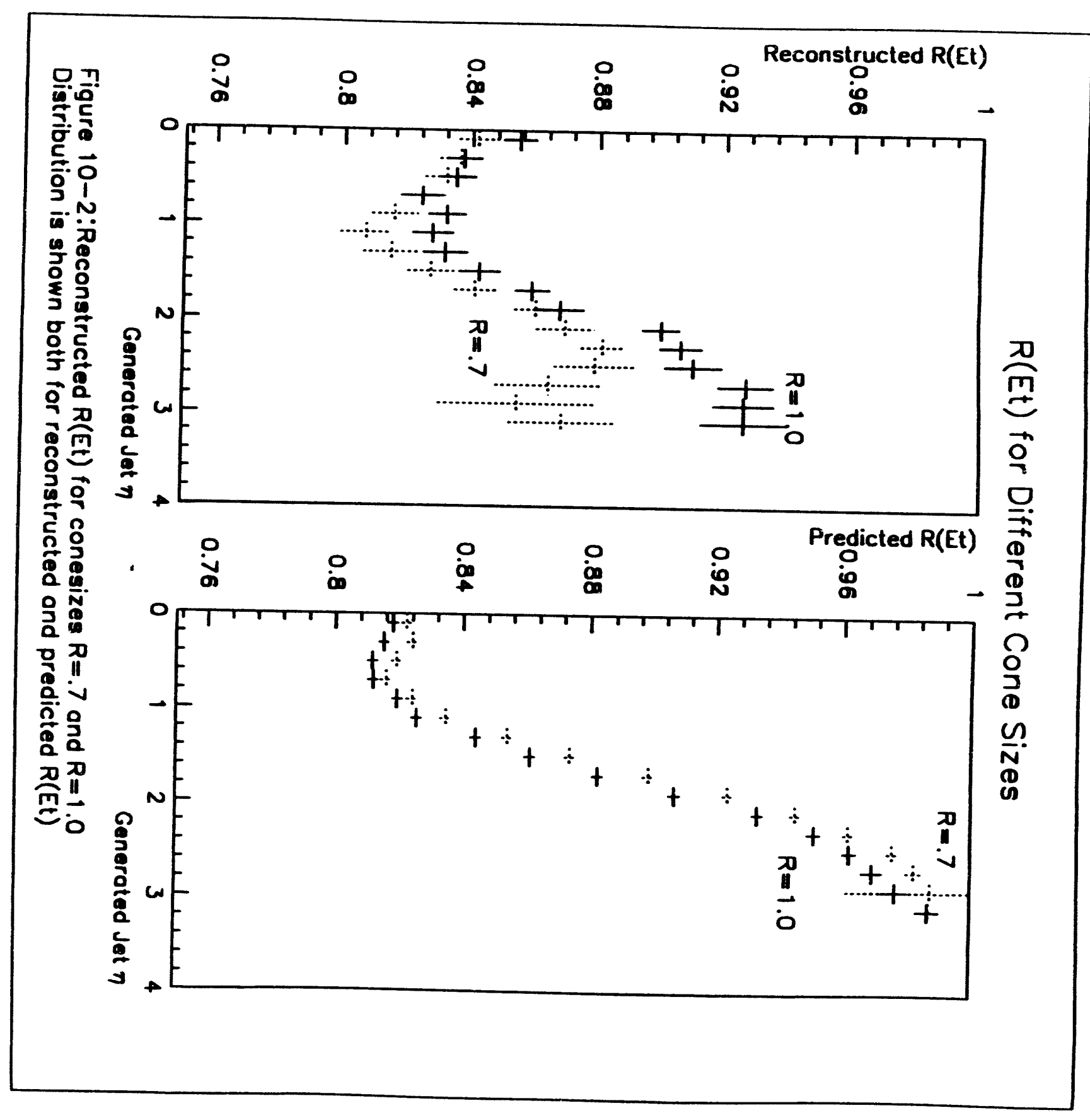




\section{Zero suppression correction.}

As mentioned before, there was no need to use a zero suppression in the simulation as there was no noise present. However, there will be one in real data taking, so the software needed to zero suppress was present. It was not used in most of the studies described in the previous chapters. However, the large data sample which we are using to reconstruct the various cross sections did have a channel zero suppression of $0.1 \mathrm{GeV}$. This means that if a cell had less than $0.1 \mathrm{GeV}$ of energy, it was set to zero. Since our calibration analysis did not have any such suppression, we must correct for this before we can calibrate this large sample. The correction term was determined by comparing the $R(E T)$ 's of a sample of jets reconstructed with and then without the $0.1 \mathrm{GeV}$ suppression. Figure 10-3 compares the two curves and shows that there was a $4 \%$ loss in energy for jets with $\eta-0$ due to the suppression. The difference of these curves was subtracted from the adjusted $R(E T)$ 's.

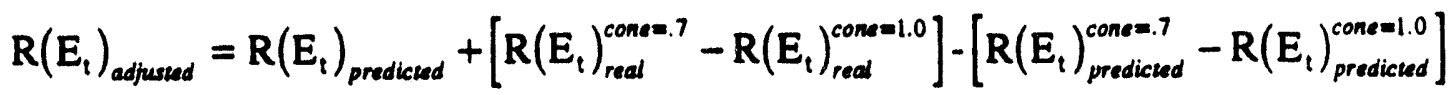

$$
\begin{aligned}
& +\left[R\left(E_{t}\right)_{\text {real }}^{1000 \text { mev zusp }}-R\left(E_{t}\right)_{\text {real }}^{\text {ao zup }}\right]
\end{aligned}
$$

\section{Final Calibration Numbers}

Figure 10-4 shows the comparison of these adjusted predicted $R\left(E_{T}\right)$ 's to the real ones we determined from the simulation. The predicted responses systematically underestimate the real response, but the forward region is much better represented than in figures 8-18 and 8-19 before the out of cone corrections were made. 


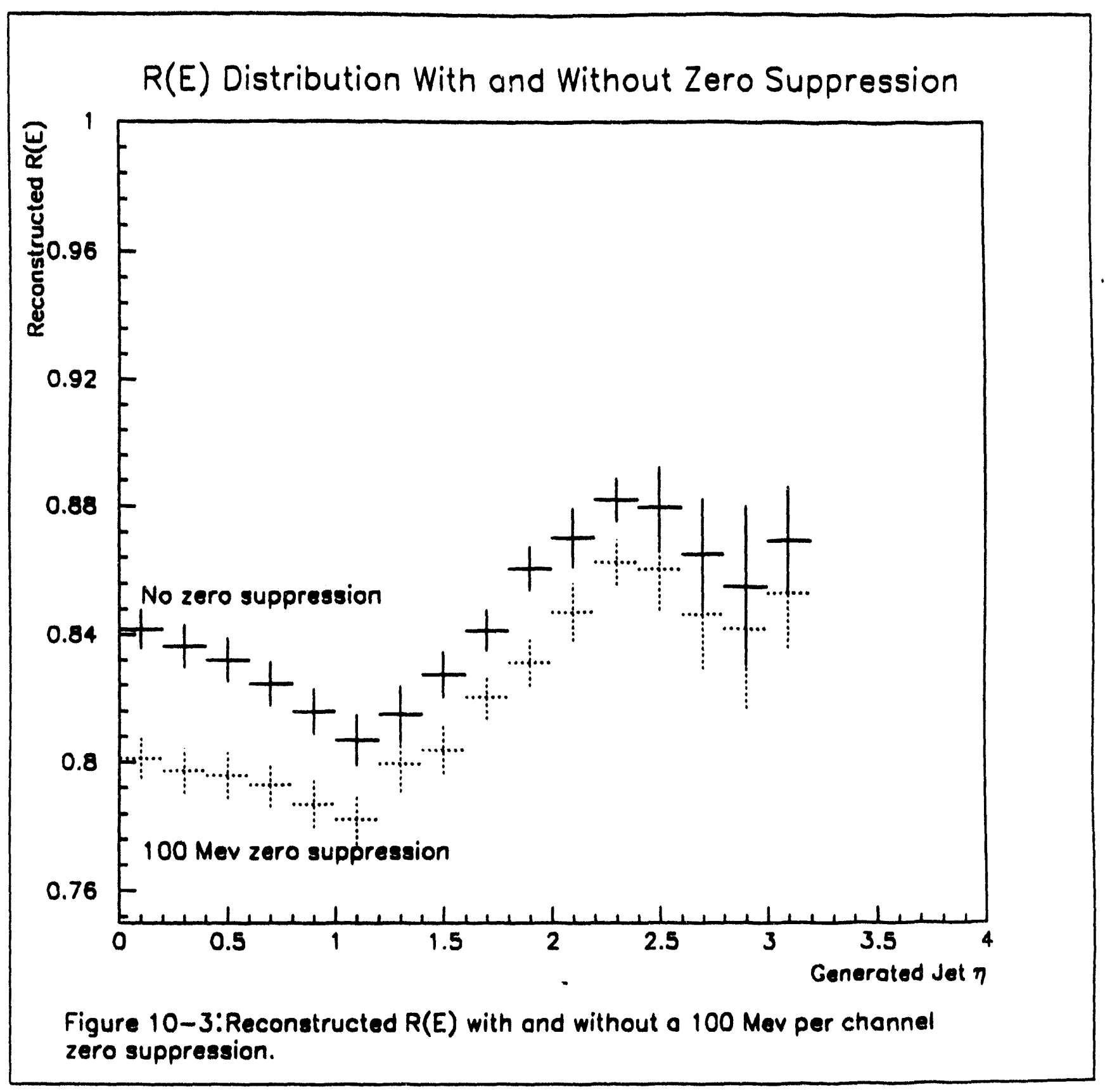


Adjusted Predicted R(Et) Distribution
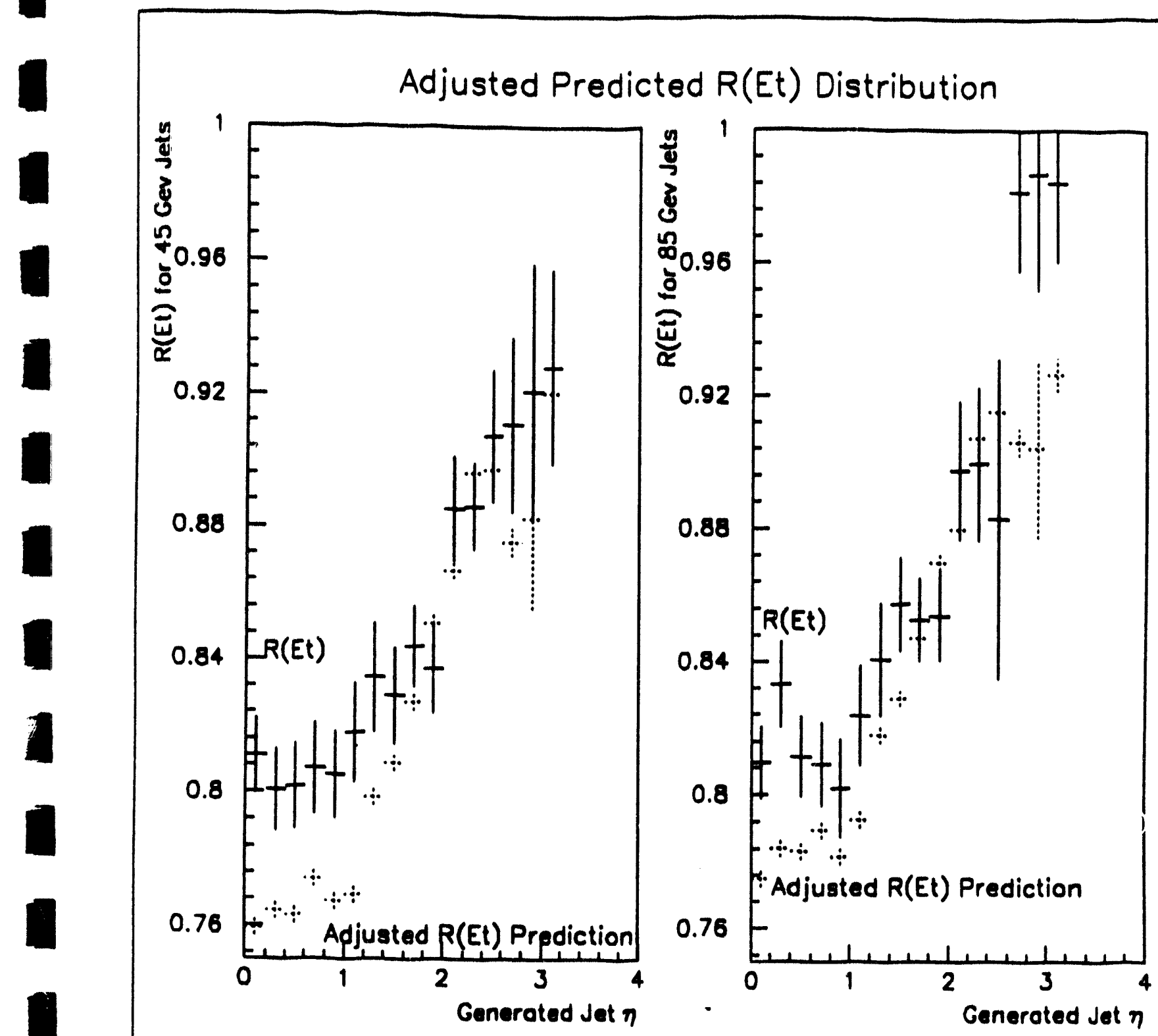

Figure 10-4:Final $R(E t)$ prediction compared with octual $R(E t)$ for jets in the 40-50 and 80-90 Gev Et bins. 


\section{Comparison of Calibrated to Generated Cross sections}

A glance at figure 10-5 is enough to see the poor comparison that the uncalibrated distributions made. We will display two sets of calibrated plots. One set will use the distributions made by calibrating with the predicted responses which we have determined. The second will use the real responses that were seen in the simulation for comparison purposes. In all cases we plot the ratio of the reconstructed distribution to the generated. Thus, ideally, the resulting curves would be horizontal lines at 1.0 Figure 10-6 shows the ratio of reconstructed distributions to the input generated distributions using the predicted responses to correct the reconstructed jets. Most points on the curves move to within $20 \%$ of the input distribution. The shape has been reconstructed to within $40 \%$ for the $\eta-0$. curve and to within $20 \%$ for the $\eta-2$. and $E_{T}=57.25$ curve. The $E_{T}$ distributions now tend to underestimate the cross section for low ET and then overestimate for the higher range of ET. This is due, to some extent, to the resolution smearing of the sample. The event sample used was generated with jet ET's between 50 and $200 \mathrm{GeV}$. Thus jets generated near $50 \mathrm{GeV}$ tended to be smeared away from the peak while higher $\mathrm{ET}_{\mathrm{T}}$ received extra entries from the lower ET portion of the distribution. We can see from figure 10-7, where the real calibration responses were used, that the same type of structure is present but not as severe. Thus the results we obtain in using the predicted responses are not so different than those we would have gotten had we used the real responses. The $\eta$ distribution tends to be reasonably flat in both. 


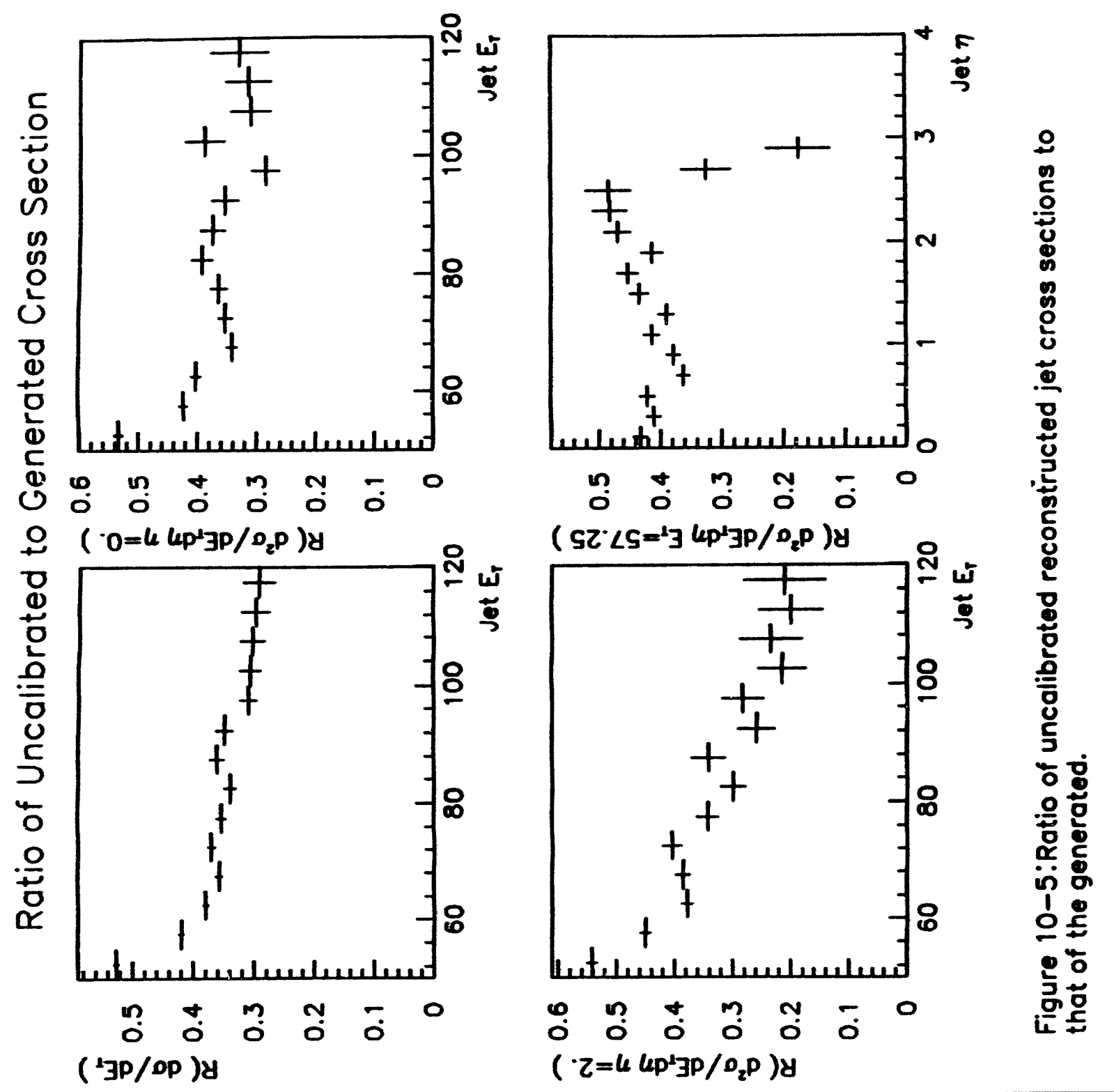




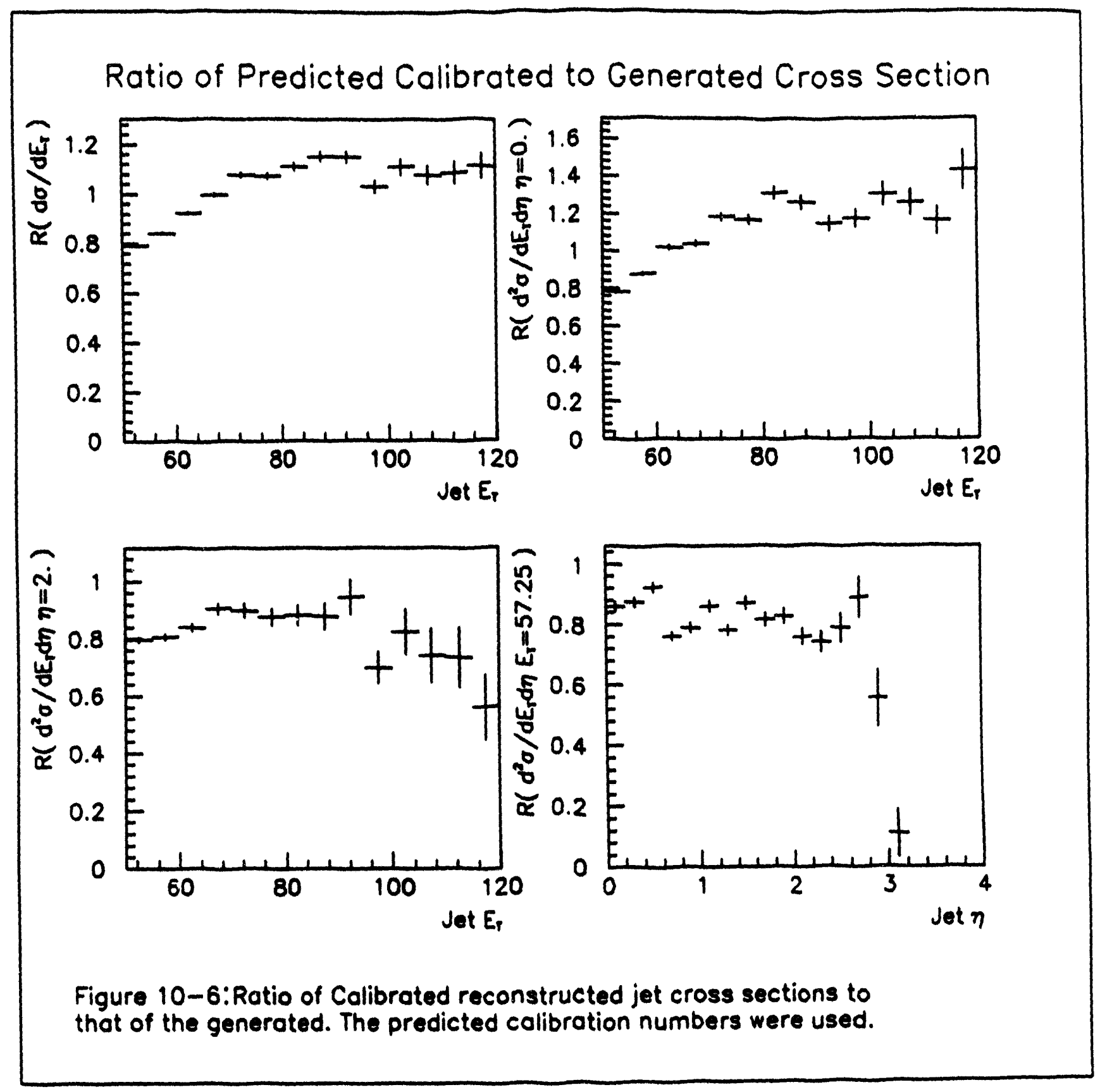




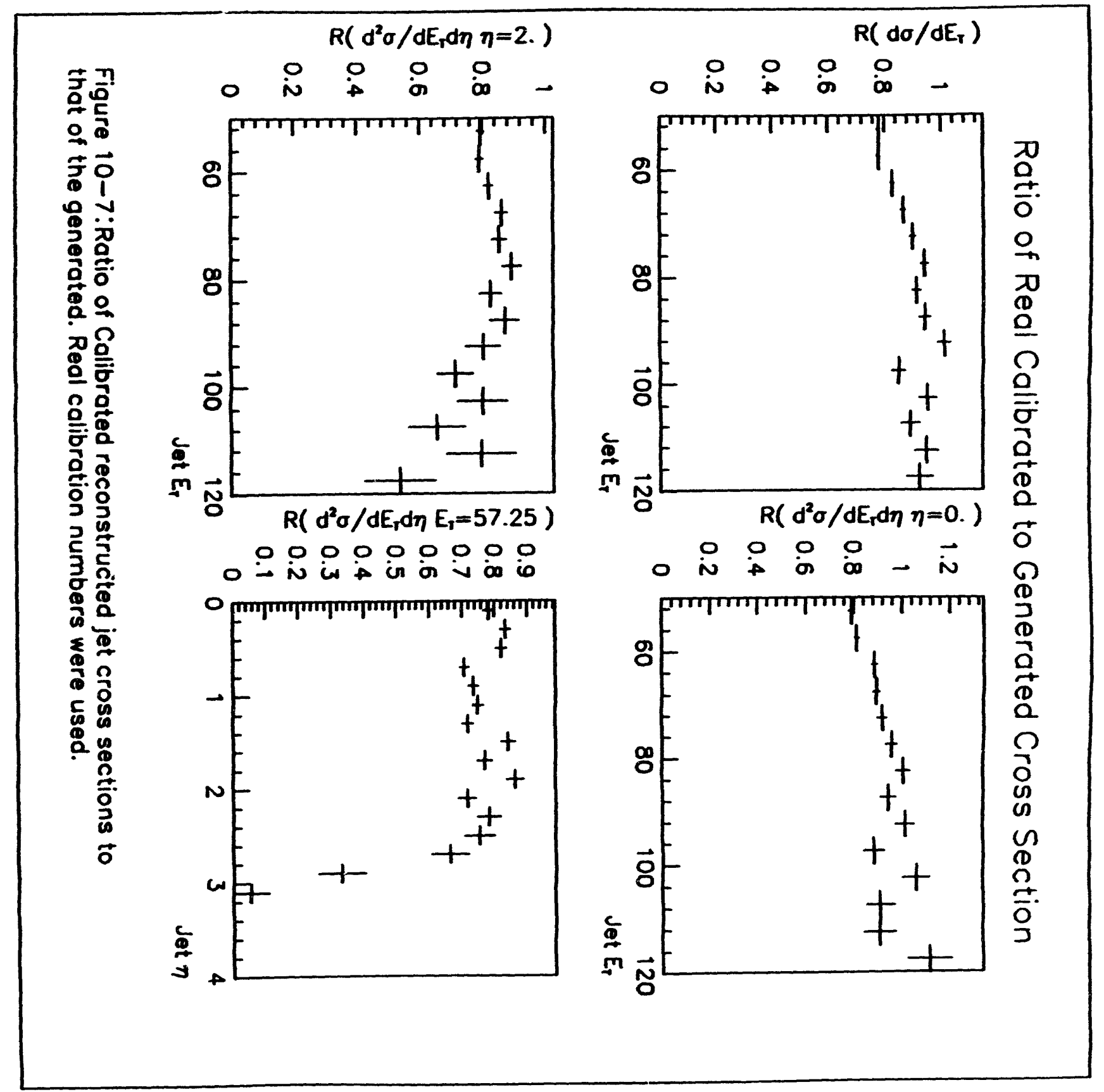




\section{Unsmearing the reconstructed distributions}

We used two methods to correct for ET resolution smearing. Both make explicit use of a simulation result. The first method is to smear the PJET distribution by the assumed jet $\mathrm{E}_{\mathrm{T}}$ resoluton before doing the comparison. This requires us to determine the ET resolution. One way to do this would be to use the resolutions of the single particle pions and electrons and convolute them with the ISAJET jet fragmentation to predict jet resolutions the same way we predicted jet responses. Doing this yields only a $6 \%$ resolution as opposed to the simulation result which is more like 10\%. Again, out of cone fluctuations probably contribute and we have not accounted for this. Although the jet ET resolution changes as a weak function of energy, we assumed it was a constant value of $10 \%$. Figure $10-8$ shows the results of smearing the modified PJET distribution before making the comparison to the predicted calibration curve. All of the curves become flatter as we expect. The shape is now reconstructed to within $20 \%$ for all the curves. Figure $10-9$ shows the same comparison but this time the real calibration responses are used. The shapes of the distribution are flat to within $10 \%$ to $15 \%$. We note that the absolute cross section is still 10-20\% less than the PJET distribution. 


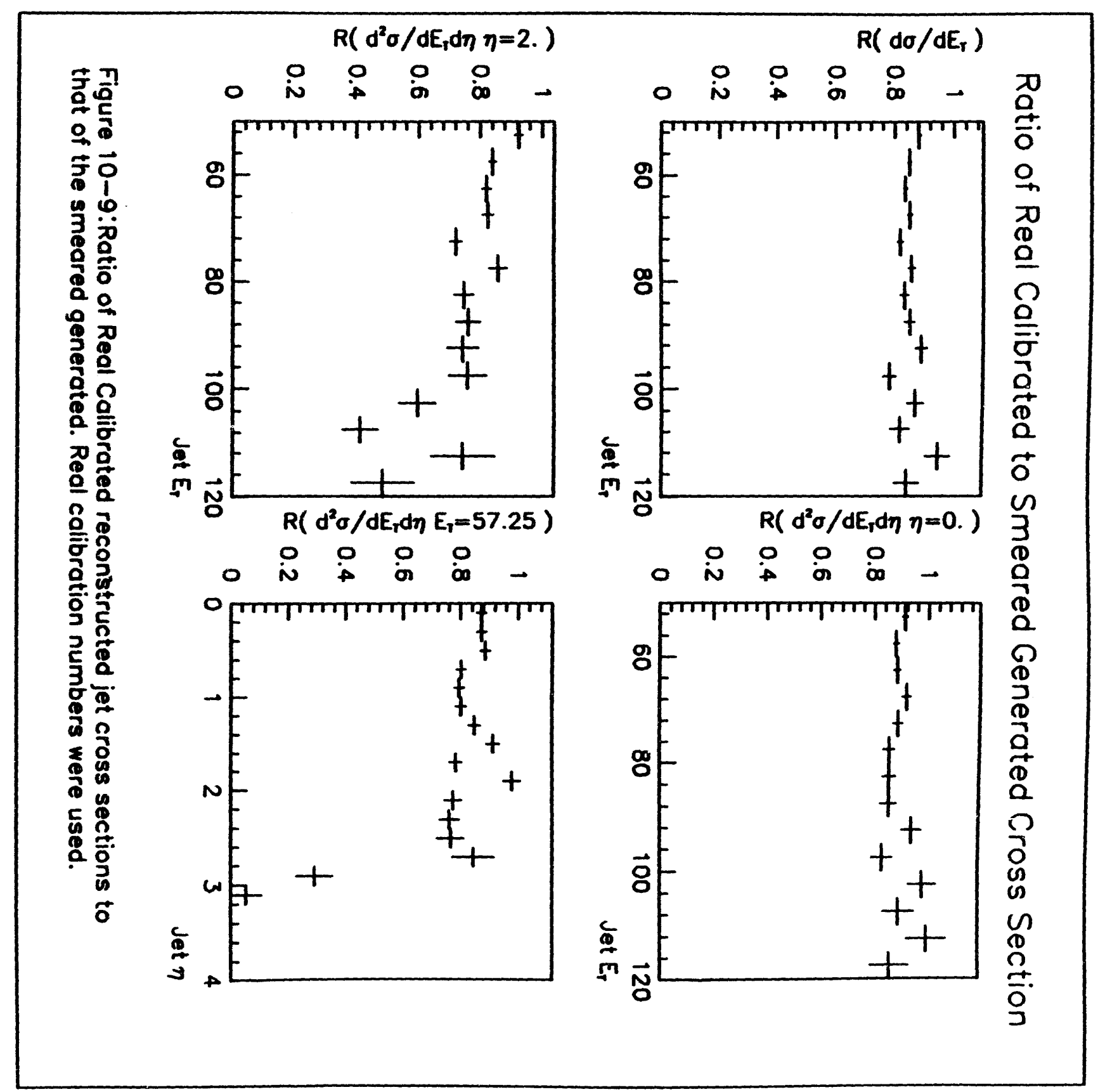


The second method used was to try to unsmear the reconstructed distribution. Again, we assume a jet ET resoluton of $10 \%$. The reconstructed distribution is smeared again using this resolution. The bin by bin difference is calculated and these differences are used to correct the original reconstructed distribution. Figures 10-10 and 10-11 show the results. In general this method does not produce a distribution that agrees with the modified PJET distribution as well as the previous method.

\section{Summary of Reconstruction}

We will summarize the accuracy of the reconstruction. The error bars in figures 10-5 through 10-11 are purely statistical. The ET distributions tend to run out of statistics for high ET so we will consider the distribution up to $100 \mathrm{GeV}$. The $\eta$ distribution runs into both statistical and out of cone problems for very forward $\eta$ so we will consider that distribution only up to $\eta-2.0$. In these regions, the overall cross section scale is reconstructed to within $10-20 \%$. The shape of the cross section is also preserved at the 15-20\% level. We can compare to this the reconstructions which used the 'real' calibration numbers. These curves reconstruct the scale to within 10 $20 \%$ but the shape reconstruction is slightly better especially for the $\eta-0$. curve. Since the real calibration numbers are used, we are led to believe that errors in jet definition and how our calibration is applied cause these $10-15 \%$ shifts. These are systematic effects. There are further systematic errors associated with errors in the ET calibration. Figure 8-16 implies that we can expect a $2 \%$ error in the predicted calibration ratios which we use. We estimate the effect on our reconstructed cross section by changing the predicted $\mathrm{R}\left(\mathrm{ET}_{\mathrm{T}}\right)$ values by $+1-2 \%$ and comparing the resulting cross section distribution to the PJET distribution. The results are shown in figure $10-17$. Thus we expect an additional $10 \%$ error in the cross section due to an assumed 2\% error in the jet $\mathrm{E}_{\mathrm{T}}$ calibration for the $\mathrm{E}_{\mathrm{T}}$ distributions. The $\eta$ distribution seems more or less insensitive and changes by only $2 \%$ with a $2 \%$ change in the $\mathrm{R}\left(\mathrm{E}_{\mathrm{T}}\right)$ values. 

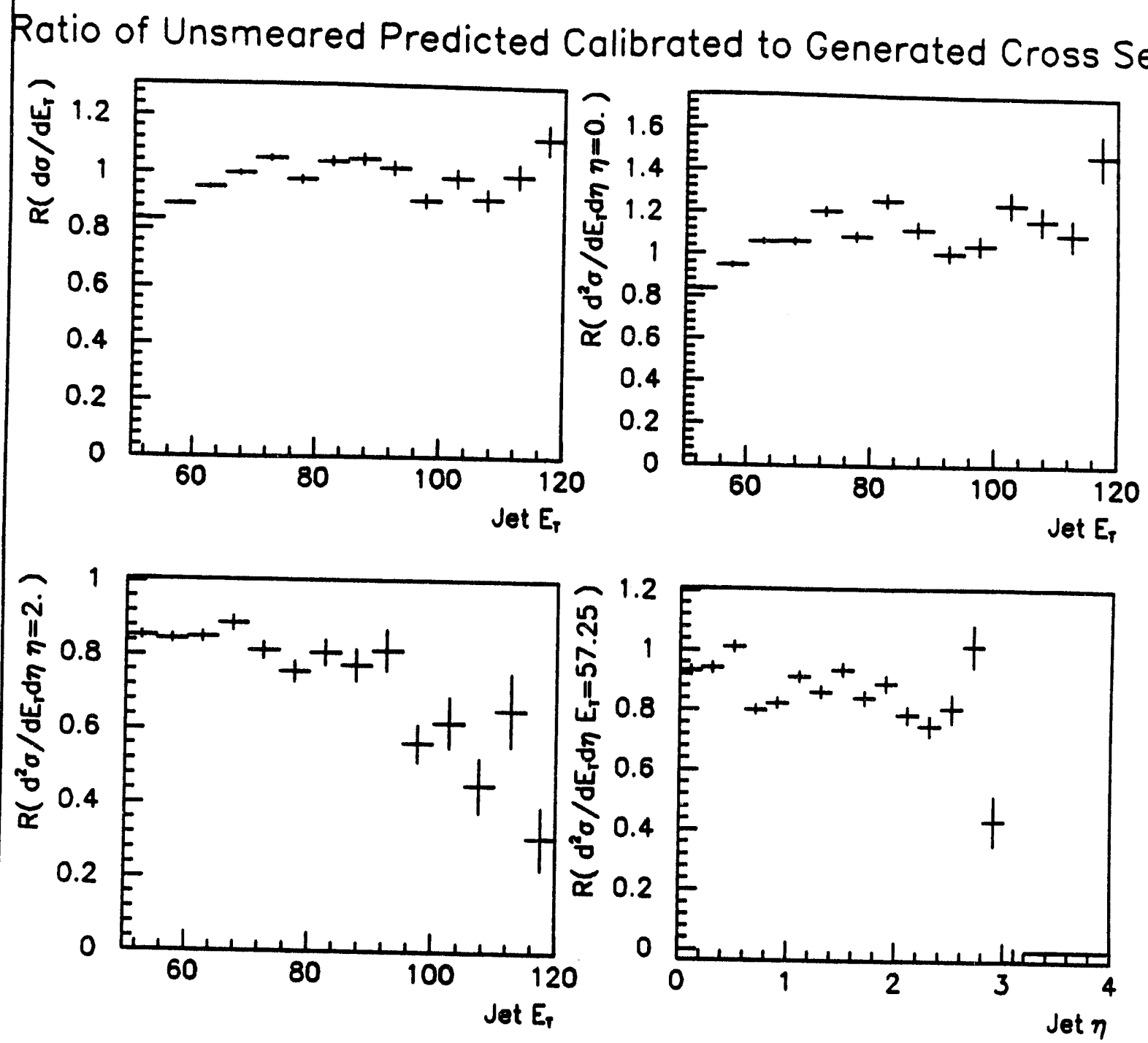

Figure 10-10:Ratio of Unsmeared Predicted Calibrated reconstructed cross sections to that of the generated. Predicted calibration numbers were used. 
Ratio of Unsmeared Real Calibrated to Generated Cross Section
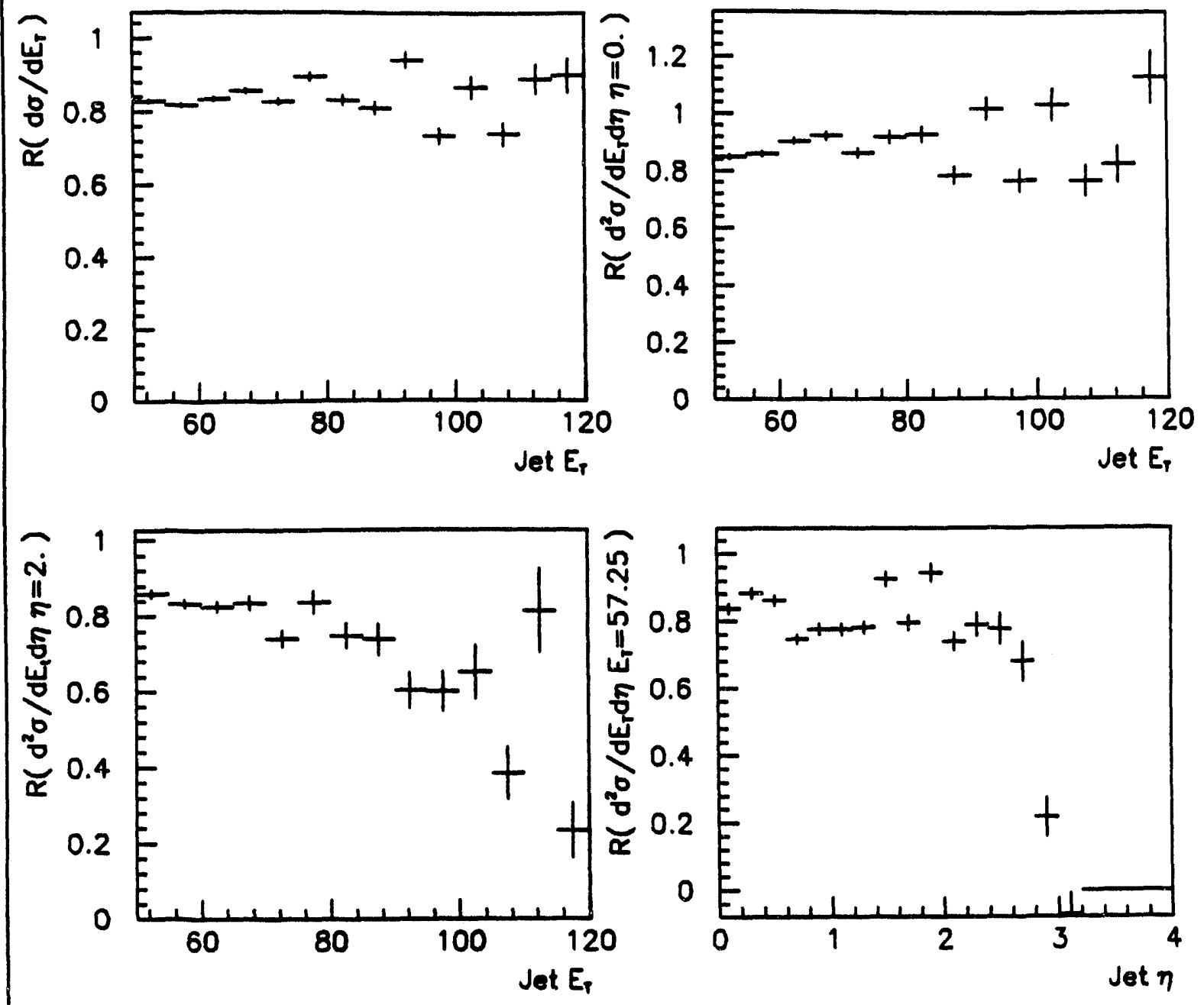

Figure 10-11:Ratio of Unsmeared Real Calibrated reconstructed jet cross sections to that of the generated. Real calibration numbers were used. 
Ratio of Predicted Calibrated to Smeared Generated Cross Section

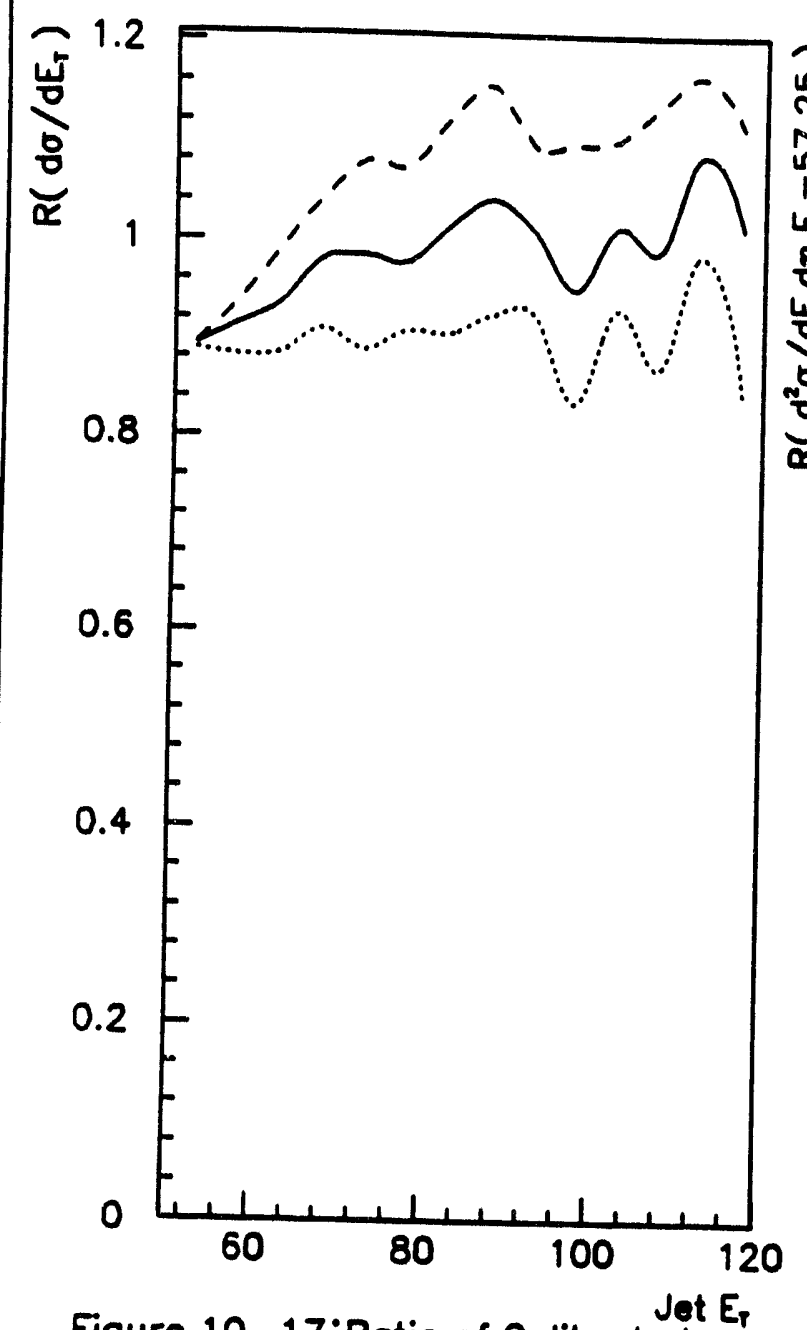

Figure 10-17:Ratio of Calibrated reconstructed jet cross sections to Jet $\eta$ that of the smeared generated. Predicted calibration numbers were Also plotted are resulting distributions when calibration is $+/-2$ percent. 


\section{Cross Section and Structure Functions}

We have seen that the next-to-leading-order predictions largely reduce the theoretical errors in the cross section prediction. However, there are still errors of order $20 \%$ from the choice of parton distribution functions. A precision measurement of the single jet inclusive cross section could be compared to several NLO theoretical predictions generated using various sets of parton distribution function. In doing so, it may be that some sets would be favored over others and thus the parameters used to generate the p.d.f's could be further constrained. ${ }^{1}$ We will investigate the difference in the predicted cross sections obtained by using various sets of structure functions calculated by Martin, Roberts and Stirling. These sets were calculated in the same way except for two parameters. The first is the QCD $\triangle$ parameter. The second parameter is $\eta_{\mathbf{g}}$ which has to do with the gluon parton distribution function which typically has the form:

$$
g(x)=A x^{\lambda}(1-x)^{\eta_{1}}
$$

The best fit of these two parameters was called B0. Four more fits were created by choosing 4 points along a chi-square contour in $\Lambda-\eta_{\mathrm{g}}$ space. The chosen parameters and their labels are shown in table 10-1.

\begin{tabular}{|l|l|l|}
\hline Set & $\Lambda^{(4)} \mathrm{MeV}$ & $\eta_{g}$ \\
B0 & 190 & 5.10 \\
B135 & 135 & 4.65 \\
B160 & 160 & 4.25 \\
B200 & 200 & 5.65 \\
B235 & 235 & 5.20 \\
\hline
\end{tabular}

Table 10-2: Parameter values for various parton distribution fits.

Figure 10-12 shows the cross sections predicted by using each of these sets when normalized to the B0 fit. The distributions differ by as much as $20 \%$ in certain PT ranges. Changes in $\Lambda$ tend to cause normalization changes while changes in $\eta_{g}$ changes the shape. Figure $10-13$ is the same plot but at $\eta-2$. Like figure $10-12$, the high and low PT regions are where there is the largest difference between the curves. Note that there are both scale and shape differences. Figure 10-14 is the same type of plot but this time the $\eta$ cross section at fixed $\mathrm{PT}-10$. GeV is used. The differences are mostly in the scale, but they are large differences. This is at very low PT, however; figure 10-15 shows the results for a PT-60. GeV point which is closer to the sample we are using. But here the differences are much less. So we see that the predicted 
cross section distributions vary widely in different kinematic regions, but the sample we are using seems to be in one of the kinematic regions where the differences are not as large. 


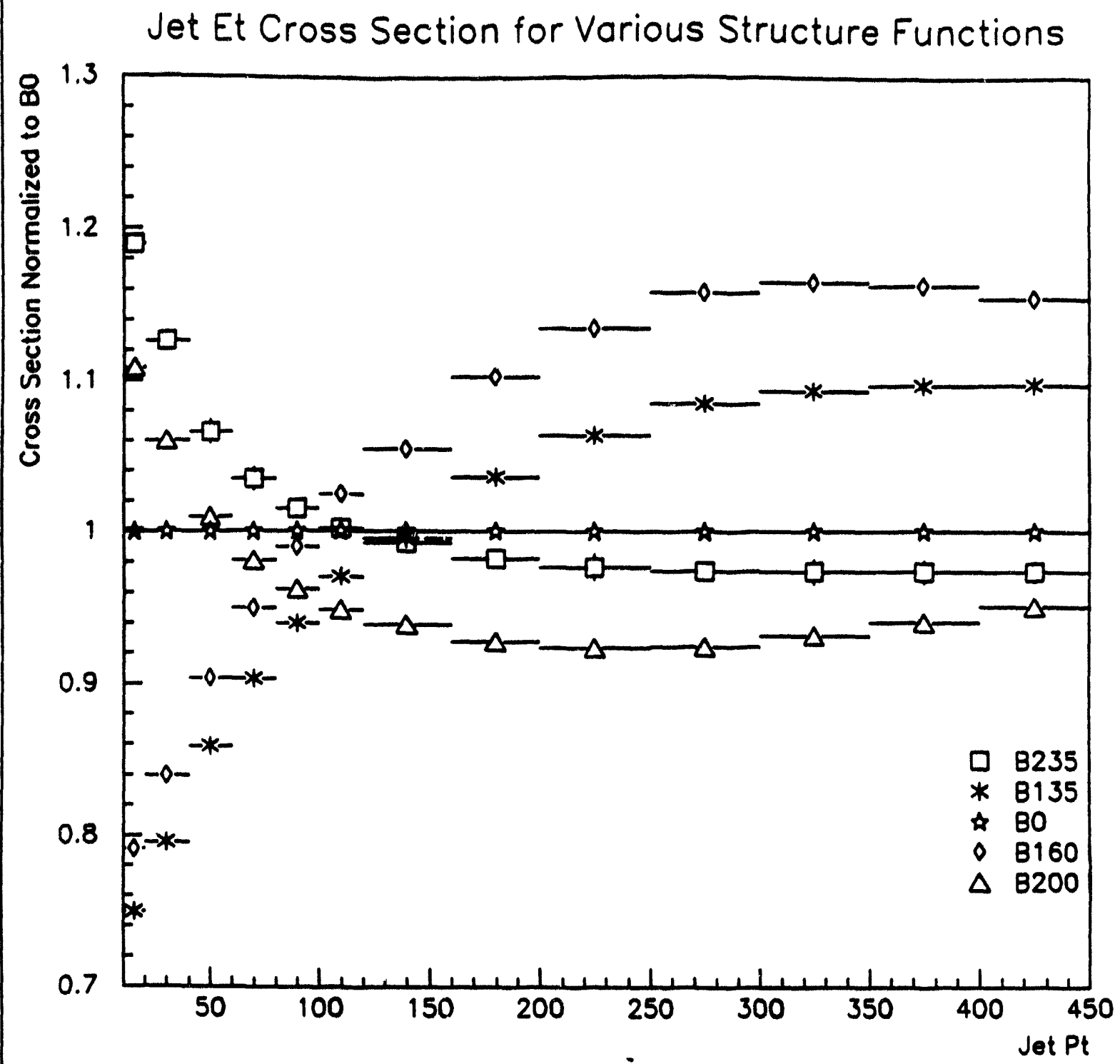

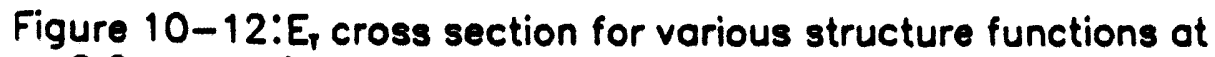
$\eta=0.0$, normalized to $\mathrm{BO}$ 


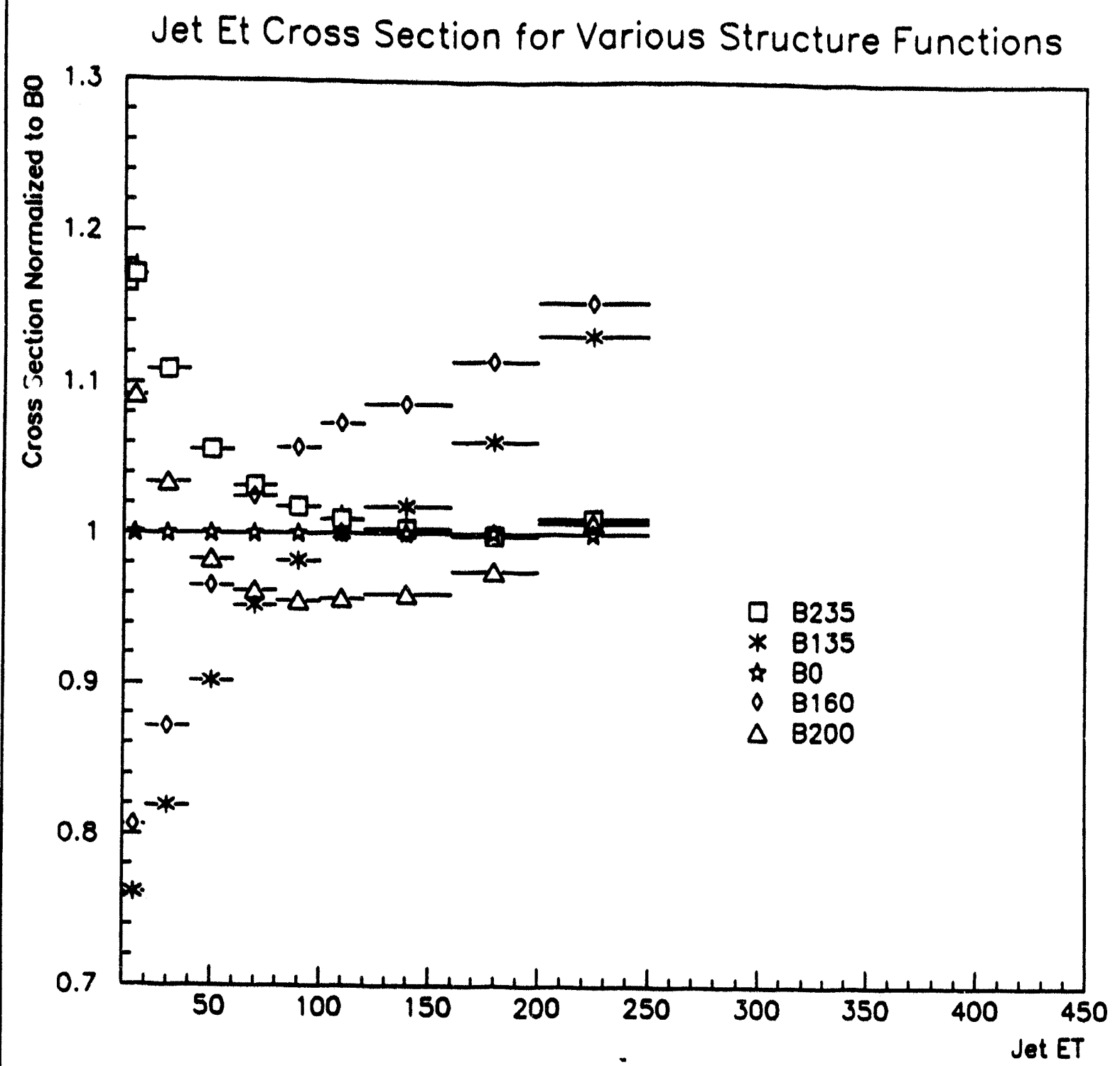

Figure 10-13:LO E, cross section for various structure functions at $\eta=2.0$ normalized to $B O$ 


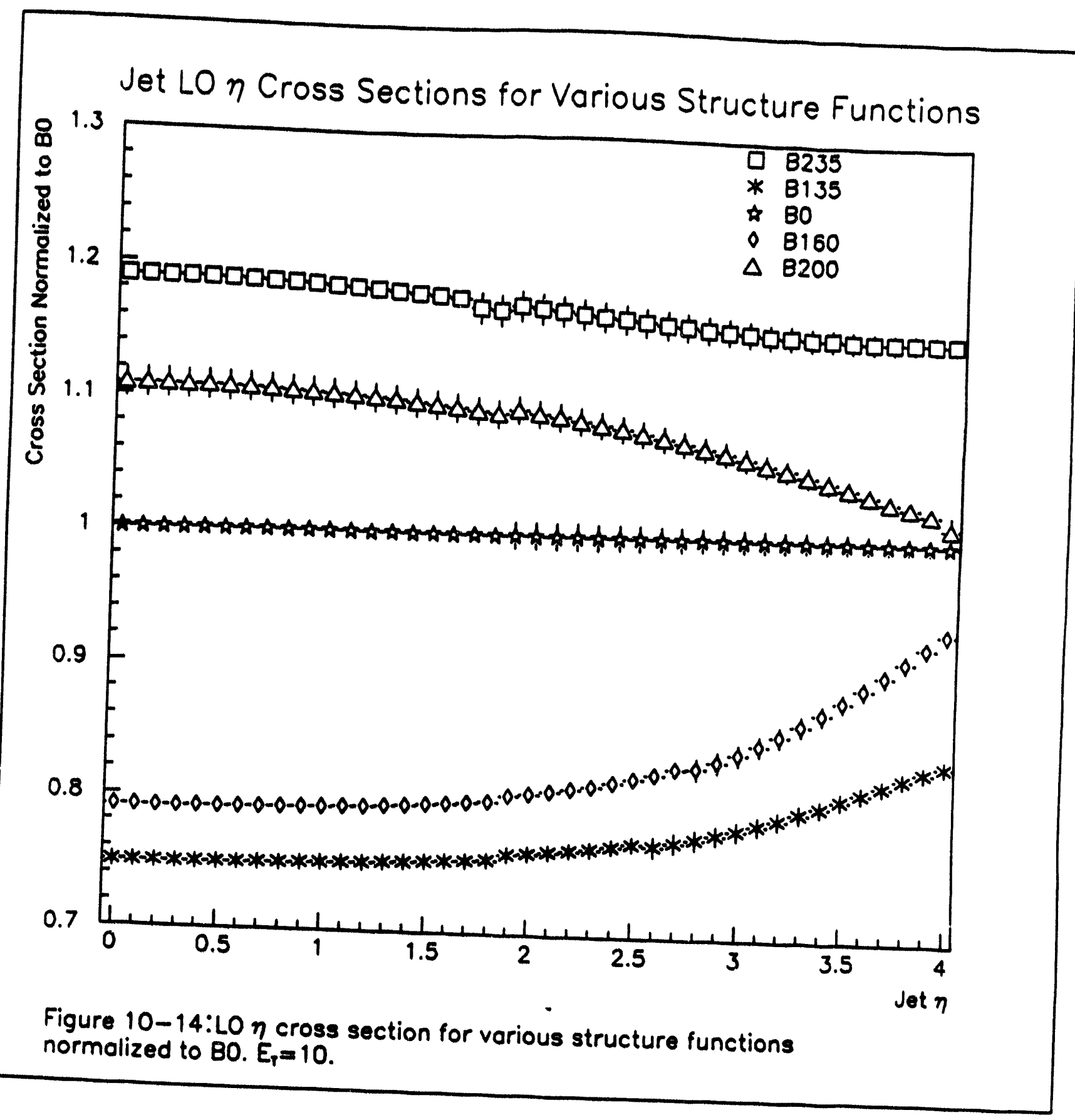




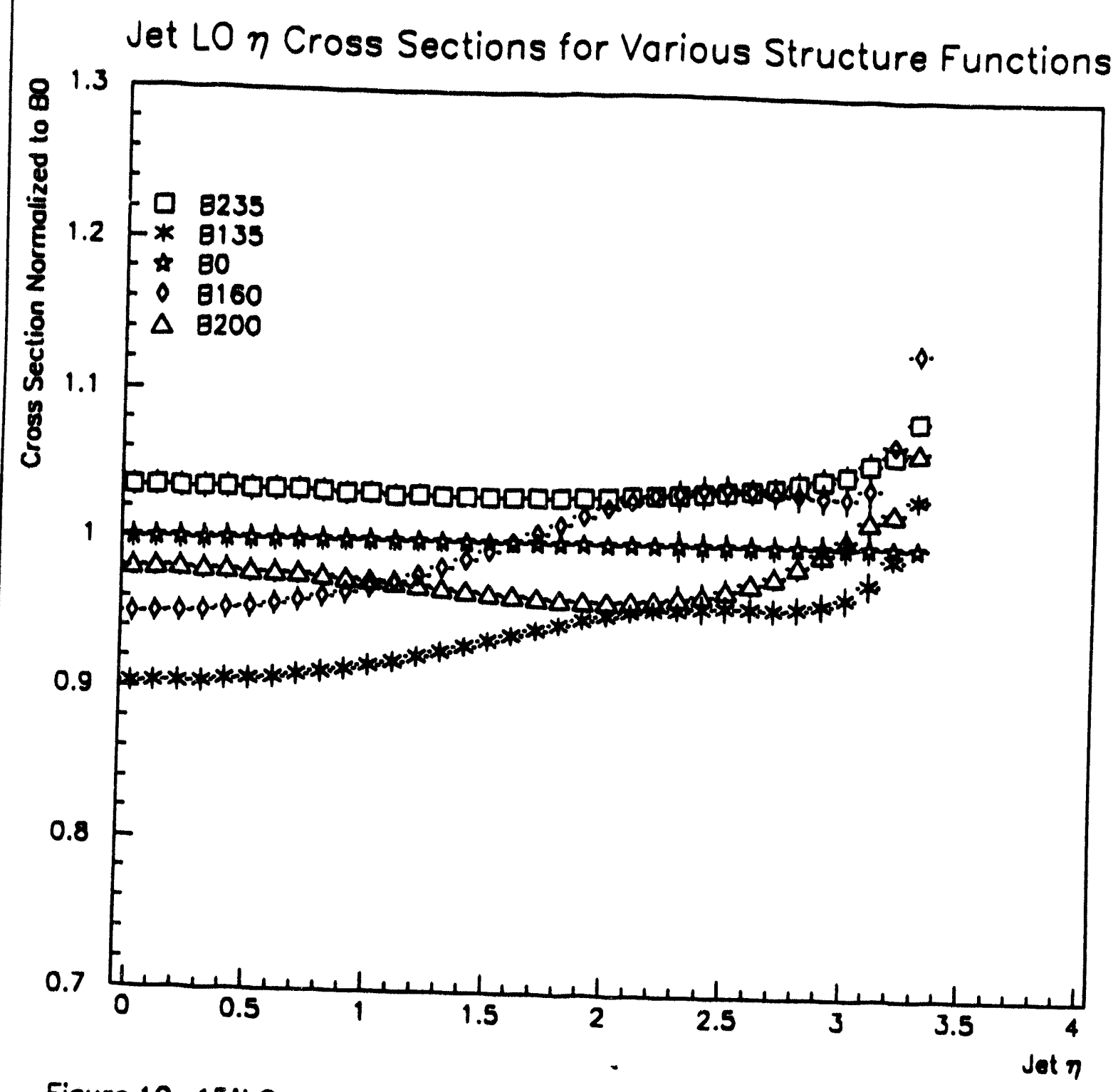

Figure 10-15:LO $\eta$ cross section for various structure functions normalized to $B 0 . E_{1}=60$. 
Figures $10-12$ through 10-15 were generated using LO predictions only. It is important to know if the NLO predictions show the same, less or more dependence on the choice of parton distribution functions. The answer seems to be that the dependence is the same. Figure 10-16 shows one prediction for both LO and NLO as an example of this.

If we were able to reconstruct the cross section precisely enough, we would be sensitive to the normalization and shape differences between the different sets. The above plots seem to imply that one must be sensitive to $5-10 \%$ differences in the cross section. In the range of our reconstructed sample, even betier precision is necessary. The reconstrurtion results show that the reconstructed distributions are uncertain by $20-25 \%$. In figure 10-18 we have superimposed the ratio of our reconstructed cross section to that of the input PJET distribution to the other structure function ratios. The reconstruction is not nearly precise enough to differentiate among them. 
Jet $E_{T}^{P}$ Cross Sections for Various Structure Functions: LO vs NLO
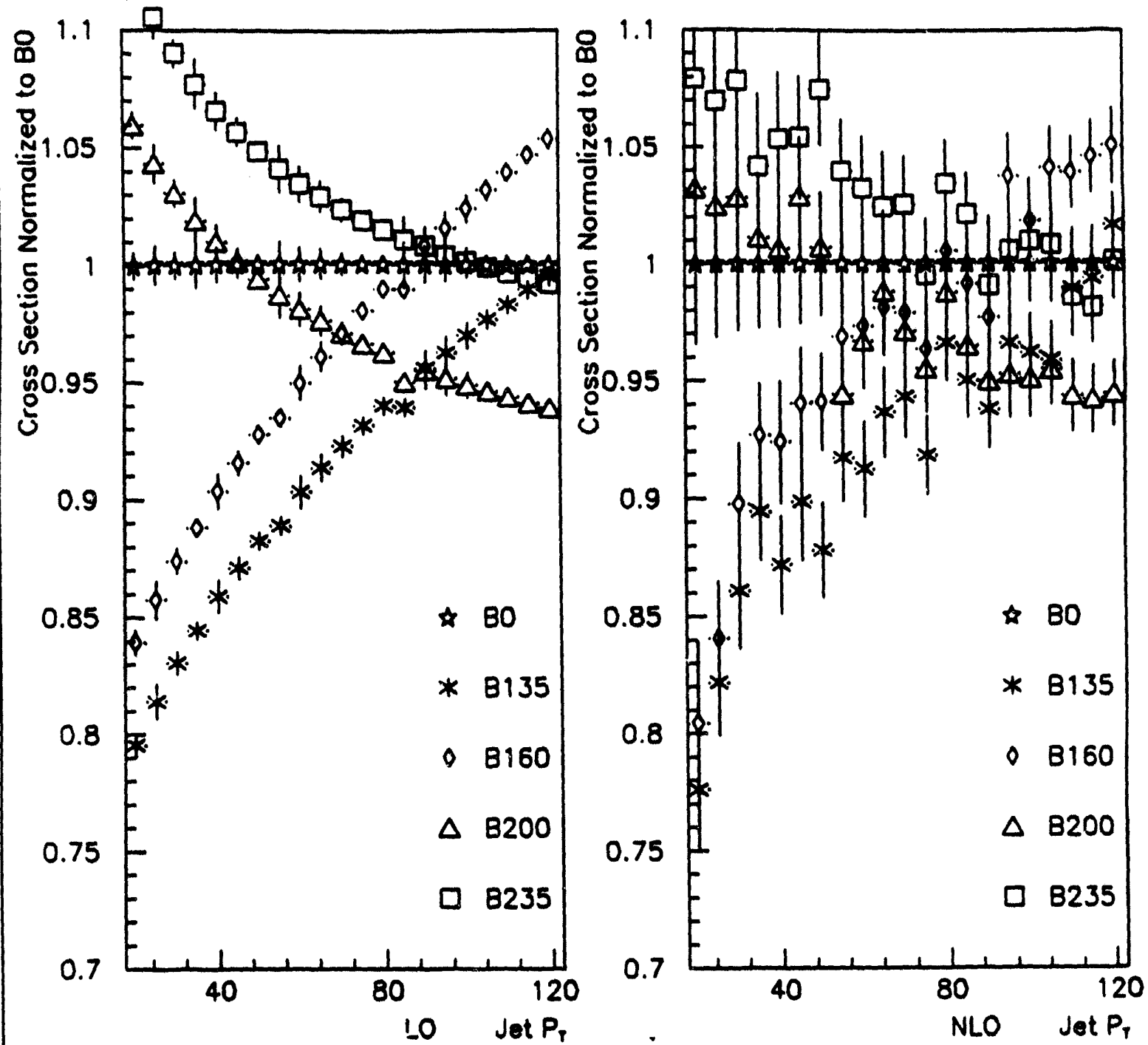

Figure 10-16: Et cross section for various structure functions at $\eta=0$. normalized to $B O$. LO versus NLO. 


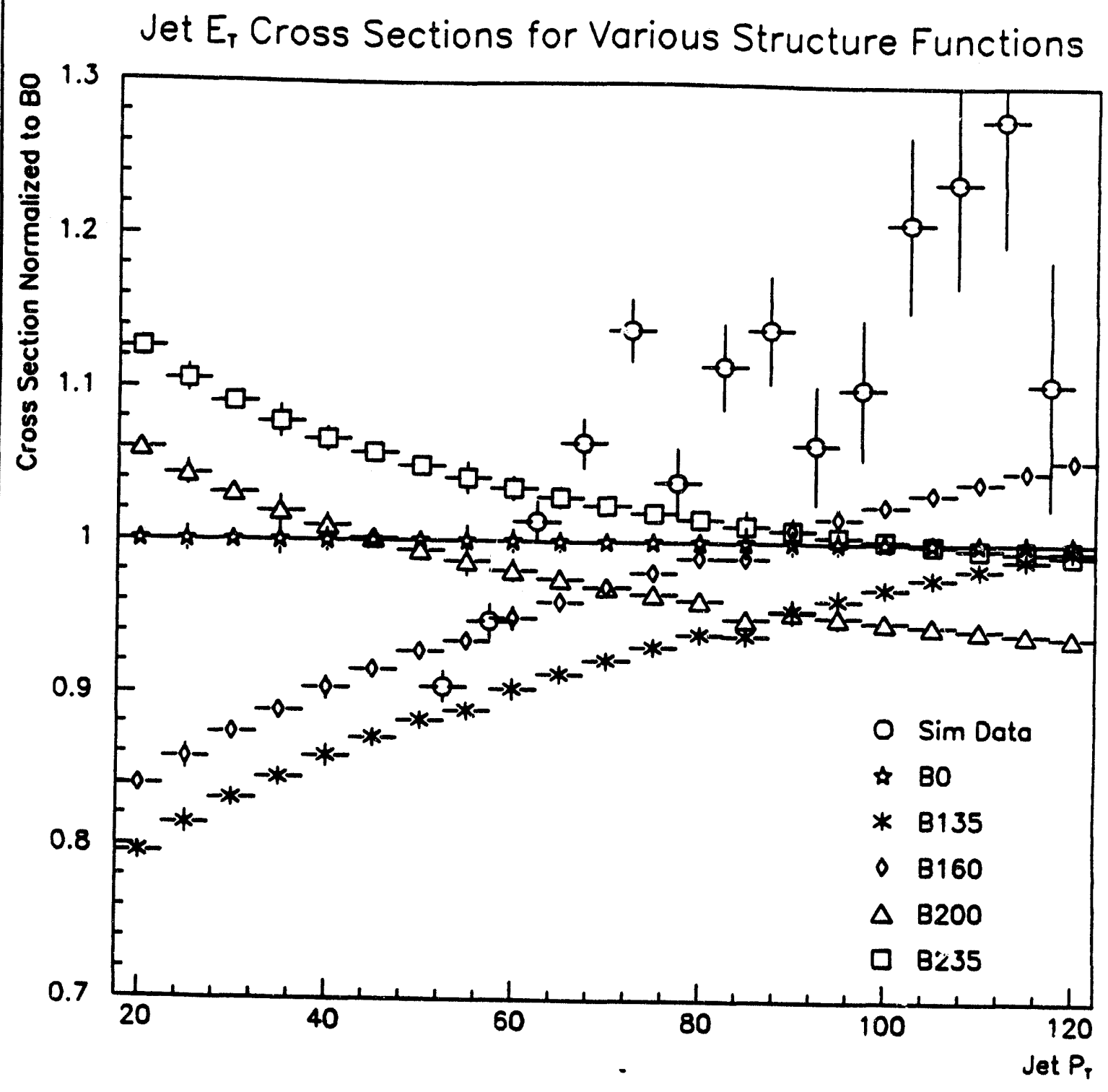

Figure 10-18: Et cross section for vorious structure functions ot $\eta=0$. normalized to BO. Dato points are normalized to modified PJETS 


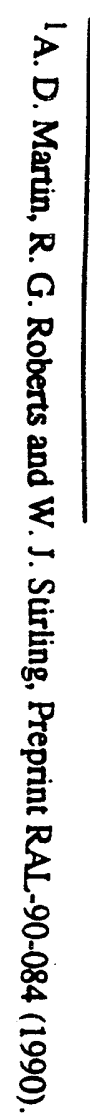




\section{Chapter 11: CONCLUSIONS}

The previous sections are the resuits of simulations and studies done so far and are by no means complete. It may be useful to summarize some of the main issues raised along with a general indication of how well it is believed to be understood.

1) Many of the above sections as well as the appendices emphasize the importance of understanding the definition of a 'jet' when attempting to reconstruct the inclusive cross section. This analysis focused for the most part on the Ellis prescription with a cone size of .7 radians. Other definitions may produce different results. It has been implied here that the standard D0 definition of parton jets (PJETS) is not compatible with the Ellis definition and that the D0 reconstructed definition of jets may indeed be compatible with the Ellis prescription. But it was also clear that they are not identical and the effect of their differences on the measured cross section are not understood. For use in comparing to NLO theory, it would seem to make sense to study how these algorithms can be tuned or modified to maximally respect the theoretical definition.

2) D0's ability to reconstruct jets over a wide range of $E_{T}$ and $\eta$ space seems promising but will be very dependent on the quality of the energy calibration. The calibration method used here is simple and can be improved in many ways. The jets should be calibrated on the basis of the location of the detector in which they were found and not based on the $\eta$ of their jet axis. Further, we have seen that a 'jet' may consist of several subclusters of energy which represent different partons. Calibration should be applied on the basis of these clusters instead of on the entire jet. Finally, out-of-cone losses threaten to be a very important phenomena for both forward jet physics and for studies with different cone sizes. We must learn to separate energy that belongs inside 
the cone from energy that belongs outside the cone. This may require changing the fixed cone into a more flexible object which depends on rapidity and the center of mass trame of the scattering.

3) This paper deals, for the most part, with the reconstruction of the cross sections of the particle jets that are created by the underlying parton jets. There is a clear fragmentation dependent difference in these two distributions and no attempt has been made here to recreate the partonic distributions which would ideally be compared with theory.

4) Energy calibration seems to be dominated by the effects of single particle responses in the calorimeter. A surprisingly good correction can be made by determining the single particle responses and combining this with an assumed fragmentation. Hence, careful analysis of the linearity of pions and electrons in DO's fixed target test calorimeter is crucial in supplying a first order jet energy correction at collider start up. This correction can be used both for offline analysis as well as online triggering and display. Such analysis and work is underway.

5) In order to be able to make statements about structure functions via measurements of the single jet inclusive cross section, the precision of the reconstruction must be improved over what has been shown here; additionally, any such attempt must consider the low and high jet PT regions where the cross section is most sensitive to the structure functions. 


\section{APPENDIX A: Level 1 Trigger Sensitivity}

Efficiencies and rates have been calculated for various $\mathrm{E}_{\mathrm{T}}$ jets for various trigger settings. It is expected, however, that these numbers will be sensitive to differences between the simulation and the real detector. For example, noise would tend to increase trigger rates, while wider jets would decrease trigger efficiency. We have studied the effect of the Level 1 trigger to the different calorimeter resolutions, noise , and different event generators.

\section{Sensitivity to Event Generator.}

Sensitivity to the event generator was done by comparing ISAJET to HERWIG. CDF has found that HERWIG agrees very well with the fragmentation dependent quantities they are able to measure with their central tracking detector. (Unlike D0, they are able to determine the momentum of charged particles by using their central magnetic field and measuring the curvature of the particle's trajectory.) Both simulations were run to produce jets between 50 and $200 \mathrm{GeV}$. The final state generated jets were defined by looking at the particles produced, applying a cone of $R=.7$ and requiring that there be at least $8 \mathrm{GeV}$ in order for the jet to counted. Five hundred events were generated in each case. ISAJET generated 2921 jets and HERWIG generated 2848 jets. The results were placed into a toy DO calorimeter, no energy cutoffs were made, the energies were smeared with the calorimeter resolution and longitudinal showers were simulated. NOTE: this is NOT the same as the much more sophisticated and detailed DOGEANT detector simulation. Thus effects like $e / \pi$ 
and energy linearity will not show up in this comparison. But we should see effects of different event topology and jet energy distribution. The $\mathrm{E}_{\mathrm{T}}$ distributions looked similar. Table AA-1 shows the jet ET breakdown of these events.

\begin{tabular}{|l|l|l|}
\hline Jet Et Bin & Herwig & Isajet \\
\hline $0 \_5$ & $30 \%$ & $18 \%$ \\
$5 \_10$ & $16 \%$ & $22 \%$ \\
$30 \_35$ & $2.8 \%$ & 2.7 \\
$50 \_55$ & $63 \%$ & $44 \%$ \\
$>120$ & $.25 \%$ & $0.1 \%$ \\
\hline
\end{tabular}

Table AA-1: Jet ET distribution for Herwig and Isajet. Herwig generates a higher fraction of higher as well as lower $\mathrm{E}_{\mathrm{T}}$ jets.

The ET distribution is all in all similar. HERWIG clearly has more lower as well as higher $\mathrm{E}_{\mathrm{T}}$ jets. We compared various Level 1 triggers which required various $\mathrm{E}_{\mathrm{T}}$ thresholds and various number of trigger towers. The following was observed:

1) The total ET triggers fired the same percentage of the time within $4 \%$. 2) The triggers that asked for 1 or 2 trigger towers above $3,4,5$ or $6 \mathrm{GeV}$ fired the same percentage of the time within $1 \%$.

3) If 3 or 4 towers are required, then HERWIG was systematically more efficient especially at the $6 \mathrm{GeV}$ threshold where it was $5 \%$ more efficient for 3 towers and $10 \%$ for 4 towers. This difference seems to decrease as the ET of the jets in the sample rises. This would tend to indicate that HERWIG jets deposit their energy in less space or perhaps that the core is more concentrated at least for jets up to $40 \mathrm{GeV}$.

\section{Sensitivity to Noise.}

Noise is the presence of a signal (usually with a constant mean and width) in the absence of particles in the detector. We now would like to compare the trigger efficiencies based on varying the Level 1 simulator with noise and without. We should note that at the time the study was done, the Level 1 simulator had much larger noise than it was programmed to have after the first cosmic ray commissioning run. The widths of the noise were approximately $250 \mathrm{MeV}$ in the electromagnetic trigger towers and 700-900 MeV in the hadronic portion. A 2.5 sigma zero suppression was 
applied to every tower. We looked at trigger efficiencies for various types of physics processes.

\section{$50 \mathrm{GeV}$ Single Electrons:}

For $50 \mathrm{GeV}$ electrons the efficiencies are virtually identical with and without noise if 1 tower above a threshold is required. At the $3 \mathrm{GeV}$ threshold, the efficiency doubles if $\mathrm{N}=2,3$ or 4 towers above $3 \mathrm{GeV}$ are required and the noise is turned on in the simulation. But these efficiencies are low as electrons tend to enter one trigger tower only.

\section{$50 \mathrm{GeV}$ Single Pions:}

For $50 \mathrm{GeV}$ pions, we only see an effect when $\mathrm{N}=3$. Without noise, the pion is very inefficient at these thresholds. But with noise, the triggering rates go up by a factor of about 5. We theorize that the pions spread out enough energy to allow noise effects to 'build' upon the lower towers and manage to trigger.

\section{0 jets in a variety of ET samples:}

Table AA-3 shows the multiplicative increase in triggering efficiency when the noise is turned on in the simulation. We see two trends. First, the lower ET bins have larger increases in trigger efficiency. Secondly, the lower trigger tower thresholds are more sensitive to the noise. The $5 \mathrm{GeV}$ threshold is relatively insensitive to noise while the higher thresholds are insensitive. We expect that the real determining factor is the ratio of the noise level to the trigger tower threshold. 


\begin{tabular}{|l|l|l|l|l|l|l|l|}
\hline$\#$ of TT & Et Cut & $2 \_5$ & $5 \_10$ & $10 \_20$ & $20 \_40$ & $40 \_80$ & \\
\hline 1 & 3 & 1.5 & 1.6 & 1.1 & 1.09 & 1.0 & \\
\hline 1 & 5 & 1.0 & 1.0 & 1.04 & 1.0 & 1.0 & \\
\hline 2 & 3 & 3.0 & 2.5 & 1.5 & 1.09 & 1.0 & \\
\hline 2 & 5 & & 1.0 & 1.1 & 1.1 & 1.03 & \\
\hline 3 & 3 & $.008 / 0$. & 1.5 & 2.0 & 1.2 & 1.03 & \\
\hline 3 & 5 & & & 1.0 & 1.03 & 1.05 & \\
\hline 4 & 3 & & $.007 / 0$ & 2.3 & 1.3 & 1.05 & \\
\hline 4 & 5 & & & & 1.3 & 1.1 & \\
\hline
\end{tabular}

Table AA-2: Ratio of Level 1 Trigger Tower efficiency with noise to without.

\section{Sensitivity to Energy Resolution}

We also look at the effect of calorimeter resolution on the Level 1 trigger efficiencies. We compare an old set of events in which smearing was $30 \% / \sqrt{E}$ for electrons and 60\%/VE to new numbers that are $16 \% / \sqrt{E}$ for electrons and $40 \% / \sqrt{E}$ for pions. Table AA-3 shows the ratio , $R$, of the rate with the old smearing with respect to that with the new smearing. This ratio is significantly greater than one only for the $3 \mathrm{GeV}$ thresholds and the lowest $\mathrm{E}_{\mathrm{T}}$ bins. But it is here that much of the rate comes from. Thus it is important to know the Level 1 energy resolution. We see that the high $\mathrm{ET}_{\mathrm{T}}$ event efficiencies are relatively unchanged at all triggers. But the 2_5 and 5_10 are widely different. Since the QCD jet ET spectrum falls as $E_{T}$ to a power, these small ET bins can contribute sizable amounts of rate which will decrease the signal to noise of the events that pass through the Level 1. 


\begin{tabular}{|c|c|c|c|c|c|c|}
\hline \# of towers & ET Cut & 2.5 & $5 \_10$ & $10 \_20$ & $20 \_40$ & $40 \_80$ \\
\hline 1 & 3 & 7 & 2.2 & 1.15 & 1.02 & \\
\hline 1 & 5 & 3 & 1.3 & & 1.01 & \\
\hline 1 & 7 & & & 1.04 & 1.02 & \\
\hline 1 & 9 & & & 1.25 & 1.02 & \\
\hline 2 & 3 & 2 & 3.7 & 1.5 & 1.08 & \\
\hline 2 & 5 & & & & 1.06 & \\
\hline 2 & 7 & & & & 1.09 & \\
\hline 2 & 9 & & & & & \\
\hline 3 & 3 & infinite & 2.0 & 1.7 & 1.18 & \\
\hline 3 & 5 & & & 3.0 & 1.11 & \\
\hline 3 & 7 & & & & & \\
\hline 3 & 9 & & & & & \\
\hline 4 & 3 & & & 3.0 & 1.38 & \\
\hline 4 & 5 & & & & 1.5 & 1.07 \\
\hline 4 & 7 & & & & & 1.07 \\
\hline 4 & 9 & & & & & \\
\hline
\end{tabular}

Table AA-3: Ratio of Trigger Efficiency with old smearing compared to new smearing: 


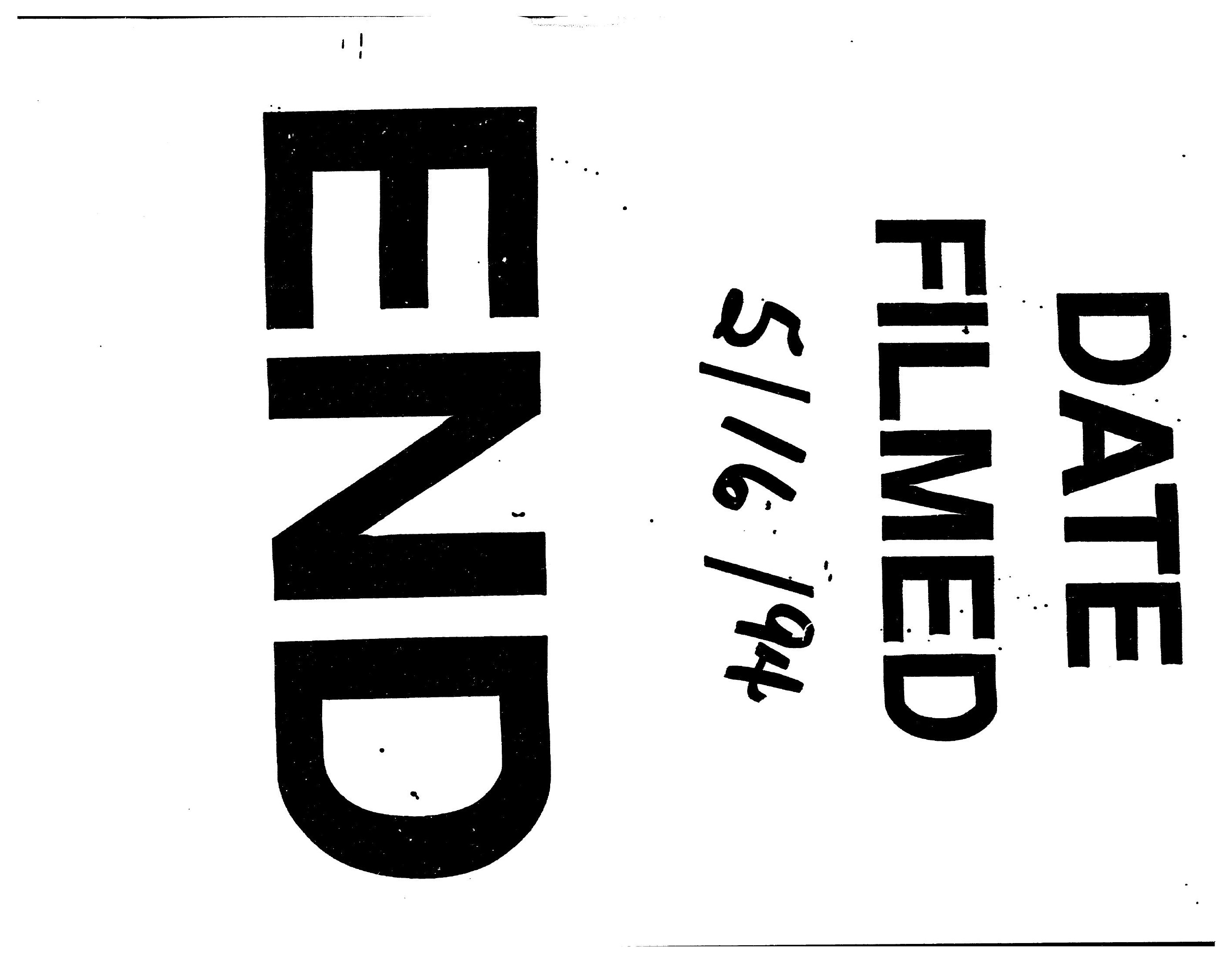


